\title{
RISKY RESIDENCES: AN EXPLORATORY STUDY OF SEXUAL VIOLENCE IN UNIVERSITY HALLS OF RESIDENCE
}

\author{
By \\ Samantha Maree Keene
}

\begin{abstract}
A thesis
submitted to the Victoria University of Wellington in fulfilment of the requirements for the degree of Master of Arts in Criminology
\end{abstract}

Social and Cultural Studies

Victoria University of Wellington

2015 


\begin{abstract}
Sexual violence within university populations is a well-known problem, however relatively little is known about the experience of sexual violence among New Zealand university students. There is even less known about women's experiences of sexual violence occurring in university halls of residence. This thesis addresses this gap in knowledge and understanding. Influenced by feminist perspectives and adopting a qualitative framework, this research employed semi-structured face-to-face interviews with four victims/survivors of sexual violence in university halls, and six key informants who work with students living in student accommodation or at the wider university. This study found that sexual violence occurring in halls had devastating impacts for women, affecting their personal, emotional, social and academic worlds. Further, this study also found that women were unlikely to disclose through formal channels such as the Police, but disclosure to informal supports was common. As well as this, data suggested that responding to sexual violence in this context is complex, as the needs of both alleged victims and alleged perpetrators must be carefully balanced. This complexity has meant that current responses to sexual violence are in many ways reactive rather than proactive and need to be comprehensively developed to respond to the unique challenges provided within the university environment. The findings from this study support the development of robust sexual violence response processes and the widespread delivery of education about sexual violence among university students, as well as for those charged with managing students' welfare.
\end{abstract}




\section{Acknowledgements}

This thesis has been a real labour of love, and there are so many people I am indebted to for their love and support throughout its completion. Writing this acknowledgements section is a reminder of how lucky I am.

First and foremost, I would like to thank all of the participants in this research that helped make it a reality. To the women who disclosed their personal experiences, as difficult as it was, and to the key informants who supplied invaluable commentary on such a tough issue - thank you. I am forever grateful for your willingness to share your stories with me and I hope you are happy with the final product.

Mum, without your support I would not be where I am now. Thank you for your unconditional love, guidance, and all the help you've given me during my 'extended stay' at university. Aunty Rach, thank you for being a pillar of support through all the rough times and always being there when I've needed someone to talk to. Grandma, thanks for your ongoing and interesting commentary on my thesis topic - it has surprisingly given me more motivation to complete it when I've felt like giving up. Ashleigh, thank you for your support - I hope that someday you'll properly understand what degree I enrolled in and what I've spent the last 18 months doing! Reece, thank you for being someone I know will always be there, no matter what happens. Without you all, who knows what this thesis would have ended up looking like.

Poppy, you mean more to me than you'll ever know, thank you so much for all your love, support and late night debriefs when things have been too much. Lucas, your cheeky grin has cheered me up more times than I can count when this thesis has been overwhelming. Toby, you have been a huge source of excitement this year, and I can't wait to meet you soon!

I owe a big thank you to my extensive network of friends that have been there for me in so many ways. Philip Bateman, Sam Calvert, Toni Carr, Jamie Dwyer, Kirsten Gibson, Cara Gledhill, Richard Greathead, Hendrix Joass, Ryan Kane, Rhys Katon, Emma Kitson, Jane Murphy, Dave Roberts, Woody Robinson, Matt Rolfe, Aimee Stephens, and Jared Walton. You guys are the best and I have been 
so lucky to have your support and friendship during my 'professional student' days.

I was also lucky enough to also have a fabulous team of editors who helped make this thesis presentable. Sam Calvert, Cara Gledhill, Alexis Harris, Syreeta Heke, Imogen Holmstead-Scott and Lauren Nyhan - you are absolute stars!

I would like to extend a big thank you to all the staff on the $9^{\text {th }}$ and $11^{\text {th }}$ floors that have provided me with advice and support throughout. I would also like to thank Elaine Mossman for her help and support in the initial stages of thesis development.

I owe a huge thank you to the New Zealand Family Violence Clearinghouse for helping making this thesis a reality by providing financial support.

Lastly, I am forever indebted to my amazing supervisor, Jan Jordan. Jan, without your support and guidance, I know this thesis would not even be started. Thank you for tolerating my self-doubt and giving me the confidence kick I needed to start it, and unbelievably, complete it. Thank you also for helping me acknowledge the times when I need to lessen the load and put myself first, as hard as it was to hear! Working with you as my mentor for the past few years has meant so much to me. I cannot thank you enough for everything that you have done and I look forward to working with you in the future. 


\section{Table of Contents}

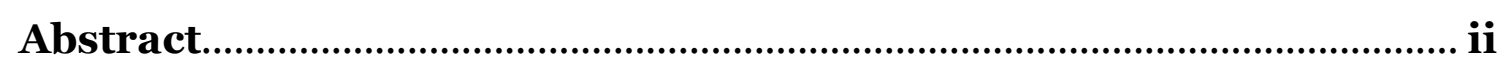

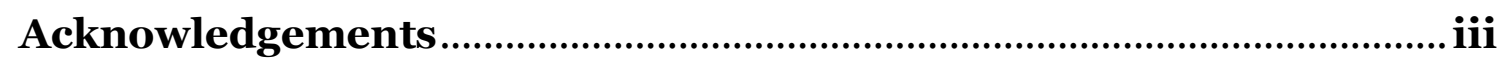

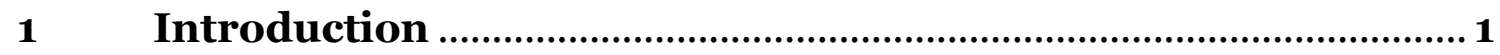

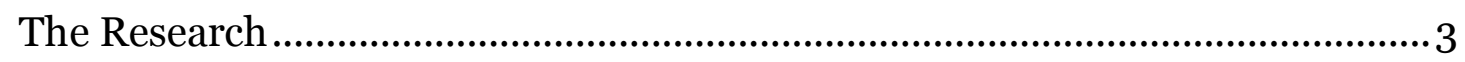

Thesis Overview ................................................................................................. 4

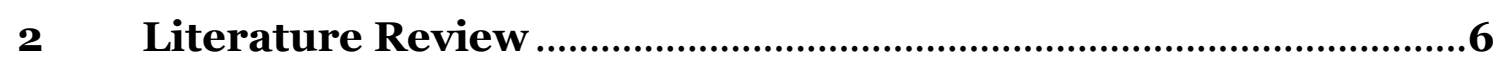

Background to the Review ............................................................................... 7

Students and Sexual Violence Prevalence …………………………………....

Sexual Violence in University Halls .......................................................................11

Students as a Population 'At Risk' .................................................................. 12

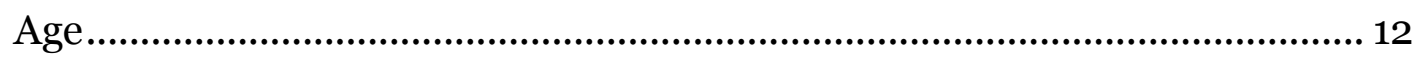

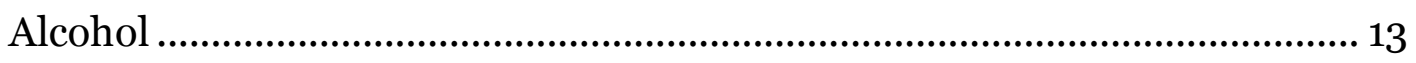

Revictimisation ....................................................................................... 14

Rape-supportive beliefs ............................................................................. 15

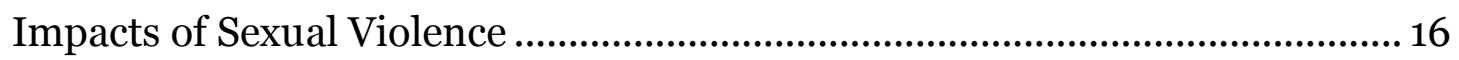

Disclosure of Sexual Violence .......................................................................... 17

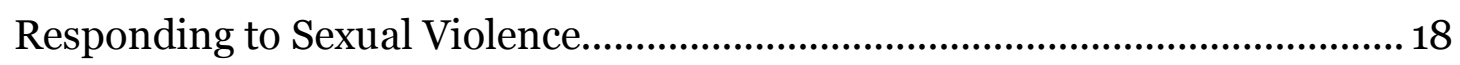

Preventing Sexual Violence............................................................................... 19

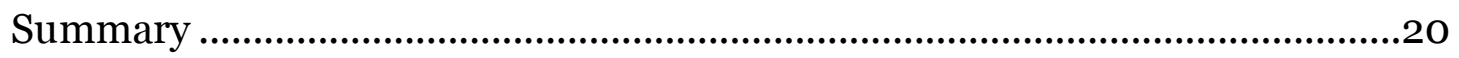

3 Methodological Framework ……………………………………..21

Theoretical Approach ....................................................................................22

Methodological Approach ..............................................................................23

Research Methods ........................................................................................24

Initial Research Design ........................................................................24

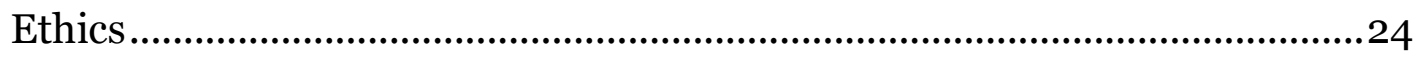

Participants, Data Collection and Analysis........................................................25

Participants ............................................................................................25

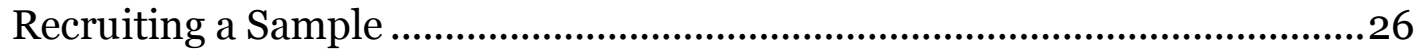

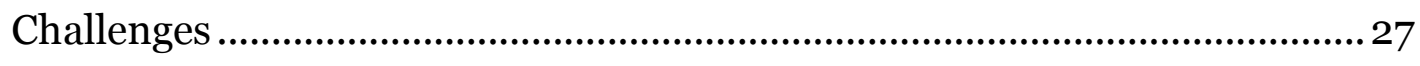

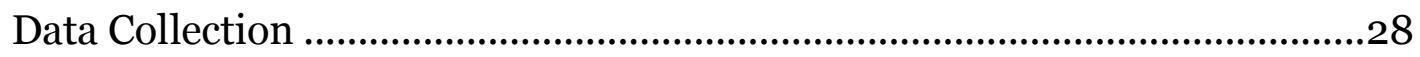

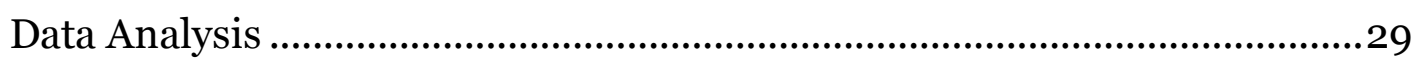

Presentation of Data....................................................................................... 
Limitations 30

Personal Challenges and Transformations

4 Expectations, Risks and Realities: A Background to the Findings 33

Life in a University Hall ................................................................................34

The Influence of American Fraternity Imagery? ...................................................35

Risk in Halls of Residence.................................................................................36

Mixed-gender Living and Public Space ..............................................................36

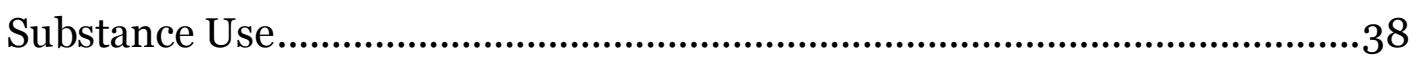

Hook-up Culture.....................................................................................................

Sexual Pressure and Navigating Consent ..........................................................40

Realities of Living in the Mix .........................................................................42

Environment and Public Space .........................................................................42

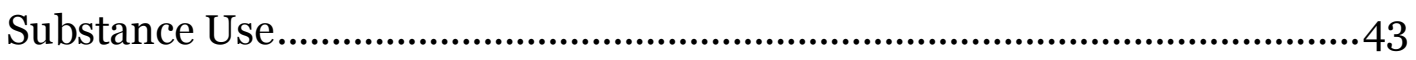

Participants' Experiences and Knowledge of Sexual Violence in Halls.............44

Sexual Violence Experiences and Reflections .....................................................44

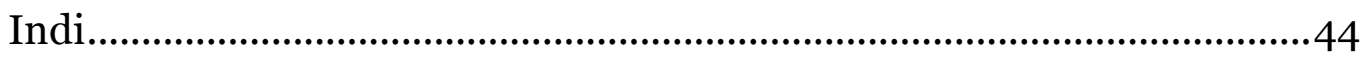

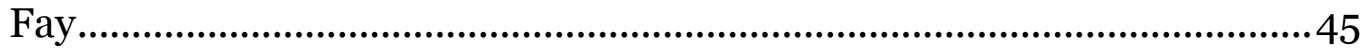

Bianca …….........................................................................................

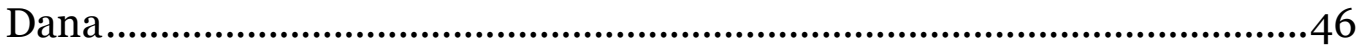

The Framework for the Following Chapters .....................................................48

\section{Sexual Violence and its Consequences: The Impacts of Experiencing Sexual Violence .......................................................................... 49}

Psychological Impacts ......................................................................................50

Affecting 'the self' ...............................................................................................50

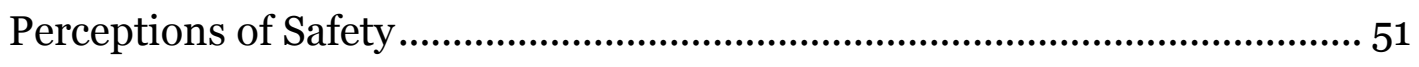

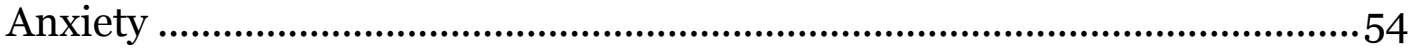

Alcohol and Other Substance Use................................................................... 55

Difficulties Sleeping …………………….........................................................56

Flashbacks and Panic Attacks .........................................................................56

Social Impacts ..............................................................................................5

Difficulties in Interpersonal Relationships ..................................................58 


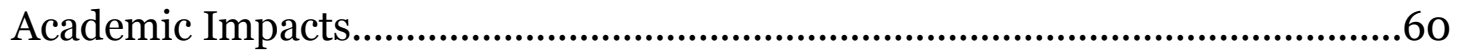

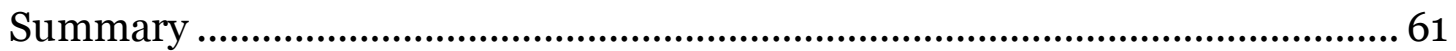

6 Disclosing Sexual Victimisation and Seeking Help: In Whom do University Women Confide?...................................................................63

Formal Disclosures .............................................................................................64

Underreporting of Sexual Violence to the Police ................................................64

Participants' Experiences with Disclosure to the Police ..................................65

Disclosures to Others............................................................................................66

Hall Management ..................................................................................................67

Residential Assistants .......................................................................................69

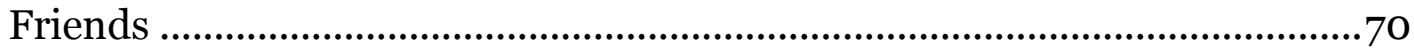

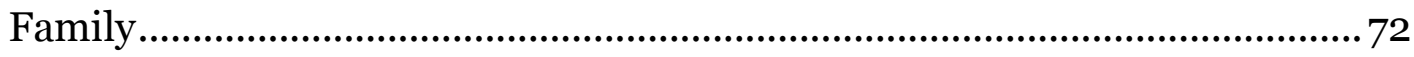

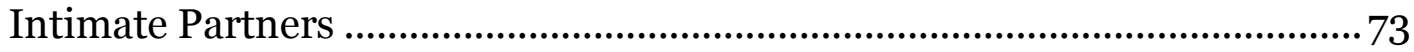

Help-Seeking Utilisation ..................................................................................... 74

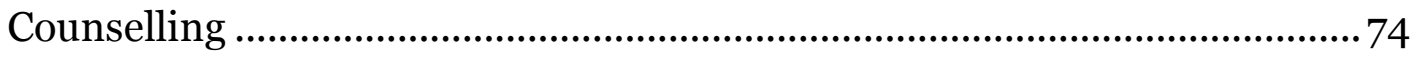

Summary …….................................................................................................... 77

$7 \quad$ Dealing with Sexual Violence ……………………………………....... 79

Responding to Sexual Violence Within University Populations........................ 80

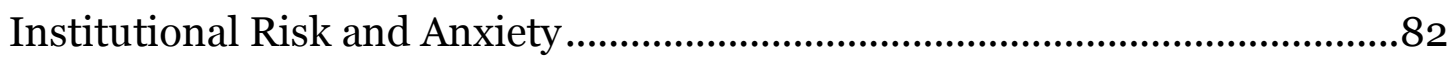

Current Responses and Avenues for Change.....................................................84

Perspectives of Key Informants......................................................................84

Education and Awareness-Raising................................................................ 87

Coordinated Response to Sexual Violence …………………………….......89

Restorative Justice for Halls of Residence …………………….....................90

Perspectives of Victims/Survivors ................................................................... 91

Validation and Agency.................................................................................... 91

Confidence and Trust in Management..........................................................92

Education and Information .........................................................................93

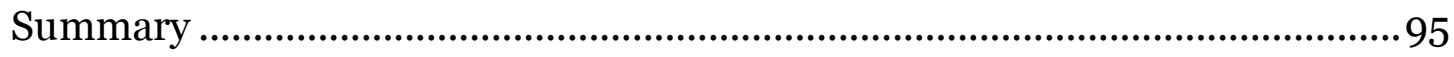

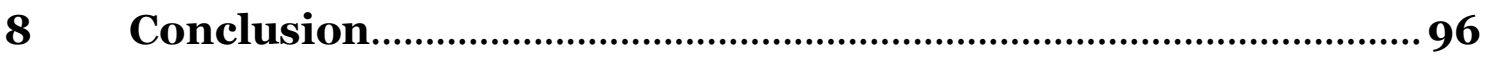

Identifying the Scope of the Issue .....................................................................97

Living in a Hall of Residence: Risky Living? ......................................................97 
The Consequences of Sexual Violence .................................................................99

Sexual Victimisation Disclosure: Speak Out or Stay Silent? ............................ 101

Dealing With Sexual Violence: How to Respond? .............................................103

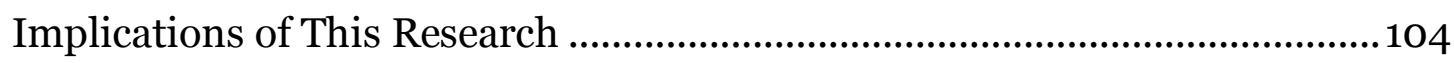

Recommendations for Best Practice in Responding to Sexual Violence......... 105

Recommendations for Best Practice in Preventing Sexual Violence............... 107

Looking to the Future........................................................................................108

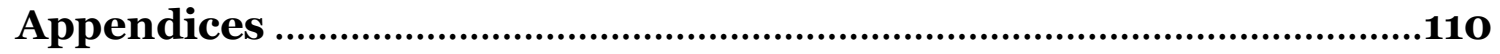

Appendix A: Ethical Approval ..................................................................111

Appendix B: Recruitment Flyer ........................................................................114

Appendix C: Key Informant Information Sheet .................................................115

Appendix D: Key Informant Consent Form ……………......................................117

Appendix E: Key Informant Interview Guide....................................................... 118

Appendix F: Participant Information Sheet .........................................................119

Appendix G: Participant Consent Form................................................................121

Appendix H: Participant Interview Guide ......................................................... 122

Appendix I: Participant Support Services Sheet ................................................ 123

References ........................................................................................ 124 


\section{Introduction: Setting the Scene}

When female students embark on a college career, they bear the unwarranted cost of the threat and reality of being raped, sexually assaulted, harassed, and stalked. For many years, this cost remained hidden from public view. Victims were left to suffer in silence; their voices were not heard and their pains were ignored (Fisher, Daigle \& Cullen, 2009: 2).

Violence against women is a global concern and a common experience for many women, with New Zealand estimates suggesting that as many as one in three experience such violence in their lifetime (Fanslow \& Robinson, 2004; Mayhew \& Reilly, 2007). Alarmingly, particular groups of women experience sexual violence at elevated rates, with one of those groups being female university students (Gross, Winslett, Roberts \& Gohm, 2006; Fisher et al., 2009; Walsh, Banyard, Moynihan, Ward \& Cohn, 2010). A substantive body of international research documents students' experiences of sexual victimisation, with recognition of the issue being shown in the United States (US), United Kingdom $^{1}$ and Australia ${ }^{2}$, however little New Zealand data exists on the issue. There is even less data on students' experiences of sexual violence in New Zealand university halls of residence. This thesis addresses this literature gap and provides the first insight into sexual violence in New Zealand halls, specifically at Victoria University, by analysing interviews provided from both women who have experienced sexual violence, as well as key informants who work with student populations.

\footnotetext{
1 Recent research by the National Union of Students (2010) has drawn attention to the pervasiveness of sexual victimisation in the United Kingdom and is sparking calls for more adequate responses.

2 Research by Boursnell, Lee and Chung (2008) surveyed student attitudes toward sexual violence and research by Mikhailovich and Colbran (1999) explores sexual violence responses at an Australian university. This work acknowledges the pervasiveness of the issue.
} 
The lack of information about sexual violence among students contributes to a lack of effective responses and prevention efforts to ensure students' safety. My research documents the unique environmental and cultural factors that may contribute to elevated sexual violence among student populations, as well as documenting four women's experiences of sexual victimisation in their university halls. Further, it discusses the debilitating impacts that sexual victimisation can, and does, have on female students, as evidenced by interviews with the women in this research. This thesis also highlights women's experiences with disclosing their victimisation, and acknowledges in particular the difficulties and complexities involved in disclosing sexual victimisation to the police, hall management, and other less formal supports such as friends, family and intimates. Lastly, this thesis documents the current response and prevention efforts implemented in Victoria University of Wellington halls of residence. It identifies how institutional anxieties about reputational impact may have impeded ongoing and dedicated efforts to protect students' welfare and wellbeing, and discusses recommendations for the future to enhance students' safety and reduce the risk of sexual victimisation that women face in their university residences.

It is critical to clarify the definition of sexual violence used for this research. Globally pervasive issues such as sexual violence are notoriously difficult to define, and there is a lack of agreement on a universal definition (Bourke, 2007). Scholarly disagreement has resulted in the use of differing terminology throughout research, which causes inherent difficulties in comparing data sources. Whilst a full contribution to the definitional debate cannot be accommodated here, this thesis adopts the definition of sexual violence outlined by the World Health Organisation (Jewkes, Sen \& Garcia-Moreno, 2002: 149):

Any sexual act, attempt to obtain a sexual act, unwanted sexual comments or advances, or acts to traffic, or otherwise directed, against a person's sexuality using coercion, by any person, regardless of their relationship to the victim, in any setting, including but not limited to home and work.

The reason for using this definition is twofold. Firstly, it is wide, inclusive and makes specific mention of the relationship between the victim and perpetrator. Secondly, it also identifies that sexual violence can take place in any setting. As 
this thesis will show, both of these parameters are important considerations when analysing sexual violence among student populations. I would also like to acknowledge the importance of Liz Kelly's (1988) work on surviving sexual violence, and situate the definition of sexual violence used here within a lens that views all sexual violence as a form

It is also important to clarify the terminology used in this thesis when discussing women's experiences of sexual violence. There is substantive debate about the usage of the terms 'victim' or 'survivor' when discussing women's experiences of sexual violence. This debate stems from awareness that the term 'victim' can be a reinforcement of women's passivity, whilst 'survivor' equates with "active resistance and sense of agency" (Jordan, 2001: 9). However, labelling women as either victims or survivors disregards the possibility that the journey from victim to survivor can be a difficult one, with both terms likely to be relevant to different aspects of women's experiences at varying times of their lives (Jordan, 2013). To acknowledge that women can be both victims and survivors, I use the term 'victim/survivor' in this thesis when referring to women's experiences of sexual violence.

\section{The Research}

The objective of this research is to explore women's experiences of sexual violence in New Zealand university halls. This thesis draws upon female victims/survivors' experiences in Victoria University of Wellington residences, as well as professional perspectives on sexual violence among students provided by key informants. To achieve this, the following questions were adopted to guide the research process:

1. What circumstances existed that may, or may not have, contributed to sexual violence in their university hall?

2. Were there any ways in which participants felt that their residence could have done more to keep them safe?

3. In the event of a disclosure, how did participants feel they were treated by friends, family or institutions?

4. What do participants feel are appropriate institutional responses to sexual victimisation in halls of residence? To what extent do current university and hall responses align with victims/survivors' views? 
The impetus for this research comes from an interest in the area of student victimisation in halls, stemming from an Honours-level research project. As well as this, my own experience living in a hall of residence, and my personal reflections on the things I saw and noticed in my hall, contributed to the decision to research this area. Further, it also comes from a place of uncertainty about the levels of sexual violence in New Zealand universities and their affiliated halls, largely due to an absence of literature on the issue. Therefore, this research is largely exploratory in nature and provides the first qualitative insight into sexual victimisation experiences among students in New Zealand halls.

\section{Thesis Overview}

This thesis draws on interviews from both victims/survivors of sexual violence in halls, and key informants working with students to provide a starting point for discussions on student victimisation in New Zealand. Further, it is hoped that this research will contribute to greater acknowledgement by universities and hall management of the risks women face in university halls, as well as the development and implementation of sexual violence response protocols and prevention programmes to better protect students. The structure of this thesis is largely determined by the findings and analysis of the data collected.

Having highlighted the New Zealand context for this research and outlined the research aims, Chapter Two reviews relevant literature on the sexual victimisation of university students, providing a framework to use in the interpretation of research findings. Chapter Three discusses the methodological approach for this research, outlining the research methods used as well the limitations and personal challenges faced throughout. Chapter Four sets the scene for later chapters by documenting the risk factors that may contribute to sexual violence in universities and their affiliated halls for female students. Further, it shows how those risks became reality for the women interviewed for this research. Chapter Five outlines the impacts and consequences that experiencing sexual violence had on participants, which affected their psychological, social and academic worlds. Chapter Six documents women's experiences with disclosing sexual violence to both formal and informal supports. Chapter Seven explores current responses and prevention initiatives 
at Victoria University, and examines the role that institutional anxiety plays in impeding developed and consistent responses and prevention programmes. Lastly, Chapter Eight concludes this thesis, and discusses recommendations for the future regarding sexual violence response protocols and prevention initiatives to enhance students' safety and wellbeing. 


\section{Literature Review}

...College campuses are social domains conducive to students' sexual victimization, including rape. There are times when coeds walking alone at night are sexually assaulted by a stranger. But beyond these disturbing crimes, the risk of female students' victimization is ingrained in the very fabric of normal college life (Fisher et al., 2009: 2).

This chapter provides a review of the literature on sexual violence among university students, particularly students in university halls. The review begins by identifying sexual violence within a wider social context, viewing it as an embedded part of our social fabric. This chapter then situates university students within this as an at-risk population. An analysis of literature on student victimisation is provided which identifies a number of gaps within the literature base, as well as gaps in official responses. Given the lack of research about sexual violence among university students in New Zealand, this chapter relies heavily on research from the United States. However, where possible, literature from outside the United States or from New Zealand is used. Further, this chapter only investigates female students' experiences of sexual violence as that is the object of this research. 3

3 It should be noted that men can be victims of sexual violence. For research on men's experiences of sexual violence in a university context, see Banyard, Ward, Cohn, Moorhead \& Walsh, 2007). 


\section{Background to the Review}

Violence against women and girls is a global concern, however quantifying violence against women is a complex task, as measuring the incidence of such a personal crime is difficult. In fact, Bourke (2007) argues that statistics on sexual violence prevalence cannot exist, as sexual violence "eludes statistical notation" (p. 15). New Zealand research reports that 29 percent of women have experienced sexual violence in their lifetime (Mayhew \& Reilly, 2007), however the complexities involved in acknowledging, disclosing and reporting sexual violence mean that this may be an underestimate of its true incidence (Kelly, 1988; Wykes \& Welch, 2009).

Sexual violence is not a new phenomenon, rather the "history of rape is the history of women" (Jordan, 2001: 21). Depictions of rape have permeated historical writings, and rape legislation has traditionally been framed from a male perspective (Bourke, 2007; Jordan, 2011), overshadowing the views of victims/survivors and making rape "the act of having sex with a woman who does not 'belong' to the perpetrator" (Bourke, 2007: 9). However, the very use of rape to acquire a woman as property is cemented in historical accounts of 'bride capture' that existed until the $15^{\text {th }}$ century (Brownmiller, 1975). These historical links between sexual violence and marriage not only undermine the seriousness of sexual violation (Jordan, 2001), but also illustrate the legacy of patriarchy in society.

Feminist activism from the 1960 s onwards drew attention to the scale of rape, and the tremendous work done by feminist activists is credited for the shifts in societal understandings of rape today (Gavey, 2005). Second-wave feminists (e.g. Brownmiller, 1975; Russell, 1975; Griffin, 1977) challenged the patriarchal structures that condoned violence against women and brought women's experiences of sexual violence into the public arena for the first time (Fisher et al., 2009). Further, Liz Kelly's (1988) research on surviving sexual violence established sexual violence as occurring on a continuum, thus acknowledging women's experiences of gendered coercion and control, and highlighted the extent that such experiences were mirrored among women. Ongoing protest and 
action by feminists has encouraged developments in rape legislation and has been a significant contributor to increasing gender equality.

It seems unfathomable then, in light of these feminist successes, that sexual violence continues to be a problem in a developed country such as New Zealand, the first to allow women the vote (Smith, 2012) and has women holding powerful positions in society. Legislative change has been slow, with men remaining exempt from prosecution for raping their wives until 1985 (Else, 2012). Until 2013, Ministerial responsibility for sexual violence sat across a number of portfolios, contributing to problems with accountability and multiagency collaboration. Whilst the government appointed a Minister responsible for sexual violence in 2013, ${ }^{4}$ results from the September 2014 election now show no Member of Parliament holds Ministerial responsibility for the portfolio (Department of Prime Minister and Cabinet, 2014). There remains resistance to consistent funding for sexual abuse support and crisis services, and services have had to close in recent years due to a lack of funding (Gousmett, 2013; Radio New Zealand, 2014). Further, women continue to experience sexual violence at far higher rates than men (Mayhew \& Reilly, 2007) and many victims are still silenced (Jordan, 2012). These realities highlight how sexual violence remains an embedded part of New Zealand's social fabric.

Recent public outrage and criticism of criminal justice responses to sexual violence has brought sexual violence to the fore in New Zealand public consciousness. The Roast Busters scandal 5 and the response by the New Zealand Police and Government to Tania Billingsley's sexual assault claim against Malaysian diplomat Muhammad Rizalman6 have brought sexual violence to national attention. Further, one million dollars has recently been awarded to researchers at Victoria University of Wellington to investigate New Zealand's

\footnotetext{
4 Paula Bennett took Ministerial leadership of the sexual violence sector in February 2013 (Bennett, 2013).

5 The Roast Busters were a group of young New Zealand men who were allegedly involved in group sex with underage and intoxicated girls. The case sparked significant social uproar both at the actions of the men involved, as well as the New Zealand Police investigation, which required an inquiry in their handling of the case. On October 29, 2014, Police announced that no charges would be laid due to a lack of evidence (Steward \& Dennett, 2014).

${ }^{6}$ Muhammad Rizalman was arrested and charged with burglary and assault with intent to rape, but was subsequently allowed to invoke diplomatic immunity. Ministers were aware of the case, but had not investigated it further. An inquiry into the handling of the case by the Ministry of Foreign Affairs and Trade has been launched (Knight, 2014).
} 
rape culture.7 Although New Zealand arguably still has a rape culture, as demonstrated by the aforementioned events, feminist successes and changes in legislation have not "seduced women into submission" (Pearson, 2003: 63); rather, sexual violence no longer exists in New Zealand "without fierce contestation on multiple fronts" (Gavey, 2005: 17).

\section{Students and Sexual Violence Prevalence}

Although prevalence rates of sexual violence are incredibly high, there are groups within society who experience it at higher rates than others (Daigle, 2012). One such group is university students (Mayhew \& Reilly, 2007; Fisher et al., 2009). Students' experiences of sexual violence have received significant recent attention to date in the United States (US), but it was not until the 1980 s that sexual violence among university students was recognised as a pervasive issue. Early research (e.g. Kilpatrick \& Kanin, 1957; Kanin and Parcell, 1977; Koss and Oros, 1982) highlighted the existence of sexual violence among university students, but were not as influential as Koss, Gidycz and Wisniewski's (1987) national study of campus sexual violence. Koss et al. (1987) surveyed 6159 students at 32 higher education institutions in the US and produced some staggering findings. Of the women reporting victimisation since age 14, over 53 percent had experienced some form of sexual violence, nearly 12 percent had experienced sexual coercion, just over 12 percent had experienced an attempted rape and over 15 percent had been raped. Of women reporting sexual violence in the previous year, over 46 percent had experienced some form of sexual victimisation and 6.5 percent of the sample had been raped.

Mary Koss's groundbreaking work established sexual violence on campus as an endemic issue, and investigation into sexual violence on campus continues to be a topic of popular interest, especially in the United States (Fisher et al., 2009). Recent research investigating the prevalence of sexual violence among university students repeatedly suggests that as many as one in four students will experience sexual violence during their time at university (Gross et al., 2006; Kilpatrick, Resnick, Ruggiero, Conoscenti \& McCauley, 2007; Fisher et al.,

\footnotetext{
7 Associate Professor Jan Jordan has been granted $\$ 610,000$ for a research project investigating barriers to rape reform, and Associate Professor Elisabeth McDonald has been awarded $\$ 540,000$ to investigate rape myths as barriers to fair trial practices (Victoria University of Wellington, 2014).
} 
2009; Krebs, Lindquist, Warner, Fisher \& Martin, 2009; Walsh et al., 2010). In fact, some estimates even suggest this number is higher, with one study reporting that 38 percent of their sample reported one or more episodes of sexual victimisation (Nasta et al., 2005). These estimates suggest that not only is sexual violence on campus a popular area of research, rather they highlight how sexual violence among university students continues to be an endemic issue, nearly thirty years after it was first identified as a problem.

Although there is heightened interest in researching sexual violence among students in the United States, there is a very small literature base on the area in New Zealand, despite the 2006 New Zealand Crime and Safety Survey reporting that students were a group at high risk of sexual victimisation (Mayhew \& Reilly, 2007). The first study of sexual violence among New Zealand university students was by Nicola Gavey in 1991. Gavey explored sexual victimisation among first-year psychology students at Auckland University, finding that 52 percent of participants reported some form of sexual victimisation over the past three years. Although not specific to sexually violent experiences at university, it is likely that a number of participants had experienced sexual violence during that time, which could have occurred on campus or in halls of residence. Nevertheless, Gavey's work was the first focused study of sexual violence among New Zealand university students.

Later research into sexual violence among students took a decade; however there are a number of public health articles that have explored sexual victimisation, often as part of a wider focus on alcohol-related harm. Research at Otago University continually reports high rates of unwanted sexual advances and sexual assaults over four week periods (Langley, Kypri \& Stephenson, 2003; Cashell-Smith, Connor \& Kypri, 2007). Further, 2010 data drawn from six university campuses reported that 21 percent of women had experienced an unwanted sexual advance, and 0.5 percent had experienced sexual assault or date rape, in the preceding month (Connor, Gray \& Kypri, 2010). Most recently, a national study of nearly 300 students investigated risky drinking and risky sex among New Zealand students. This sample reported that in the last month, 15 percent of women had experienced an unwanted sexual advance and 0.7 percent had been a victim of sexual assault or date rape (Connor, Psutka, Cousins, Gray 
and Kypri, 2013). The continually high prevalence rates in these New Zealand statistics highlight the significance of the issue on New Zealand university campuses and provide a platform for future research.

\section{Sexual Violence in University Halls}

Sexual victimisation is a broad area, and some scholars have narrowed the scope of their studies, such as a focus on how it may be linked to alcohol consumption (Abbey, McAuslan \& Ross, 1993; Abbey, 2002; Abbey, Clinton-Sherrod, McAuslan, Zawacki \& Buck, 2003). However, while there has been little dedicated research exploring the unique environments within halls of residence, many studies do use samples from halls or dormitories for convenience. This lack of research is surprising, as the living arrangements provided by student housing have been recognised elsewhere to "pose proximity-to-offender situations and, thus, increase the risk of victimization" (Fisher \& Wilkes, 2003: $529)$.

In the early 1990s, Canadian researchers investigated experiences of unwanted sexual attention in a university residence (Cairns \& Wright, 1993). The following year, an analysis of qualitative data from the survey was conducted which showed the commonness of sexual coercion in the residence, as students "told numerous stories of experiencing sexual coercion” (Cairns, 1994: 197). A few years later, a Master's thesis explored sexual harassment in the same university's halls of residence. In Borbridge's (1996) survey of 93 women living in the residence, just over 57 percent of participants had experienced unwanted attempts to kiss or fondle during their time there.

Recent research with university women suggests that almost 60 percent of completed rapes occurring 'on campus' happen in the victim's residence (Fisher, Cullen \& Turner, 2000). More recently, research by Walsh et al. (2010) has established that a substantive amount of unwanted sexual activity takes place in 'on-campus' residences such as university halls in the US. Such findings over the past two decades establish sexual violence in university halls as an issue of substantive concern.

The data available on sexual violence among students living in halls in New Zealand is minimal. However, Cashell-Smith et al.'s (2007) research on alcohol 
consumption and risky sexual behaviour provides useful data on the issue. In their sample of 1564 students, over 28 percent reported unwanted sexual advances in their residence hall in the last month, and just over 30 percent had engaged in risky sexual behaviour over the same period. Further, a recent Honours dissertation conducted an exploratory, qualitative study into the referral pathways for intimate partner violence and sexual violence among key informants at the University of Auckland. Qualitative data from a Head of Hall in this study suggested that although official reports were low, they perceived that there were a lot of incidents occurring which were unreported (Smith, 2013). These studies provide a starting point for future research and highlight sexual violence in halls of residence as an area of concern that warrants further academic attention.

\section{Students as a Population 'At Risk'}

The previously discussed prevalence data has identified the significance of sexual violence among student populations, and establishes them as a population that experience elevated rates of sexual violence. However, an understanding of the factors that contribute to elevated prevalence rates is necessary. This review will now explore a number of factors that contribute to elevated risk among students, with a particular focus on students living in halls of residence where possible.

\section{Age}

Research has identified the interplay between elevated risk and the age of both victims and perpetrators. Young women are frequently identified as being at the highest risk of experiencing sexual violence (Johnson, Ollus \& Nevala, 2007; Fisher et al., 2009; Flores, 2010; Cue Davis, 2011), especially between the ages of 15 and 24 (Mayhew \& Reilly, 2007; Katz, May, Sorenson \& DelTosta, 2010; Daigle, 2012). Further, young men are more likely to be involved in sexual violence perpetration during this same period (Flowers, 2009). This is an important consideration when researching sexual violence among students, as most students are in this age bracket (Garcia-Moreno, Mitchell \& Wellings., 2012). This also means that it is an important consideration when researching 
halls of residence, as most halls house first year students to ease the transition to independent living and university life (Zeller, 2010).

Schwartz and DeKeseredy (2007) argue that risk for sexual violence only exists during the first year of university study, however others suggest that the risk of experiencing sexual violence is at its peak during the first year, which Flack and colleagues (2008) term a "red zone", with elevated risk remaining an issue for second-year students (Fouts \& Knapp, 2001; Sampson, 2002). Krebs et al. (2009) further this, arguing that the risk of sexual violence decreases continually in correlation with the number of years spent at university, with the first year being the most dangerous. Although there is disagreement in this area, the heightened risk during the first-year cannot be ignored, and raises important questions about the role that university residences play in protecting such a vulnerable cohort of students.

\section{Alcohol}

The role of alcohol in sexual violence victimisation and perpetration has been the subject of significant research attention. Alcohol may be a contributing factor in some instances of sexual violence perpetration "because it allows perpetrators to focus on their immediate feelings of sexual desire and entitlement rather than on more distal cues such as the victim's suffering or their own sense of morality" (Abbey et al., 2003: 814). Further, alcohol use on the part of the victim may affect cognition and the ability to identify early signals or attempts at assault, as well as an inability to resist advances (Abbey et al., 2003; Crawford, O’Dougherty Wright \& Birchmeier, 2008).

Alcohol consumption among students is high, therefore a substantial body of research exists which documents the prevalence and impacts of elevated alcohol use, especially in relation to sexual violence (Abbey et al., 2003; Adams-Curtis \& Forbes, 2004; Cashell-Smith et al., 2007; Krebs et al., 2009; Connor et al., 2010; Smith, Magee \& Jones, 2012). Research with students in the US suggests that 50 percent of sexual assaults that occur within student populations are associated with alcohol consumption (Abbey et al., 1998; Fisher, Cullen \& Turner, 2000; Abbey, 2002). Although the links exist, this does not mean that the relationship is causal, as this ignores the personal, social and cultural 
dimensions that it occurs in. That said, the role that alcohol plays cannot be ignored either and incorporating such knowledge is important in the development and delivery of prevention efforts (Abbey, 2002; Lindquist et al., 2013).

Alcohol use at New Zealand universities is part of the university experience, with New Zealand's student drinking patterns described as a "culture of consumption" with "acculturation in heavy drinking habits coinciding with student arrivals at tertiary education institutes" (Towl, 2004: 7). Heavy drinking is a problem in university halls (Kypri, Paschall, Maclennan \& Langley, 2007) and students who live in a hall of residence are more than twice as likely as other university students to have two or more binge drinking 8 episodes during the week (Kypri, Paschall, Langley, Baxter Cashell-Smith \& Bourdeau, 2009). Therefore certain drinking locations, residential halls included, appear to promote or facilitate heavy alcohol consumption among students, which can be a contributing factor in students' elevated risk (Kypri et al., 2009). More New Zealand specific research regarding students' alcohol use, especially in halls, is warranted and would be useful in the context of prevention efforts in New Zealand.

\section{Revictimisation}

One of the most concerning findings in the sexual violence literature is that multiple or repeat victimisation is a common experience, and is one of the strongest predictors of future victimisation (Krebs et al., 2009; Daigle, 2012; Ministry of Women's Affairs, 2012). Further, there are disparities in the likelihood of experiencing revictimisation, and a small proportion of individuals experience a disproportionate share of all victimisation (Fisher et al., 2009). However, research in this area is still emerging, so there are few studies that have explored students' experiences with revictimisation (Krebs et al., 2009). Some research suggests that almost half of students reporting sexual assault in their lifetime report multiple victimisation experiences (Crawford et al., 2008). Data from the National College Women's Sexual Victimisation Study supports this, as over 61 percent of women who reported rape at university had

\footnotetext{
8 Binge drinking is defined as consuming five or more alcoholic drinks on the same occasion at least once in the past month (Durrant \& Thakker, 2003).
} 
experienced prior sexual victimisation (Fisher et al., 2009). Further, sexual revictimisation during time spent at university has been identified as an issue in a recent study, as "sexual revictimisation reported early in the first academic year predicted greater risk for victimization later that year" (Katz et al., 2010: 2121). These findings situate university students as a group at risk of experiencing revictimisation. Further, Katz et al.'s (2010) findings are evidence of the importance of ongoing research attention to the issue.

\section{Rape-supportive Beliefs}

Misconceptions about the realities of sexual violence contribute to its ongoing silence (Pearson, 2003). Rape myths encourage "prejudicial, stereotyped, or false beliefs about rape, rape victims and rapists" (Burt, 1991: 129). They also frame rape as a random, uncommon stranger rape scenario (Boskey, Harper \& Hilgenkamp, 2010), or blame the victim for their rape, disbelieve rape claims, claim women desire to be raped, exonerate the perpetrator or imply that only certain types of women get raped (Bohner, Friederike, Pina, Siebler \& Tendayi Viki, 2009).

The presence of rape-supportive beliefs among university students is an area of concern. As universities are microcosms of wider society (Mikhailovich \& Colbran, 1999), wider social stereotypes are transplanted to the university campus, with Ottens (2001: 6) noting that "by the time students reach college age, many are steeped in the conventional wisdom (myths) that justify violent behaviour against romantic partners.” Research has highlighted the presence of rape-supportive cultures and beliefs on university campuses (Argiero, Dyrdahl, Fernandez, Whitney \& Woodring, 2010) and research suggests a significant number of men would perpetrate sexually aggressive behaviour if they could be assured they would not be caught (Carr \& Van Deusen, 2004; Burgess, 2007). Unfortunately there is little information regarding rape myth acceptance among New Zealand university students, so an assessment of rape supportive cultures and beliefs is unable to be backed up by empirical research. However, recent cases involving New Zealand university students have highlighted the sexualised nature of university campuses, and the anecdotal presence of such views. ${ }^{9}$ Rape

9 There have been a number of recent cases where university students have been involved in sharing rape-supportive behaviours on social media. Rape jokes were shared on Victoria 
myth acceptance has been identified as an explanatory predictor in the actual perpetration of sexual violence (McMahon, 2010), so these findings encourage ongoing research attention, especially to incorporate in the development of prevention efforts.

\section{Impacts of Sexual Violence}

Sexual violence affects women differently, even if two women have experienced similar forms of violence, as the consequences of sexual victimisation are complex (Kelly, 1988). For example, women's experiences of sexual violence can, and often are, shaped by an individual's past and present experiences of assault, thus having a cumulative impact (Kelly, 1988). With this in mind, it is important to stress the differential impacts of sexual violence, and highlight that there is no 'one-size fits all' model for women's experiences of victimisation. While "every experience of sexual violence has consequences for women" (Kelly, 1988: 186) the impacts of sexual violence can be devastating, with both short and long-term ramifications which make it a significant public health concern (Schwartz \& Leggett, 1999; McMahon, 2010; Macy, Ermentrout \& Johns, 2011; Garcia-Moreno et al., 2012).

Some incidents of sexual violence can result in physical injuries such as bruising, scratches, genital injuries and sexually transmitted infections (Macy et al., 2011). Fortunately, most victims do not suffer serious physical injury, but this does not mean the experience is likely to be less traumatic. As well as physical impacts, sexual violence has significant psychological impacts. Victims/survivors report experiencing posttraumatic stress disorder, anxiety, difficulties sleeping, depression, difficulties with interpersonal relationships and a loss of trust (Wasserman, 2003; McMahon, 2010; Zinzow \& Thompson, 2011; Daigle, 2012; Lindquist et al., 2013). Many victims/survivors also struggle with substance-related disorders postvictimisation which can have long-term health impacts (Macy et al., 2011).

Student victims/survivors of sexual violence report similar impacts to victims generally; however the impact of academic performance may be specific to

University's student-run 'Overheard at Vic' Facebook page (Kavanagh-Hall, 2012), the leaking of nude pictures on 'babe of the day' Facebook pages by Otago University students (Elder, 2013) and the Dunedin-focused 'Rack Appreciation Society' which shared intimate sexual pictures, many taken without consent, was set up by Otago students (Weekes, 2014). 
students. Research suggests that sexual victimisation has an impact on university women's academic performance (Jordan, Combs \& Smith, 2014; Littleton, 2014), which can have consequences for subject choices, enrolment status or changes in residential status (Ottens, 2001; Lindquist et al., 2013). Academic performance as an outcome of sexual victimisation has been traditionally neglected in academic literature on university students, and further attention to this issue is warranted.

\section{Disclosure of Sexual Violence}

Sexual violence is chronically underreported, with most incidents never coming to the attention of authorities (Miller, Iovanni \& Kelley, 2011). Disclosing sexual violence is difficult and can have negative outcomes for victims/survivors. Many victims/survivors report confusion about the incident, fear of negative repercussions, uncertainty about who to tell, fear of not being believed, embarrassment or shame, and a belief that the incident was not serious enough to report (Daigle, 2012). Difficulties in disclosure among students can be compounded, as for many students, their sexual experiences at university are likely to be their first, and so the boundaries surrounding ethical sexual behaviour may not be clearly defined or understood (Fisher et al., 2009). Further, vague or conflicting university policies and definitions of sexual violence can contribute to uncertainty and make it difficult to label an incident as rape (Neidig, 2009). They may also be concerned that campus administration may 'side' with the perpetrator and minimise the incident (Kappler, 2011), leaving young women feeling confused or at fault, playing into wider stereotypes and rape myths within the university culture (Wasserman, 2003). All of these barriers contribute to a lack of reporting of sexual violence among university students.

A lack of reporting is problematic because it inhibits the possibility of victims/survivors being linked to available support services (Walsh et al., 2010; Zinzow \& Thompson, 2011). Further, a lack of reporting means that most understandings about sexual violence are based on official statistics, which may suggest that there is not an issue, and can give false impressions to stakeholders in the university about the prevalence of sexual violence (Ottens, 2001; Argiero 
et al., 2010). Such beliefs may mean that disclosures are not taken seriously, which inhibits reporting, and perpetuates a cycle of silence.

Despite official reporting rates remaining remarkably low, most victims/survivors tell someone following an experience of sexual violence (Campbell \& Townsend, 2011). Research suggests the same for student victims (Krebs Lindquist, Warner, Fisher \& Martin., 2007; Fisher et al., 2009). University students are most likely to tell their peers or friends following sexual victimisation, with US research finding that over 40 percent of a national student sample had received a rape disclosure (Paul et al., 2013). Such findings indicate the importance of delivering wider education initiatives about how to respond to a rape disclosure, to encourage those hearing disclosures to suggest the right options for victims.

\section{Responding to Sexual Violence}

In light of the confluence of factors that put university students at elevated risk, there is interest among academics about how best to meet the needs of victims and to hold perpetrators to account (Lichty, Campbell \& Schuiteman, 2008). Significant policy concerns exist in the United States regarding universities' responses to sexual violence on their campuses (Karjane, Fisher \& Cullen, 2005); however there is virtually no data about this issue in New Zealand. Despite the devastating impacts of sexual violence on victims/survivors, universities have been criticised for not responding to victims appropriately. Part of this lack of response has been argued to be in the interests of protecting institutional reputations, so as not to appear as a 'dangerous' university and deter enrolments (Fisher et al., 2009; Cantalupo, 2014).

Not only are institutions not responding to sexual violence; when they are, significant policy concerns exist with vague institutional definitions and reporting processes for students on campus in the US (Neidig, 2009). This ambiguity actively discourages the reporting of sexual violence among university women, as it can enhance difficulties in identifying an incident as sexually violent (Zinzow \& Thompson, 2011). A recent investigation into sexual harassment within New Zealand universities echoed the ambiguity in institutional reporting requirements, with the research recommending that 
tertiary institutions need to keep accurate records of harassment and assault complaints and that all complainants should be ensured confidentiality (Dunham, 2011).

Mikhailovich \& Colbran (1999: 77) argue that "by encouraging reporting and putting in place procedures to monitor reports of alleged sexual assault made to a variety of agencies, universities can be better placed to respond to those experiencing sexual assault." In this vein, it is imperative that more work is done in this area to ensure an effective and comprehensive response plan is in place. It is not possible to make conclusions about the ways in which New Zealand universities respond to sexual violence, so research attention to address this literature gap is necessary.

\section{Preventing Sexual Violence}

Such high prevalence rates among university students have encouraged myriad initiatives to be developed and implemented to address them. Rape prevention programmes have become common in high-risk environments such as universities, with most of these programmes focusing on attitudinal change on the part of victims, perpetrators and bystanders (Sochting et al., 2004; Daigle, 2012). Further, there is an acknowledgement within the literature of the importance of raising awareness about sexual violence risk, consent, and promoting ethical sexual behaviour among students (Rothman \& Silverman, 2007; Vladutiu, Martin \& Macy, 2011; McMahon \& Banyard, 2012; Moynihan et al., 2014). Challenging such attitudes is an important part of prevention, and universities are likely well-placed to develop a coordinated plan to deliver such programmes (Mikhailovich \& Colbran, 1999). Their delivery is well-documented in the US, however there remains a dearth of information about programme evaluations and outcomes ${ }^{10}$ (Gidycz, Orchowski \& Berkowitz, 2011).

As well as limited data about programme evaluations and outcomes, there remains a limited literature set regarding the delivery of self-defence training to women as rape prevention education (Gidycz \& Dardis, 2014). Recent data suggests that self-defence courses can be beneficial for victims of sexual violence in rape avoidance, and highlights the importance of women's resistance to

10 For a review of program outcomes from prevention programmes in the United States, see Vladutiu et al. (2011). 
sexual violence when the situation arises. However, there is scepticism about the delivery of self-defence training, as its provision may be seen to encourage victim-blaming attitudes (Cermele, 2004; Gidycz \& Dardis, 2014). That said, self-defence training has been shown to actually empower women when delivered from a feminist standpoint (Cermele, 2004), so its utility requires further research and development as part of comprehensive rape prevention strategies.

There remains a dearth of information regarding the initiatives currently implemented at New Zealand universities. Recent data suggests that students do not feel that their campus, including halls of residence, does enough to prevent sexual harassment (Dunham, 2013). Further, a recent Honours dissertation from the University of Auckland suggests that current prevention initiatives at the university are occurring, but are sporadic, limited in focus, have a limited reach and are limited in content, predominantly focussing on providing potential victims with information about sexual violence (Smith, 2013). In light of this, further research is needed to identify current preventive efforts and to analyse what preventive efforts would be best for different environments.

\section{Summary}

Sexual violence does not occur as an independent phenomenon; it occurs as part of a social context that condones gender-based violence. This literature review has highlighted the pervasiveness of sexual violence among university students, especially those living in university halls, and situated them within the wider social context. This review has documented the factors that elevate women's risk of sexual violence, identifying that age, elevated alcohol use, rape-supportive beliefs and revictimisation are all present within university populations. Further, this review has also highlighted the numerous negative impacts that sexual violence can have on victims/survivors, and the difficulties involved in disclosing sexual violence, which signal the importance of developing robust response protocols to encourage reporting, as well as ongoing prevention efforts to enhance women's safety at universities. Lastly, this chapter has identified the numerous literature gaps regarding sexual violence at New Zealand universities, which this research hopes to address throughout the course of this thesis. 


\section{Methodological Framework}

"By documenting women's lives, experiences, and concerns, illuminating gender-based stereotypes and biases, and unearthing women's subjugated knowledge, feminist research challenges the basic structures and ideologies that oppress women" (Brooks \& Hesse-Biber, 2007: 5)

This research sought in-depth data about women's experiences of sexual violence in halls of residence. To achieve this, I considered a qualitative research design or feminist framework was most appropriate. This chapter outlines the theoretical and epistemological underpinnings for this project, and then provides an in-depth discussion of the way the research was conducted. Further, this chapter outlines the limitations of this research which have impacted on the generalisability of the data to other groups and populations. Lastly, this chapter covers the very real and personal transformations and challenges that I have faced during the course of thesis completion. The completion of this research project has been a very personal journey and has been guided in many ways by my own worldviews, experiences and beliefs about how best to understand the issue at hand. For these reasons, this chapter is written in the first person. 


\section{Theoretical Approach}

I am attracted to the ideas of feminist research, primarily because they embrace the ideas that I have about the world. This research is therefore influenced by feminist perspectives. The feminist critique stems from the idea that women have historically been neglected by research attention, so political change and activism is needed to bring women's subjugated experiences and knowledges into light (Hesse-Biber \& Leavy, 2011). The gender disadvantage is clear for women as they are consistently underrepresented in powerful positions in society (Letherby, 2003; Millett, 2000). This power imbalance means that feminist perspectives encourage research to be for women, not on women, and with women as the key drivers and agents of change (Letherby, 2003; Allen, 2011).

When I first considered this project I was overwhelmed with the debates surrounding what constitutes 'feminist' research and was caught up in the quantitative/qualitative divide (Harnois, 2013). These debates made me question whether the research I wanted to pursue was feminist at all. Allen (2011) contends that there is not a single feminist methodology, however, Maynard (1994) succinctly helped clarify the task at hand for me as a feminist researcher, stating that "feminism must begin with experience... it is only from such a vantage point that it is possible to see the extent to which women's worlds are organised in ways which differ from those of men" (p. 14).

Maynard's ideas about where to start research led me to use feminist standpoint epistemologies for this research. Feminist standpoint epistemologies have a rooting in Marxist analyses and challenge researchers to understand and see the world through the experiences of historically oppressed groups, particularly women, and to apply that knowledge to activate change (Wood, 2009). Academics have acknowledged that sexual violence among university women occurs within a larger system of patriarchy and historically patriarchal institutions (Millett, 2000; Sloan \& Fisher, 2011; Renn, 2014). Such patriarchal values indicate the appropriateness of a feminist critique to unearth the experiences of young women living in halls and will hopefully enable their voices to be heard and be a part of social change. 


\section{Methodological Approach}

This research saw the relationship between theory and research as inductive rather than deductive, in line with qualitative methods that develop theories based on data, rather than the scientific testing of hypotheses against research findings (Hesse-Biber \& Leavy, 2011). For these reasons, a grounded theory methodology was considered most appropriate. Grounded theory is a qualitative research methodology that discovers theory through systematic analysis (Strauss \& Corbin, 1997; Glaser \& Strauss, 2012). Grounded theory involves a systematic process of collecting and analysing data; developing codes to assist with identifying themes and categories; and developing a flexible theory which can be adapted as data collection continues (Glaser \& Strauss, 2012). The method encourages researchers to constantly interact with their data, whilst remaining open to emerging analyses and changes (Bryant \& Charmaz, 2007). The systematic nature of grounded theory, as well as its flexibility, makes it attractive to new researchers (Urquhart, 2013) and useful for exploratory study.

Grounded theory is not only attractive to novice researchers - it is one of the most popular qualitative methods used across a range of disciplines (Charmaz, 2006; Bryant \& Charmaz, 2007). Such popularity has encouraged ongoing use of the theory, as well as its ongoing development and reconstruction (Bryant \& Charmaz, 2007). Charmaz has been influential in changing the direction of grounded theory, and reconstructing it to have a more interpretive rather than objectivist approach. Constructivist grounded theory is more interpretive in nature and "places priority on the phenomena of the study and sees both data and analysis as created from shared experiences and relationships with participants and other sources of data" (Charmaz, 2006: 130). Further, constructivist grounded theory highlights the flexibility of the method, which contrasts to previous versions of grounded theory which insist on a mechanical approach (Charmaz, 2014).

Sexual violence in New Zealand halls of residence is an unexplored area, so it was imperative that this study adopted a flexible approach that was able to adapt to changes in data collection and the emergence of very new themes. Further, this study required a methodological approach that prioritised participant experiences, as well as relationships between myself and 
participants. It is for these reasons that I adopted a constructivist grounded theory approach for this research.

\section{Research Methods}

\section{Initial research design}

I initially proposed a mixed-methods design, incorporating a survey and followup interviews with victims/survivors. I received positive and constructive feedback from criminology staff; however this original proposal was rejected by the School Research Committee. Whilst disheartened, I adapted my proposal to incorporate solely qualitative methods. Campbell (2002) argues that qualitative methods provide opportunities for researchers to hear the stories of survivors in ways that quantitative research simply cannot. Further, qualitative research also provides opportunities for researchers to form important relationships with participants, which leads to an establishment of trust and rapport. This relationship permits access to subjective meanings and experiences that may not be possible through quantitative methods (Liamputtong, 2007). In hindsight, using qualitative methods has been the most appropriate form of inquiry for this project.

\section{Ethics}

While all research must adhere to strict ethical standards, it is even more important when researching vulnerable populations (Liamputtong, 2007). Lee (1993) notes that the sensitivity of a topic does not determine its theoretical or social significance, however much sensitive research works to address some of the questions in society that are the most pressing but remain unanswered. What constitutes a sensitive or vulnerable population is contested as the concept itself is socially constructed (Liamputtong, 2007). I believe the population that I am researching are 'sensitive' or 'vulnerable' as this research draws on one of the most personal and distressing experiences in a person's life. Therefore, it is imperative that this research is treated with the sensitivity, privacy and ethicality it deserves.

As this research involved interviewing participants, an application for ethical approval was sought from the Victoria University of Wellington Human Ethics Committee. The application itself was comprehensive, covering a number of 
crucial areas that required consideration. Further, I also had to consider the impact a potential power imbalance between myself as a university tutor could have if a previous or current student wished to participate. I decided to exclude previous or current students from this research to avoid this. Ethical approval was granted on 20 September 2013, and subsequent amendments to the initial application were approved on 11 November 2013 and 27 March 2014 (Appendix A). The amendments requested were to conduct a paired interview with two participants from organisations under the same umbrella and to slightly change a noticed error on the recruitment flyer. The paired interview did not go ahead due to work constraints.

\section{Participants, Data Collection and Analysis}

\section{Participants}

The data for this thesis was collected from two sets of participants, key informants $(n=6)$ and female victims/survivors $(n=4)$ of sexual violence in university halls of residence. The six key informants for this research were from a wide array of professional backgrounds, with four holding positions at Victoria University of Wellington, and two working in the community. I conducted interviews with key informants to gain background knowledge prior to interviews with primary participants and to explore their knowledge of sexual violence in halls compared to the lived experiences of primary participants. To ensure the confidentiality of key informants, all informants were assigned a number in accordance with the order of which the interviews were conducted, i.e. Key Informant 1. Also, an overview of the work that they are involved in has not been provided, as by doing so it may make the informants identifiable due to the small number of staff in this area.

Primary participants were women over the age of 18 , who had lived in a hall for six months or more in the preceding five years, and had experienced sexual violence during their time in a hall. All participants were students at Victoria University of Wellington. To ensure the confidentiality of primary participants, a pseudonym has been assigned. A pseudonym was used for these participants in order to give them a sense of agency within the research and not to refer to them as 'just a number'. Some identifying details, such as the hall that women 
lived in, or different terminology used for particular characteristics in their halls, have been withheld from this research to ensure confidentiality. I will now introduce each of these women. These are written in the order by which the interviews were conducted.

\section{Indi}

Indi is a 21 year old female in her fourth year of study. She is not currently working and lived in two halls during her first two years of study. She now lives in a Wellington suburb. Indi identifies as New Zealand European.

\section{Fay}

Fay is a 20 year old female who is currently studying. She has recently finished working and lived in a hall in 2012 when she was 18 years old. She is now living in a Wellington suburb. Fay identifies as New Zealand European/Pakeha.

\section{Bianca}

Bianca is a 20 year old female in her final year of study, and working a significant number of hours in paid work. She lived in a hall in Wellington during her first year of study and is now living in Wellington City. Bianca identifies as New Zealand European.

\section{Dana}

Dana is a 21 year old female who is currently studying and working part-time. She lived in a hall of residence for two years, between 2011 and 2012. She now lives in Wellington City. Dana identifies as Pakeha.

\section{Recruiting a sample}

I interviewed women who were no longer living in a hall of residence for their own safety; I did not want participants to feel there would be dangers involved in their current living situation as a result of disclosing. Further, the decision to interview women who had lived in a hall in the previous five years was influenced by the recruitment methods which were primarily on campus.

Recruitment flyers (Appendix B) were put in women's bathrooms on campus to give women the privacy to consider the advertisement. $\mathrm{A} \mathrm{PhD} \mathrm{thesis} \mathrm{by} \mathrm{Leslie}$ 
Wright (2012) used this method successfully with university students which encouraged me to adopt it for this research.

\section{Challenges}

The use of advertisements in university bathrooms was beneficial as it allowed women the privacy needed to consider the advertisements; however the advertisements were regularly taken down in particular parts of the university. Continued monitoring and reposting of posters was time-consuming, and at times frustrating. Nevertheless, six women responded to the recruitment poster, and four were eligible to take part in the research and share their stories and experiences.

An interesting finding throughout this research was the differences between students who sent me a text message in response to the recruitment flyers, in comparison to students who emailed me. I found that students who indicated interest by text message were less likely to take part in the research and more likely to cancel at the last minute. Three out of four women that took part in this research had indicated interest via email, so were able to consider an information sheet and consent form prior to participating. This may have been influential in their decision to take part and could be considered by other researchers in this area.

The eligibility criterion meant that two women were unable to take part in this research. The first person who contacted me about taking part in the research was not eligible as the rape she experienced was nearly thirty years ago, thus it sat outside the scope of eligibility criterion. I feel it is important to validate her experience here, and the possibility of historic sexual victimisation in the context of hall environments should be considered for future research.

The second person that contacted me about the research had only lived in the hall for three months and moved out following an attempted rape, which she disclosed during an informal discussion over coffee. I would have loved to include her experience here, and had not initially considered that someone may have been dislocated from the hall so soon after moving in. This significant oversight should inform future thinking about how research parameters are established with university students. 


\section{Data Collection}

Qualitative, face-to-face, semi-structured interviews were used in this study. The interviewing of key informants and primary participants was expected to be a two-stage process. In practice, the difficulties involved with scheduling interviews with people leading very busy lives meant that there was some overlap. Interviews with key informants were conducted between November 2013 and May 2014. Prospective key informants were invited via email to take part in the research and were identified through existing social networks. Eight key informants were contacted, and seven agreed to take part, however only six went ahead due to work constraints. One key informant did not respond to email invitations. All key informants were provided with a copy of the key informant information sheet and consent form (Appendices C and D) in the initial email. Interviews were scheduled at times convenient for participants and took place at their places of work. All key informants were introduced to the research and signed a consent form. Permission was also sought to audio record the interviews. Questions were asked using a semi-structured interview schedule (Appendix E) however questions remained flexible in line with the informant's expertise or knowledge in the area. Interviews averaged 45 minutes in length.

For primary participants, advertisements were placed in bathroom stalls around campus and contained my personal details for participants to contact me. Interviews with primary participants took place between March and April 2014, averaging a length of 67 minutes. There was a significant delay in recruitment as most students are not on campus during the summer break. This meant I had to wait until students returned for the first trimester. The time and location of the interview was largely left up to participants to decide, as it was important for me to ensure participants felt comfortable enough to participate in an interview on such a sensitive topic, and to attempt to reduce the power imbalance between myself and participants. Interviews took place during the university day, with two interviews in a private room on campus, one in a private room in the library and one in a university café.

Each interview commenced with an overview of the research and reiterated the importance of ensuring confidentiality. Participants read over the information 
sheet prior to the interview commencing (Appendix F). I sought permission from participants to audio record the interview and gave participants the opportunity to ask any questions about the research before signing the consent form (Appendix G). Using a semi-structured interview guide (Appendix $\mathrm{H}$ ) helped to provide some key points of discussion during the interview and did not dictate how the interview was run as discussion was primarily led by participants.

During interviews, I took the approach of a researcher, but retained my status as a female student and previous hall resident as well, which I felt gave the women a sense of ease in disclosing to me. Due to the sensitive nature of what was being discussed, it was imperative that my approach was empathetic and sensitive, but at the same time maintained a sense of awareness in order to understand how they interpreted their experiences. I remained conscious that, both as a student and a previous hall resident, I did not give the impression that I was familiar with their unique experience, but at the same time I ensured the women knew that their experience was valid. At the end of the interview, all participants were asked if they had any further comments they would like to make, which helped with interview debriefing. All participants were provided with a sheet of available support services in an effort to minimise the harm of participating in the research (Appendix I). Finally, all participants were given a \$25 New World voucher as a koha for their time.

\section{Data Analysis}

All interviews were transcribed verbatim from audio recordings. The transcription of ten interviews was time consuming, but useful as it helped me become more familiar with my data and consider emerging themes. All participants were provided with a copy of their interview transcript and were invited to make changes if they wished.

As previously discussed, this research uses constructivist grounded theory which consists of many of the same conceptual underpinnings as grounded theory, but within a constructivist approach (Charmaz, 2006). Grounded theory encourages researchers to write memos which provide an immediate illustration for an idea and are useful for comparison of substantive areas later in the 
analysis of data (Glaser \& Strauss, 2012). In line with these concepts, memos were written between coding and writing. Further, constant comparisons were made, and questions asked, about the data, codes, memos and categorisations of the data. The use of memos was helpful during and after interviewing as it allowed me to record important expressions and tones which are not always as evident in transcribed form.

Initially, I began coding line-by-line in paper form, using highlighters for emerging themes. This task became overwhelming, so I began coding using NVivo qualitative data analysis software. NVivo proved invaluable. I coded my interviews line-by-line and then used the codes to identify and assess emerging themes. The findings were compared and discussed with existing literature on female students' experiences of sexual violence at university, particularly in halls. This analysis revealed the unique hall climates that women were living in and their experiences as a result of such climates, which has not previously been discussed in-depth in New Zealand literature.

\section{Presentation of data}

The final write up of this thesis occurred over the spring months of 2014. This thesis has taken a total of eighteen months from initial enrolment until completion due to health and recruitment related issues which necessitated six months part-time status and a three month extension from September until December 2014. All participants provided contact details for the return of a copy of the finished thesis. A copy will also be provided to the New Zealand Family Violence Clearinghouse in fulfilment of scholarship requirements.

\section{Limitations}

The limitations of this research include the small sample size $(n=4)$ which has impacted on the generalisability of the data. However, the qualitative design used for this research did not set out to identify data that would be generalisable; rather it sought to identify women's subjective experiences of sexual violence and these subjective experiences are a valid and important part of research.

A further limitation of this research is the focus on cisgender females and the exclusion of men. Trans women were not included in this research as no trans 
women came forward to participate. The exclusion of men from this research was a decision made based on two factors: the limited scope of a Masters and the lack of literature about sexual violence in halls generally. The exploration of men's experiences would be a valuable area of inquiry in the future.

The focus of this study on hall populations - a population typically represented by young people in emerging adulthood ${ }^{11}$ - has meant that the views of older women have been excluded from this research. Research with older women, who are living in halls, perhaps as postgraduates or international students, could be an interesting area of future research.

Lastly, all of the women in this research identified as New Zealand European or Pakeha. The small sample size has undoubtedly impacted on the inclusion of intersectionalities such as race, ethnicity and sexual orientation, which were unable to be explored. Whilst exploring the influence of such factors on women's experiences would have been an interesting area, the small sample size $(n=4)$ and the cultural issues that can impede sexual violence disclosure among ethnicities ${ }^{12}$ has impacted on the representation of women included in this sample.

\section{Personal challenges and transformations}

The completion of this thesis has been a very personal and transformative journey, which has posed a number of challenges throughout that I believe should be mentioned here. The first challenge was not having my original proposal accepted. It was disheartening and made me question my ability to study at Masters-level. However, it is important to take such things in stride, which is one of the things that completing this Masters has taught me - there is no right way to do things.

One of the biggest challenges during this thesis was acknowledging the impact that researching sexual violence had on me as a researcher. I studied sexual violence during my undergraduate and Honours years and I had not felt affected by it previously. However, the study of sexual violence at such a real level, with

${ }^{11}$ Emerging adulthood refers to the developmental period between the ages of 18 and 25, and is described as "a period of identity exploration, instability, self-focus, transition and possibilities" (Clodfelter, Turner, Hartman \& Kuhns, 2010: 456).

12 See Fontes \& McCloskey (2011) for a discussion of cultural issues in examining violence against women. 
women of a similar age to myself, triggered a set of emotional and physical impacts, such as violent dreams and a heightened risk-awareness state in normal social situations. Rebecca Campbell's (2002) book about the impact of researching rape had not previously been a book on my shelf, but it now sits right at the top and has been of huge assistance in getting through this thesis.

I had also not anticipated receiving a phone call from a woman in crisis who was triggered by my recruitment posters. The feeling of making someone so upset for my own research gains was difficult to grapple with, however the support from my supervisor during this, and a belated understanding that feeling triggered can actually be a good thing for some survivors, helped to ease the upset I felt I had caused. 


\title{
Expectations, Risks and Realities: A Background to the Findings
}

\author{
"Living in a hall can be complex. A lot of people will love it, some \\ people will hate it” (Key Informant 6)
}

\begin{abstract}
Although only four interviews were conducted with women who experienced sexual violence in their halls, the amount of data within these interviews canvassed a number of key issues and themes. Further, interviews with key informants also provided a rich data set. This chapter explores three key themes that I identified from interviews with both key informants and primary participants. These themes related to the expectations of hall environments and life in a hall, touched on by both women and key informants; the risks students face living in halls of residence identified by key informants; and the translation of these risks into reality for the women interviewed. The lived experiences of the women interviewed for this research highlight a number of incidents that they experienced directly, or that they reflected on, in the environment around them. The coverage of these three themes serves as a platform to view subsequent chapters.
\end{abstract}




\section{Life in a University Hall}

Historically, universities did not consider the importance of social integration and relationship building as conducive or relative to academic success for first year students. In fact, the success of first year students used to be viewed solely as a result of their unique academic qualities and social privilege (Greenfield, Keup \& Gardner, 2013). Increasing attention has been paid to understanding the unique first-year experience internationally since the 1960 , emphasising the importance of universities adapting to the needs of first-year students (Zepke et al., 2005; Johnston, 2010). In New Zealand, nearly half of all full-time students do not complete the degree they initially enrolled in, and a substantial number of these students withdraw from study in their first year (Scott, 2009). Such figures present a unique and significant economic problem for New Zealand tertiary institutes (Walker, 2010). Despite this, there remains a lack of research in the area of student retention and the provision of support to first year students in New Zealand (Prebble et al., 2004; van der Meer, Scott \& Neha, 2010).

The transition to university living is a difficult one for many students, often signalling the end of childhood and the beginning of life as an adult (Game \& Metcalfe, 2003; Gengler-Dunn, 2007; Walker, 2010). However, Prebble et al. (2004) note that when environments and processes are welcoming for university students, student outcomes are likely to be enhanced. That said, available and accessible student housing is an important responsibility for universities as it adds to the community feel of the campus and the overall quality of life for students (den Heijer, 2011). Zeller (2005: 410) argues that "where first-year students live matters" and that the support systems and experiences within residential hall environments are highly influential for firstyear students' educational outcomes. Paine (2008) agrees, contending that students living in halls are more likely to become more integrated into the social and academic systems of the university, which can positively affect students in other ways.

The decision to study at a particular university can be influenced by the student lifestyle and residential environments it provides. A recent Master's thesis suggests that this was an influence for Otago University students, who 
commented that the close communities offered by flats and halls of residence at Otago were attractive (Walker, 2010). The transition to university living undoubtedly arouses feelings of uncertainty (Walker, 2010), a sentiment that has been echoed in my own research. Although the transition to hall life was not a central focus of this thesis, three participants commented on their expectations of the hall environment prior to moving in. For these women, expectations of integration, particularly in social systems, were strong:

I thought it was quite good to go to a hall for like my first year... it's a good way to get to know people, get a feel for the city (Indi)

I had never really lived with males before. I went to a boarding school which was 150 girls I lived with for 5 years. So I thought living in a hall with guys would get me ready for flatting (Bianca)

Fay also commented on her feelings of uncertainty about what the hall environment would provide for her, identifying that "with halls you never really know what kind of lot of people you're gonna get". This uncertainty is common, as the move to university is such a significant one for most young people (Game \& Metcalfe, 2003; Gengler-Dunn, 2007). The way that participants described their expectations of the hall does not differ from the literature around the firstyear student experience. However, as this chapter will later highlight, particular incidents and events that occurred during their time living in a university hall have contributed to some participants having negative memories and reflections on their hall experiences.

\section{The influence of American fraternity imagery?}

The American fraternity image of a sexualised university environment with copious amounts of alcohol and other drug use permeates popular media. Sanday (2007) vividly describes this environment in her overview of campus party culture in the US and its associations with fraternity gang rape. Given the dominance of such images in the media, it is likely that these would influence New Zealand university students' expectations of hall life. One key informant commented on the power of this imagery:

And I'd also say that a lot of those kind of cheesy American college representations of life while living at university sort of come up with a bit of a folklore image about what living in a hall is all about. And there are some elements of truth in it. But there's a much vaster list of things that's not necessarily represented (Key Informant 6) 
Another key informant also reflected on the expectations students might have when they come to university, drawing similarities between Orientation Week $(\mathrm{O}-\mathrm{Week})^{13}$ in New Zealand and the fraternity scene that dominates American popular culture:

There's also this expectation that they come to university, and especially during $\mathrm{O}$-Week. Or they go to all these universities in $\mathrm{O}$-Week. It's like sex is free and on tap. Alcohol is free and on tap. Drugs for free and on tap kind of thing. And it's like throw all that in together. It's like the Sevens or whatever else (Key Informant 1)

There is a point of difference here between key informants' identification of the influence of party culture and the expectations put forward by women interviewed. Of the women who commented on their hall expectations, none of them discussed their expectations being in line, or influenced by, the party lifestyle. However, this does not mean that parties and alcohol use did not become part of their lived realities in halls, as this chapter will later discuss.

\section{Risk in Halls of Residences}

As previously discussed in Chapter Two, university women are at an elevated risk of experiencing sexual violence (Fisher et al., 2000; Nasta et al., 2005; Gross et al., 2006; Walsh et al., 2010). Some research even suggests that young women living in university halls may be at an increased risk (Katz et al., 2010). All key informants were asked about what they felt may put university students, particularly hall residents, at risk of sexual violence. These risk factors will now be explored in detail.

\section{Mixed-Gender Living and Public Space}

Living in a university hall is common for many first year students, with most residents spending the majority of their free time outside of campus at their hall. Grigsby (2009: 57) stipulates that "for most the residence hall is the first place they inhabit and share with peers at [university] and is very important in establishing a sense of what the shared college student culture is for them." While the hall provides an environment to achieve that, living alongside other students in such a 24/7 environment means "there's a real difference in terms of what you even see of people” (Key Informant 5). One key informant

\footnotetext{
${ }^{13}$ Orientation Week is the week prior to the first week of university for students in New Zealand.
} 
commented on the differences associated with living with others in a 24/7 environment compared to other non-residential settings, noting that "as soon as you have a residential setting, of whatever sort, because people are living there... the boundaries are really different in how they behave" (Key Informant 5). This idea of 'seeing more of the person' has not been previously discussed as a potential risk factor in literature regarding sexual violence in halls and could help inform future research questions or parameters.

All key informants commented on characteristics of hall environments that are risk factors for students in halls. This statement is echoed by Fisher and Wilkes (2003) who acknowledge the increased risk in halls posed by proximity-tooffender situations. Two key informants commented on this specifically:

...living in an environment where a lot of the halls have got mixed floors, they've got mixed shared bathrooms. You know there's a lot of public space so those issues might potentially make it harder to create a safe space for yourself, or claim a safe space (Key Informant 3)

...the moment you have halls that have got mixed genders, yknow with a highly kind of sexualised population at a massive freedom risk-taking time of their life, there's going to be lots of sexual yknow conduct taking place (Key Informant 4)

As well as the dynamics involved in mixed-gender housing, two key informants also commented on the lack of safeguards for students living in halls in comparison to living in the parental home:

There aren't many formal structures in place to protect them. I mean I guess people have got a lock on their bedroom door... I mean I think for a lot of young people who come to university, who are living away from home, they will be living quite differently to the way they lived at home, and so, potentially, things which kept them safe almost unconsciously won't be there (Key Informant 3)

The lack of safeguards in university halls, in comparison to previous parental living environments, has been acknowledged by many researchers as a risk factor for sexual violence for students living in halls (Payne, 2008; Neidig, 2009; Katz et al., 2010). The comments provided by key informants here correlate with the existing literature in this field, yet provide a New Zealandspecific account which has not existed before. This New Zealand account is important given the differences between the New Zealand residential hall 
environments, and the fraternity and sorority culture that dominates US literature.

\section{Substance Use}

Increased autonomy is characteristic of the transition to university life, and Paine (2008) suggests that this is a risk factor in itself because it gives students the opportunity to participate in high-risk behaviours such as increased consumption of alcohol. The links between alcohol and sexual violence are very well documented, and there is an abundance of literature exploring the links between alcohol use and sexual violence within student populations (AdamsCurtis \& Forbes, 2004; Cashell-Smith et al., 2007; Connor et al., 2010). All six key informants commented on alcohol as a contributing risk factor for student populations:

Alcohol is always a factor. I guess, for both perpetrator and victim in terms of the victim's ability to see an unsafe situation coming... I don't like to see alcohol as the issue, but it's a contributing factor (Key Informant 3)

I would consider [alcohol] an exacerbating risk factor because I know men are victims of violence but we're predominantly dealing with female victims in this context. That alcohol can be used as a means of victimising a woman. So if we've got a high level of alcohol use in a population of young people, then that's creating a situation where there are opportunities to victimise people (Key informant 6)

Although the link between sexual violence and alcohol is supported by a substantial body of evidence, more research is needed that focuses on the role alcohol may play in victimisation in halls of residences.

Some research has explored the role of other substances in drug-facilitated sexual violence among university students (Kilpatrick et al., 2007; Krebs et al., 2009). One key informant commented that the use of other drugs has not featured prominently in their work with students in a response setting. Key informant 4 notes that "anecdotal of the cases I've been aware of, alcohol is often involved, yknow. Often, often, often. Not other drugs particularly, just alcohol." This observation provides some indication of the circumstances surrounding New Zealand students' victimisation in the absence of research on the issue. It may be possible that the lack of involvement of other drugs in the facilitation of sexual violence - or the lack of knowledge of its involvement among students - is related to the actual use of a drug to incapacitate a victim. 
The use of drugs such as Rohypnol and GHB, which have been referred to as 'date rape' drugs, can make victims lose consciousness and render them unable to recall events (Fitzgerald \& Riley, 2000; Fisher et al., 2009). Whilst such comments are speculative at best, it does signal an area that requires more research attention, particularly in the New Zealand context.

This discussion has shown that university hall environments contribute to a degree of elevated risk for residents. From these observations, it is deducible that the combination of public space and mixed-gender housing, in conjunction with increased alcohol use within environments that lack previously existing safeguards, contributes to elevated risk of sexual violence.

\section{Hook-up Culture}

The term 'hook-up culture' is frequently referred to in academic literature, particularly with regard to university populations. 'Hooking up' does not have a precise meaning; however Bogle (2008) identifies that hooking up is a broadly definitive term that encapsulates a wide array of sexual experiences and intimate interactions. Hooking up, therefore, is the:

dominant way for men and women to get together and form potential relationships on campus. This does not mean that everyone on campus engages in hooking up; but students do consider it to be the primary means for initiating sexual and romantic relationships (Bogle, 2008: 37).

In Bogle's (2008) analysis of the emergence of a hooking up culture, she argues that the shift away from traditional dating and the move toward a more casual approach of socialising and getting together occurred in the 1960s. According to Bogle (2008), this shift in social interaction in the university environment is the result of a number of sociohistorical trends such as the increased availability of contraception, liberalisation of attitudes toward sexuality, the women's movement and the dramatic increase in the numbers of women attending tertiary institutions. Two key informants commented on the changes noticed in sexual interactions among university students, reflecting on the transactional nature of sexual behaviour:

The kind of commodifying of sexual interaction I've noticed change. It's always been there amongst some, but I'm surprised at how transactional sex seems to have become amongst a lot of students, including a lot of students in halls... the kind of hooking up mentality is, is pretty prevalent, 
I think... I'm kind of amazed at the casualness with which quite a lot of students talk about their relationships (Key Informant 4)

Two key informants found this notion of hooking up to be problematic, and one key informant refers to the term "friends with benefits", described as constituting:

this perception that that we're just friends but we're sleeping together but we're not in a relationship when we're sleeping together. It's the most bizarre uh, you are in a relationship end of story. You're in a sexual relationship. You may choose for it not to be just with one person but acknowledge that you are (Key Informant 1)

The existence of a hook-up culture in university halls has been identified by key informants as a contributing risk factor for sexual violence. This finding could be beneficial in the consideration and development of sexual education and sexual violence prevention information delivered to hall populations, or the wider student body, to ensure relevancy to the changing nature of sexual relationships among university populations.

\section{Sexual Pressure and Navigating Consent}

It is important to acknowledge the role that social pressures within particular cultural contexts can play in contributing to elevated prevalence rates among university populations. Schwartz and DeKeseredy (1997) provide an overview of male peer-support theories of sexual assault on university campuses, arguing that a socialization process exists on most campuses which means men expect to engage in a very high level of consensual sexual intercourse. However, for the most part, these goals are "almost impossible to achieve" (p. 34), In this way, when men are gathered together in spaces such as university halls, the need to reinforce interactions to strive for hegemonic masculinity is profound to avoid ridicule and harassment (Argiero et al., 2010). With this in mind, sexual violence occurs during the attainment of this goal for superior status within male hierarchies at university. Four key informants discussed sexual pressure within hall environments as a contributing risk factor for sexual violence, and Key Informant 6 identified the problems associated with students' perceptions of wanting to be sexually desired, commenting that such perceptions may not be met and "people are victimised as a result." Key Informant 5 acknowledged the 
dangerous consequences of environments that localise sexual pressure, stating that "rape in that context isn't then surprising" (Key Informant 5).

Another concern identified by key informants regarded the difficulties involved in navigating consent among student populations. One key informant commented on the 'illiteracy' of New Zealand's sexual culture which, coupled with most sex occurring in a non-verbal environment, highlights "where the road to misunderstanding and issues of consent being kind of fudgy are rife" (Key Informant 4). Key informant 6 felt similarly, and expressed that "there would be an at-risk situation perhaps where people, and I'm talking about guys here, don't understand boundaries in relationships." These observations help identify components that could be useful for incorporation in sexual education for students in halls.

As well as sexual pressure and a lack of respecting sexual boundaries, it is likely that stereotypical ideas about rape contribute to students' difficulties in navigating consent. The presence of rape-supportive beliefs in society is an area of focused and dedicated research attention, with much research exploring rape myth acceptance among university students. ${ }^{14}$ Two key informants reflected on the presence of rape myths within university hall populations and the problems that such beliefs have:

Perhaps they are perceiving sexual violence as that rape myth of stranger danger, or these things are gonna happen outside my hall or whatever it might be. (Key Informant 6)

[The] first year student at whatever hostel or hall doesn't look like a rapist... there is something more potentially vulnerable for people there because how we work in society seems to be kind of around this idea of identifying the people who are creepy or whatever... one it's not rape because he doesn't look like a rapist. Two it's not rape because I was so drunk (Key Informant 5)

The changing nature of sexual behaviour among student populations, coupled with an environment of sexual behaviour and a culture that perpetuates societal misunderstandings about rape, can contribute to risk in hall populations. The difficulties identified around consent open up potential avenues for education in the future with university hall residents, as "people need to know more about

${ }_{14}$ See Suarez and Gadalla (2010) for a meta-analysis on rape myths, including many university samples. 
consent. I think that is something that really needs to be thought about" (Key Informant 2).

\section{Realities of Living in the Mix}

\section{Environment and Public Space}

Key informants commented on the risk young women face in university hall environments, and these understandings are important to consider in tandem with the lived experiences of women in halls. Three of the women interviewed commented on what living in the hall was like and some of the common activities that occurred in these environments. Three participants identified similarities between their hall environments and the atmospheres within boarding schools or high schools:

I didn't really like the environment, it was kinda felt like some sort of boarding school. I expected it to be a bit more, ah, open-minded, weirdly? Does that make sense? I dunno, it seemed quite like childish (Fay)

It's actually surprisingly similar the culture, from about year $12 s$ and year 135 in a boarding school as it is in a hall. The cultures are actually very, very similar... Less studious than year 12 and 13 [laughs] (Bianca)

Fay called the hall an "environment for sex" and two of the women commented on the ease that information about residents' sexual histories became public knowledge:

Sitting in common areas and gossiping was a big thing they [residents] did. Like who's fucking who, someone failed an assignment or something like that. Petty, petty things from petty, petty people [laughs]. That was a common thing (Bianca)

Well like, if someone had sex with someone, everyone would know in a matter of time and then you'd like identify who'd had sex with who and the emergence of that kind of thing. And when you're all living together, I dunno it's just quite interesting (Fay)

These observations echo the commentary provided by Key Informant 6 about victimisation when privacy has been lost in a hall:

I'm talking about victimisation where the loss of privacy for example in a hall where perhaps somebody might feel victimised because their sexual history, or things they've experienced in a sexual context, are common knowledge. That must be a very difficult thing for young university people, for both guys and girls. I can't imagine what that would be like now... there is no privacy (Key Informant 6) 
The loss of privacy regarding intimate histories is a difficult issue for students to manage, and the impacts of this loss could be exacerbated in a public environment such as a hall of residence. Education around the importance of respecting other residents' privacy could be beneficial for university hall management to consider.

\section{Substance Use}

Alcohol consumption among students is generally viewed as a rite of passage (Sloan \& Fisher, 2011). In New Zealand, alcohol is viewed by some as intrinsic to the university experience (Towl, 2004). Further, New Zealand data suggests that alcohol use within halls of residence is amplified, and students in halls are more likely than other students to report frequent binge drinking (Kypri et al., 2007; Kypri et al., 2009). The acculturation of heavy drinking within university halls (Towl, 2004) is concerning considering the documented links between alcohol and sexual violence, especially among students (Abbey et al., 2003; Adams-Curtis \& Forbes, 2004; Connor et al., 2010). Three participants commented on the role alcohol played in potentially non-consensual sex in their halls. Bianca draws similarities to the Roast Busters, where a group of young men were allegedly involved in the group rape of intoxicated, underage girls:

Half the time, the girls that did get victimised were under the influence of alcohol, like to the point of blacking out. It was kind of like the spit roasters up north almost, ergh. Like getting the girl so drunk that she will put out, it was actually disgusting to hear about even (Bianca)

Indi recalled an incident where a resident had sex with a girl who was too drunk to consent and Fay also remembers situations in her hall "where a girl would be really drunk and then be in a boy's room and then people would go and get the [Residential Assistant]15 to get the boy out." Fay's account highlights the important role that bystanders can play in preventing sexual violence from occurring when dangerous situations are identified.

Overall, participants identified a number of ways in which the risk factors discussed by key informants manifested into lived experiences within halls. However, one participant discussed helpful advice given at the start of the year

15 Residential Assistants are senior students who are employed to provide support to other students and assist with the day to day running of the hall. 
regarding keeping herself safe which highlights the active role her hall played in educating students about potential risks:

Yeah we got taught about like don't let drunk girls go home by themselves, or drunk boys for that matter, but yknow. Um if you can see something happen, interfere. I don't know, yknow basic safety things... I think that was really good of the university to implement that because I think when a lot of people are 17 or whatever and they haven't been allowed to do anything at home and suddenly they have all this ridiculous amount of freedom, and then it's good for you to give them a bit of back down to earth kinda thing (Fay)

The provision of such advice at the beginning of the academic year was really helpful for Fay, and it is promising to see that delivered in a hall of residence. As this thesis will discuss in later chapters, the importance of equipping students with the information and education about what to expect in the hall environment and how to be a good bystander is paramount.

\section{Participant's Experiences and Knowledge of Sexual Violence in Halls}

As this research focuses on women's experiences of sexual violence in university halls, all of the women were asked to comment on their experiences or observations of such violence. All of the women discussed their own experiences in detail, as well as reflecting on other incidents that occurred in their hall that they were aware of or witnessed, which provides an interesting lens by which to analyse the overall hall climate. The women's experiences of sexual violence had profound impacts, so it is important to provide an overview of the types of incidents that occurred in their halls, in order to contextualise the discussions in later chapters.

\section{Sexual Violence Experiences and Reflections}

\section{Indi}

Indi lived in two university halls and described ongoing comments and jokes by others that should not have been made. Indi recalled that such comments and jokes were really common with one particular resident, and things got out of control with his behaviour, especially in second year. Many of his comments occurred in public, and other residents excused his behaviour by justifying it as his sense of humour. Indi remembers one incident particularly vividly which occurred when a big group of residents were in a double room: 
And I think I said something like 'oh wouldn't it be funny if whatever, I don't know, this happened' wouldn't that be funny or whatever. And he was, like completely irrelevant, was like 'yeah, you know what would be really funny? If everyone just left the room and like, you got like dressed up in leather and a whip or whatever and we just had sex'. And I was like 'okay'. And I just didn't like know how to respond to that.

His problematic behaviour continued in second year at her next university hall as the student became involved with her group of friends. Indi felt that her friends who were in a relationship had an "aura of protection" where he could make the odd joke to them, but she felt that being single meant he could "take it that one step further" with her.

\section{Fay}

Fay lived in a university hall in her first year. Fay experienced numerous incidents during her time living in a hall. The first incident she recalled was sexual comments made by a Residential Assistant:

And there was one [Residential Assistant] in particular who would make like extremely sexual comments to me and he knew my cousin... he's also telling my cousin, who I think's really cool, a lot of this kinda stuff, it made me feel really uncomfortable.

As well as the sexual comments from her Residential Assistant, Fay recalls an incident in that latter half of her first year which had an ongoing personal impact:

I like did something sexual with someone in the bathroom of a club, or whatever. Which, yknow, I'm sure many people have done. Unfortunately, this wasn't someone I knew very well. And he went to my hostel. And he told people. And by the next day, everyone knew. Like everyone knew... there was like an incident where I once walked up Kelburn Parade and this girl was like, in a big group, and she was like 'there's that slut Fay'... So that was bad enough that week afterwards, where people were like genuinely saying 'slut' like loud enough, like audibly in my presence.

As this became public knowledge, some boys at the hall encouraged a tutor to use the scenario of a sexual act in a bathroom becoming public knowledge as the subject of a problem tutorial question for a group study programme at the hall:

And then they had this problem question... the main part of it was about me [doing a sexual act] in a bathroom. It wasn't specifically me, but yeah ... absolutely everyone in the room knew it was about me. So like 
everyone just looked at me. And I couldn't leave coz I was like in the middle of the room.

The following year, the stigma from her first year hall continued at an organised university retreat:

... as I was in the bathroom I could hear outside, and there was this one guy [name redacted], he was talking about me to people who hadn't gone to my hostel, so he used my full name and then he said yeah, she's a huge slut.

\section{Bianca}

Bianca lived in a hall in her first year, and her own experience of rape during her high school years heightened her awareness of potentially risky situations around her. She remembered a situation on her first night in the hall, and admitted that she still felt confused about whether a guy had actually come onto her:

There was another guy, on the first night I'd basically spilt my whole guts because I was homesick and wanted someone to talk to. And, I mean he it's difficult to say whether he actually did come onto me. He never, I know he's flirtatious by nature [laughs] and he was well aware I had a boyfriend and everything like that, but thankfully he moved on pretty quickly.

Bianca also recalled a student with a reputation for trying to get sexually involved with residents in his room trying to hit on her in a public space:

Well the creepy guy that used to drag people into his room to study, he tried to talk to me once... I was sitting next to him... all I could think was 'get away from me, get away from me, I don't want to talk to you, I know what you're doing, get away from me, even four feet away you feel slimy'. And he was just trying to talk study with me and I just I wouldn't have it. I think I was one of the few women who refused to go and study with him because I knew what was happening. I could feel the creepiness I suppose coming off him. It kind of radiates.

\section{Dana}

Dana recalled a number of things happening during her time at the hall. She described being filmed in the shower at her hall:

The biggest thing was that in the summer of 2011, at one point I was taking a shower in a house. And for some reason I looked up, and I realised that someone was filming me, on their phone... I went and told my friend who was on duty about it and he called the Police. Police filed a complaint and everything. 
Dana also recalled a resident who became obsessed with her:

... he became interested in me and was very convinced that however nice he was to me was proportional to the amount of sex that I would give him later, pretty much. Like he would buy me chocolate and then complain that I wasn't going out with him. Or he would like hug me and then just kinda make a comment that obviously I'm so caring, we should go out at some point. And he did - a lot of it was just comments... But at one point he picked me up in a busy room and my top came up. And so I frantically pulled it down and he just kind of laughed it off. And he was doing this all in like really public places in the hall.

She remembers some of the comments being quite violent, particularly once she became involved in a relationship with her partner in second year. Dana noted that "he wanted to have sex with me with a dentist drill and it would be hilarious... I remember at that time being like woah, this is officially going too far."

There are a number of similarities in the women's stories, particularly regarding ongoing and unwanted sexual attention. The same can be said for their reflections on the hall climate. Indi reported that the relations between men and women in her halls were for the most part "alright... There were some things that weren't really okay, I guess. Some relationships that probably weren't so healthy." As well as problematic relationships, there were a number of problematic residents that participants identified, as well as problematic attitudes and behaviours within the hall. Two participants reflected on alleged rapes that had occurred in their residence that they were aware of:

And it didn't happen to me, but beforehand during the previous year, there was a very serious allegation of rape between two people. And I didn't believe her at the time - and I feel awful now - but I didn't quite believe it at the time from what she was saying (Dana)

Dana recalled the victim in the alleged incident discussing the way that the hall staff were covering up her allegation, which made Dana not believe her. It was not until she herself encountered hall attitudes toward sexual violence that she was able to believe the previous victim.

Fay also commented on common incidents within the hall which contributed to a sexualised environment:

There's just a lot of, quite a lot of groping or sexual comments or whatever, or like ratings systems. Groping was kind of like a more on the 
way to town or in town thing. But people from your hostel grope you in town all the time. Women get groped all the time in town. I have been groped from behind, like vagina-grabbed, in town... And then ratings systems like guys would like rate the hottest girls in the hostel or people would like draw diagrams of like who's had sex with who and stuff like that. Constant scrutiny thing about how hot you look or whatever.

Bearing all of these experiences in mind, Dana sums up how she viewed the hall environment rather succinctly, stating that there was "a lot of really misogynist, sexist stuff going round. Like yeah. Everyday when I walked into lunch there would be some sort of comment at some point. There was just way more casual sexism."

\section{The Framework for the Following Chapters}

The notion that university hall climates are overtly sexualised has been clearly depicted throughout this chapter, and the comments and observations presented provide an important lens to view subsequent chapters through. Undoubtedly, such sexualised hall climates, and students' experiences of sexual violence, had profound impacts for the women living in them. The following chapter explores the impacts that such experiences had on their lives, some of which have continued in the long-term. Following on from the impacts of sexual violence, this research then addresses the disclosure of sexual violence by participants, and identifies the factors that contributed to their decision about whether to disclose. This research then turns to the response protocols that are currently in place for victims/survivors in the event of a disclosure, such as the help available for women post-assault or experience, and examines women's experiences with responses in the event of formal disclosure, and the reasons behind women's decisions to help-seek or not. Lastly, this research explores what can be done to improve responses to, and ultimately prevent, sexual violence within university halls, incorporating the views of both victims/survivors and key informants as part of these recommendations. 


\section{Sexual Violence and its Consequences: The Impacts of Experiencing Sexual Violence}

Yeah it's funny coz like it was a huge impact. But now that I'm so far away from that situation, like it's hard to actually remember how awful I felt at the time, if that makes sense? (Indi)

The ways that women respond to experiences of sexual violence are highly individualised and complex (Kelly, 1988). Some women may experience a high level of trauma across a range of different levels, whereas others may not feel affected at all (Yuan, Koss \& Stone, 2006; Basile \& Smith, 2011). Despite these differences, there are a number of impacts that many victims/survivors experience and a number of these were experienced by participants in this research. This chapter analyses the impacts of sexual violence experienced by the female students interviewed during their time living in halls. For the purposes of this chapter, the impacts that women experienced are divided into three sections. The first explores the psychological impacts and consequences that participants reported, which affected them in both the short and long term. The second section explores the impacts that their experience had on their social worlds. Finally, the third section explores the academic impacts reported by one participant that makes a significant contribution to the literature base on sexual violence and educational outcomes. Although the sample size is small in quantitative terms, the stories that women told are a useful qualitative complement to issues identified elsewhere in larger studies. 


\section{Psychological Impacts}

Whilst experiencing sexual violence makes women vulnerable to experiencing short and long-term consequences, the immediate impacts can be particularly distressing and may include feeling fear, anxiety, confusion and social withdrawal (Kelly, 1988; Yuan et al., 2006; Calhoun, Mouilso \& Edwards, 2012). It was not until the 1970s that empirical studies of the psychological impacts of sexual violence began to appear in research literature (Petrak, 2002), however these impacts are now well-documented (Briere \& Jordan, 2004; Calhoun et al., 2012). Understanding why one person may develop a particular response to an incident that differs vastly from another victim/survivor is typically linked to a diagnosis of a particular symptom or syndrome, such as posttraumatic stress disorder, rather than differentiating between different symptoms, syndromes and responses (Briere \& Jordan, 2004). Accordingly, this research does not attempt to understand the reasons behind women's unique postvictimisation outcomes; rather, it explores these impacts and responses in detail and aims to understand the context and environment within which they occur. This chapter explores the personal, social and academic impacts that sexual violence had on the women interviewed.

\section{Affecting 'the self'}

Experiencing sexual violence can have a profound impact on a victim/survivor's self-schema, and numerous researchers have explored the impact that sexual violence can have on 'the self' (Huerta et al., 2006; Calhoun et al., 2012). All participants reported that experiencing sexual violence in their hall affected them in a very personal way. For Fay, with everything happening at the hall at the time, she completely "lost all confidence in things." Further, Fay also reported that she felt a complete loss of control when a Residential Assistant at her hall made comments about her, in many ways because of the power imbalance between them:

And then he'd make ones about me like being mean to men. Or like being hurtful towards them, or like "poor that guy for what you are like to him'. I didn't understand how that could possibly come to a conclusion, because I didn't feel like I had any sort of control over any of those situations. I felt like I couldn't do anything. 
This loss of control also contributed to Fay blaming herself for the way the Residential Assistant was acting toward her, but this self-blame has since subsided. Self-blame is a common impact for victims/survivors of sexual violence (Kelly, 1988; Hayes-Smith \& Levett, 2010; Walsh et al., 2010; Basile \& Smith, 2011), so it is not surprising that this was reported by one of the participants in this research. Fay's identification of the power imbalance between herself and her Residential Assistant highlights the ways that differences in power due to differences in social hierarchies can exacerbate feeling a loss of agency and internalisation of self-blame.

Indi also described the impacts that experiencing unwanted sexual comments from a resident at the hall had on her identity and "the way you feel about yourself." Bianca's experience altered the way she personally felt about others. She disclosed that she had previously been a victim/survivor of rape during high school, and that history, combined with the impact of living in the hall environment, reinforced her difficulties with trust and her views of men in general:

I've had too many - in high school I was a victim of rape, and that has taught me the very hardest way it could, not to trust people... I don't have a great outlook on men, in general. I don't tend to stay away from men as such but it takes me a very long time to trust someone who is male. And that goes back to what happened to me at high school as well. But this just reinforced it for me.

In Bianca's case, her experience of revictimisation at university reinforced previous feelings of trauma from her high school rape, thus exacerbating the impact that such feelings had on her sense of self. Some scholars have suggested that one of the most devastating consequences of sexual violence is the increased risk it poses for women in terms of experiencing such violence in the future (Katz et al., 2010; Calhoun et al., 2012). There is an emerging body of literature which documents the risk of revictimisation for university students in the university context (Crawford et al., 2008; Fisher et al., 2009; Krebs et al., 2009), yet no research exists in this area in New Zealand.

\section{Perceptions of Safety}

Experiencing sexual violence can upset the perceptions of safety that a victim/survivor has, and can significantly impact their ability to feel safe in the 
future (Culbertson, Vik \& Kooiman, 2001; Sampson, 2002). Many victims/survivors report intense fear following sexual victimisation (Yuan et al., 2006; Calhoun et al., 2012). This fear is likely to be heightened in the presence of a perpetrator (Kelly, 1988). This was very much the case for Indi, who felt safe "for the most part" in her hall in first year, however she continued to feel uncomfortable in that environment. In her second hall, she said "it was just the same deal really where I just felt terribly uncomfortable when he was around at our flat. Other than that it was fine, I felt safe." Further, Sampson (2002) argues that the fear of running into a perpetrator in a dormitory can encourage women to drop out of university altogether. This was very much a reality for Fay, who reported that she "didn't wanna be [at the hall] anymore... mainly coz of the sexual reasons." The very nature of the hall environment exacerbates feelings of fear, as it is difficult in such a public and shared space to remain hidden from a perpetrator. This prevents women's equal enjoyment of the university experience (Day, 1994; Kelly \& Torres, 2006) and is evidence of the importance of ensuring women's safety in university halls.

All of the women commented on their perceptions of safety within the university hall. Some of the women felt unsafe, whilst others felt that the hall environment did not have such a substantial impact on their feelings of safety. Dana reflected on safety concerns within her hall, which were influenced by the ways she felt that her disclosure had been responded to and handled:

I dunno, it's really isolating and it's pretty terrifying as well to know that the place where you're living, the place where they over and over and over again say it's a home away from home, you'll feel safe. No you're not... you just feel completely unsafe.

Despite some of the women feeling relatively safe in their hall, Indi remembers that the ongoing behaviour and harassment from a fellow resident meant that "it got to a point later in the year that I wanted to lock my bedroom door." Whilst locking a door may seem like an obvious decision in a shared living space such as a hall, some research suggests that the notions of halls as 'safe' places may encourage students not to take routine measures, such as locking their door (Fisher et al., 2009). This demonstrates the influence that stereotypes of universities as safe havens can have on the reality of living in a university-run environment. 
The desire to alter the physical environment to ensure personal safety was described by Dana, who recalled blocking her door like "jenga" so that in the event of unwanted entry, she would be awoken by the noise:

I would barricade my door with things [laughter]. I had it down to a science, it was like jenga. But like I would have everything perfectly aligned so that if at any point, the door was knocked by anybody, stuff would fall over and make a lot of noise and wake me up.

Dana notes that this continues to be a consequence of her hall victimisation today:

And yeah I'm super aware of where windows are at certain things, I'm suspicious of people and all that... like if I was in the bathroom and then I saw someone walk past the window, I'd just kinda [duck]. Okay threat passed, carry on, can rinse the conditioner out of your eyes now... And I would like inspect bathrooms. And I still do it now. Now its just subconscious.

This finding highlights how these women's perceptions of safety manifested in physical attempts to reclaim that safety, and these attempts remain present today for Dana. Bianca reclaimed safety in a different way, by removing herself from the situation and socially withdrawing. She commented that she "stuck to myself in my room coz I just didn't want to be a part of it. I didn't want to be a victim of it, I didn't want to see it." Fay did the same following her sexual history becoming public, and found that she avoided the dining areas and withdrew from her social circles due to the ostracism she was feeling in these public spaces:

And it got to the point where I didn't want to leave my room because people were like genuinely talking about me... I mean I didn't go down to the dining hall so I ate like Mi Goreng for like months. Well not months, maybe like two weeks, that was an exaggeration... I kept my door closed and I'd just stay in my room all the time... it was just a really miserable time.

Withdrawal following an incident of sexual violence is frequently referred to in academic literature (Basile \& Smith, 2011; Calhoun et al., 2012). However, the very public nature of the shared living spaces that halls provide may actually prompt victim withdrawal and a desire to be invisible. Conversely, the social nature of the hall environment could assist the identification of students experiencing difficulties by friends or hall management. This raises important questions about the availability of support services for students experiencing 
difficulties and the need to ensure that such services are readily accessible for students to utilise.

\section{Anxiety}

Anxiety is a common postvictimisation experience for victims/survivors of sexual violence, with high numbers developing anxiety (Campbell, Dworkin \& Cabral, 2009; Basile \& Smith, 2011; Campbell \& Townsend, 2011; Calhoun et al., 2012; Jordan et al., 2014). Two out of four of the women who took part in this research explicitly identified some level of anxiety in their responses postvictimisation, however all of the women commented on ways that their experiences contributed to feelings of anxiety. Fay explicitly described the anxiety she felt knowing that a Residential Assistant at her hall was passing on information about her sexual activity to her cousin. This fear can be viewed in conjunction with Fay's anxieties about her family finding out about what happened at the hall, which will be discussed later in Chapter Six. As halls are unique microcosms of the wider university population, the spreading of such gossip can move quickly and the boundaries regarding who may find out are expanded. The large-scale spread of sexual gossip, therefore, is a significant factor in developing feelings of anxiety in halls of residence.

Fay continues to experience "really bad anxiety" in her attendance at law school. Fay's anxiety at attending law school stems from a range of incidents which took place at her hall. At one point, a sexual act that she consented to became public knowledge in the hall. Unfortunately, a number of residents approached a law tutor and suggested that using that incident should be the problem question for the next study tutorial at the hall. This meant that they discussed her intimate sexual history “...for the next hour... in like a legal sense. So it was just like really awful! It was so awful!" These findings show that the small communities within the hall, as well as the closeness of the law school cohort, exacerbated the impacts of her sexual history becoming common knowledge, thus affecting both her hall experience and her wider university study.

Indi recalled feeling anxious about a problematic resident from her hall in first year who ended up spending a lot of time at her hall in second year. She 
remembered discussing her unease at his presence with other girls in the flat, who shared similar anxieties as "they both said, and I had the same thought, that the first thing that went through their mind was him going through our underwear drawer or something." Further, Indi recalled putting the thought of this out of her mind at the time, but the shared anxieties that the girls had meant Indi felt that her concerns were "a bit more legitimate" and "an actual concern". Whilst Indi's flatmates shared her concerns, the outcome of her concerns seeming legitimate may have been different if her flatmates had dismissed her concerns. Previous research suggests that negative responses from others in the event of a disclosure, or in Indi's case an airing of concerns, can inhibit victims/survivors' ability to disclose in the future (Orchowski \& Gidcyz, 2012), and this finding highlights the importance of victims/survivors feeling that their concerns are legitimate and validated.

\section{Alcohol and Other Substance Use}

Alcohol and other substance use is a common coping mechanism for some victims/survivors in the aftermath of experiencing sexual violence (Campbell et al., 2009; Campbell \& Townsend, 2011; Calhoun et al., 2012). This is potentially problematic given the impact this can have on women's overall physical and mental health, as well as the potential for increased risk of victimisation (Abbey et al., 1998; Abbey, 2002; Adams-Curtis \& Forbes, 2004). Only one participant in this research commented on her misuse of alcohol postvictimisation. Fay reports that she "started getting really, really, really, really drunk a lot of the time" and this impacted on how she would have appeared to others. Fay indicates that she "would've been awful. I was drunk a lot and I was like crying all the time and like generally not being a very fun friend to have." Interestingly, Dana recalls management at her hall expressing concern about her having an $\mathrm{RTD}^{16}$ in her wardrobe, but she felt that trivial events were followed up at her hall and serious incidents were not reprimanded:

Why does it matter? I found myself thinking that. Why does it matter if I get myself in trouble for drinking? What's gonna happen to me? A guy that commits rape is welcomed back the next year. What's your threshold here, at what point do you kick someone out?

\footnotetext{
${ }^{16}$ An RTD is a ready-to-drink, pre-mixed spirit.
} 
This finding highlights the double-standard in her hall management's attitude toward alcohol consumption versus sexual violence, and the impact that such attitudes held by management can have on residents' decisions to participate in certain behaviours. Further, resident knowledge about previous responses to behaviours displayed by others in the hall may work to encourage or discourage participation in particular behaviours. This may have implications for the way that hall management responds to resident conduct, and emphasises the importance of getting things right the first time to ensure that inadequate responses do not become an expectation by students, or a standard by management.

\section{Difficulties sleeping}

Difficulties sleeping are a commonly reported sequelae following sexual violence victimisation (Yuan et al., 2006; Jordan et al., 2014). Indi reported that she was "losing a lot of sleep", and became particularly concerned about the ways the resident who had harassed her was continuing to harass her friend at the hall she previously lived in:

I became very emotionally stressed, especially hearing about the situation with my friend who was back at [name of hall]. Like, I was losing a lot of sleep... Like I would get up in the morning and then if I had a class at yknow 10 or 12, I would get up, get ready and then I would have like a powernap before class in the morning.

Indi's reflection on her difficulties sleeping identified that it was not only her own experience troubling her, but the stress of hearing about her friend experiencing similar behaviour triggered a similar emotional response. As the majority of university women disclose to friends (an area that will be discussed in more detail in Chapter Six), this finding highlights the importance of not only ensuring that victims/survivors are equipped with appropriate support following victimisation, but also that students generally are equipped with knowledge about how to manage disclosures from their peers.

\section{Flashbacks and Panic Attacks}

Flashbacks are vivid recollections of memories, in this instance trauma, that are often accompanied by panic attack symptoms and dream disturbances (Mcnally, 2005). Re-experiencing of the event, resulting in "intense psychological distress 
at exposure to internal or external cues that symbolize or resemble an aspect of the traumatic event" (Johnson, 2009: 6) is a frequent response to trauma reported by victims/survivors of sexual violence (Basile \& Smith, 2011). Kelly (1988) discusses flashbacks in her qualitative work with victims/survivors of sexual violence, noting that the most common trigger for a flashback is something specific that reminds women of the assault, such as a location or a kind of movement. Dana described a flashback she experienced vividly, which was triggered by a movement, and her associated physical response to it:

Like coz the bathroom downstairs is where it happened. And there was a bathroom upstairs. And at one point I was visiting friends who lived in that, in the second floor. And I was in that top bathroom using the toilet. And then under the door - you couldn't see under the door, you couldn't see through the door at all and I knew it - but I saw a shadow move past. And for some reason I just freaked out and like wouldn't leave. And then kept trying to convince myself that I could leave and kept saying like 'right no, you'll go out and then you'll go against the wall and then you'll have like all your periphery'. It was like a tactical mission to get down the hall and I knew that it was completely irrational but I couldn't shake it.

Clearly, Dana's experience of feeling like somebody was watching her triggered a significant emotional response, and her description of a tactile mission to get away from the situation is a resounding one that signifies the impact that an experience like her's can have on women.

Dana described how she tried to avoid particular places that reminded her of the event, which had consequences for her in her living environment. She recalls getting "in trouble for using upstairs bathroom or bathrooms from other houses" as well as being instructed by hall management to use the bathroom where the incident took place. Having to use that bathroom triggered panic attacks:

Not necessarily sure if its panic attacks, but I get super upset and panicky whenever I was in that building... I said that I was having panic attacks going back into that room. And throughout the year I was having panic attacks going into that building.

Experiencing panic attacks following sexual victimisation is a common impact for many victims (Campbell et al., 2009; Palmer \& Parekh, 2014). Dana's description of experiencing panic attacks, as well as the response by management highlights how the directives of hall management exacerbated the impacts she felt. The negative impacts on overall wellbeing for victims/survivors 
of sexual violence are cumulative and stem from multiple different factors (Campbell et al., 2009). The way that management responded to Dana's experience and their directives to continue using the same bathroom where the incident occurred are problematic and indicate a real need for thorough education about the impacts of sexual violence on victims/survivors, which will be explored later in this thesis.

\section{Social Impacts}

Experiencing sexual violence can impact victims/survivors on many levels, and the impact of sexual violence on a social level has been a research focus over the years. The social consequences of sexual violence can be significant and severe (Garcia-Moreno et al., 2012), therefore exploring the social impact of sexual violence among university hall populations is crucial given the ongoing, daily interaction between residents which epitomises the hall environment (Fisher \& Wilkes, 2003).

\section{Difficulties in Interpersonal Relationships}

Evidence suggests that experiencing sexual violence can have substantial impacts on the management of interpersonal relationships (Basile \& Smith, 2011; Boyd, 2011). Whilst all except one of the women described being in, or on a break from, an intimate relationship at the time of their experience, only one participant reported that her intimate relationship became affected as a result. Fay had been on a break from her relationship at the time everything happened at her hall, and the burden carried from that was a large one:

My relationship with my boyfriend broke up and I felt so guilty all the time and I was like completely obsessed with - because we kinda got back together after that - but then I couldn't do it because I was so obsessed with making sure that he never knew. He still doesn't know and he's my best friend now. He can never find out because it'll destroy him.

In many ways, the guilt that Fay experienced following the incident - in conjunction with the ongoing sexual harassment - contributed to the overall impact of the experience. However, Fay also describes a time when she had told a group of her friends "how much everything hurt" and how she experienced confusion surrounding where, or to whom, she should place her affections. This 
confusion had further impacts, in that she ended up getting attached to the guy who told everyone about their sexual history:

And then I ended up actually getting really attached to the guy... And then he wasn't actually very nice to me... he seemed to think I was slutty for it. While having sex with me for the next few months... And like after he like ended our thing, he came over once and we had sex and then I was like to him 'maybe you should probably not have sex with girls if you don't really like them' and he was like 'well you shouldn't have sex with people if you know they really don't like you', and that was like while he was in me"

Fay explained that this experience made her feel "pretty shit. I felt like nothing because I was having sex. And like it's not how 18 year old girls should feel, like at all. So I found that really hard." Fay's involvement with the resident who had told everybody initially about their sexual activity, and then subsequently treated her badly, is telling because Fay also reports that her friends were unable to understand why she went back to him either, leaving her with no support. Fay recalls her relationships with her friends became increasingly strained once her sexual history became public knowledge in her hall:

And then my relationship with my friends started kind of falling apart because they'd never had sex before, some of them, and the ones that had hadn't sort of had like this horrible juxtaposition of very intense wonderful relationship but kind of falling out of love with them as you like go to university and aren't in high school anymore, with like a really socially shameful activity which a lot of people found out about. So they couldn't deal with it. And then so my relationship with them sort of was really, really strained, also.

Indi also remembered feeling annoyed at her friends who she believed were continually inviting the man over who had been harassing her, which led her to withdrawing herself from social situations:

Especially coz I thought that they were inviting him over. I just felt like a grumpy bitch sitting in my room with the door closed. Like, sitting there thinking 'I wish he would fuck off'. And then, yknow, I was annoyed at them because I thought they were inviting him over but then I kinda realised he was sort of inviting himself over. Causing all the problems.

As sexual violence often impacts on intimate relationships, unsurprisingly then, sexual violence also impacts on many victims/survivors' ability to maintain stable friendships postvictimisation, thus having a significant impact in the social domain (Jordan et al., 2014). For both Fay and Indi, their experiences of victimisation had a direct association with the difficulties they were 
encountering socially. Both women's stories indicate the importance of the delivery of education about how to manage disclosures and how to be a good friend (Walsh et al., 2010).

\section{Academic Impacts}

Investigation of the impact that experiencing sexual violence can have on academic outcomes for university students is an area that has been largely overlooked by researchers to date, and the volume of literature in this field remains somewhat limited (Ottens, 2001). The conceptualisation of victimisation and associated impacts among university students is well established, however the lack of research surrounding the connections between academic performance and victimisation leaves out "a major domain in the lives of female students" (Jordan et al., 2014: 193) and is thus an area that requires dedicated research attention.

Littleton (2014) hypothesises that it is "likely" that experiencing violence will have a negative impact on student performance. Wright and Bonita (2012: 195) agree, and elaborate in their discussion of the consequences of sexual harassment, stating that "female victims suffer more significant educational consequences of harassment, in that they are more likely to find it hard to pay attention or concentrate while in class." Further, Jordan et al. (2014) argue that a woman suffering sequelae in the aftermath of experiencing sexual violence may experience cognitive impairments, and depression and anxiety may "decrease her ability to engage with other students due to social anxiety, shame or embarrassment." (p. 197). This particular finding came through in an interview for this research. Fay commented on the impact that experiencing sexual violence in her hall had on her academic performance, and her ability to concentrate on a particular paper as the perpetrator was still in that class:

My grades got pretty shit for a bit. I associated that experience with the class that he was in, which was a really difficult paper. So I found it really hard to actually study for that paper.

Further, Fay recalls the impact that her experience had on her ability to attend university, and she "just wouldn't leave my room. I kept my door closed and I'd just stay in my room all the time... And then I kinda got to the point where I like started going to uni again." 
The investigation of impacts on academic performance is important, particularly in the context of university halls, as students living in halls are in near constant contact with other residents, as halls of residences are by nature social environments (Krebs et al., 2009). As the majority of sexual violence incidents are perpetrated by somebody known to them (Sampson, 2002; Fisher et al., 2009), and often in the same residence (Day, 1994; Wasserman, 2003; Fisher et al., 2009), this raises questions about the difficulties that students in halls may face in terms of their educational outcomes, due to ongoing contact with perpetrators.

While Fay's experience indicates that the academic impacts for her were predominantly short-term, as previously discussed, she continues to experience anxiety in attending law school. Fay's comment about not enjoying attending law school, and in a sense forcing herself to attend, raises questions about wider issues regarding student retention. Lindquist et al. (2013: 2441) discuss the actions that a victim/survivor may take following experiencing sexual violence, and argue that "survivors might drop classes, transfer to another school, or move." Jordan et al. (2014) argue that sexual violence as a risk factor for dropping out of particular classes, or the university as a whole, is notably absent in the literature on student retention. Therefore, it would be beneficial for more research to be conducted that explores the links between sexual violence and academic impacts, including the links between sexual violence and student retention.

\section{Summary}

This chapter has documented the extensive impacts and consequences that participants experienced in the aftermath of experiencing sexual violence in their university hall. It also highlights the differential consequences that experiencing sexual violence can have on women, and the cumulative effects of sexual victimisation. The women reported numerous impacts that affected their self-schemas, their psychological and social functioning, as well as reporting impacts on educational performance and achievement. Whilst the small sample size involved with this research impacts on the generalizability of the data, these impacts are both similar and unique to the existing literature on women's impacts from sexual victimisation. The impacts that the women reported are 
important due to the little documented information of the impacts of sexual victimisation among New Zealand university women, particularly those living in unique environments such as halls of residences. This chapter has also identified a number of areas that warrant further research, or avenues for potential delivery of education in the context of response and prevention efforts to minimise further trauma for women who are victims/survivors of sexual violence. 


\section{Disclosing Sexual Victimisation and Seeking Help: In Whom Do University Women Confide?}

"No, I never talked because I was taught at high school snitches get stitches"(Bianca)

Disclosing sexual violence is a difficult issue, and the decision to disclose can have unexpected outcomes for victims/survivors (Walsh et al., 2010). This uncertainty means that the majority of sexually violent incidents sit under a cloak of silence and do not come to the attention of authorities (Fisher et al., 2009; Miller et al., 2011; Wolitzky-Taylor et al., 2011). Research with university women has consistently shown that they are unlikely to report to the police, however many women will disclose informally to those around them (Krebs et al., 2007; Fisher et al., 2009; Edwards, Dardis \& Gidycz, 2012). With this in mind, this chapter aims to understand who the women interviewed for this research confided in, and why they chose such people as confidantes. This chapter firstly explores participants' experiences of disclosure to formal sources, in this case, the police. Next, this chapter explores women's experiences of disclosure to other supports such as hall management, friends, family and intimate partners. This chapter then looks at the help-seeking actions made by participants. Although the sample size in this research is small, this chapter fills a unique literature gap in understandings of sexual violence disclosure and help-seeking actions by New Zealand university hall residents. 


\section{Formal Disclosures}

\section{Underreporting of Sexual Violence to Police}

The underreporting of sexual violence is a chronic issue, with the majority of sexually violent incidents occurring beneath the radar of formal authorities (Fisher et al., 2009; Miller et al., 2011; Wolitzky-Taylor et al., 2011). In fact, it is estimated that only one in every nine incidents of sexual violence is reported to New Zealand authorities (Ministry of Women's Affairs, 2009). International research highlights that university women are not exempt from low reporting rates, and underreporting to formal authorities has been identified as a pervasive issue (Krebs et al., 2007; Fisher et al., 2009; National Union of Students, 2010; Walsh et al., 2010; Branch, Hayes-Smith \& Richards, 2011; Zinzow \& Thompson, 2011).

Understanding the complexities behind reporting sexual violence is crucial, as identifying particular barriers to disclosure means that solutions can be developed to destabilise them (Zinzow \& Thompson, 2011). A barrier has been defined as:

any factor that serves as an impediment to disclosure, reporting, or help seeking and that makes it less likely that a survivor will tell someone else about his or her victimization or seek formal services for help in the aftermath of the victimization (Walsh et al., 2010: 136-137).

Victims of sexual violence often remain silent due to the "myriad of obstacles they must face in reporting such crimes" (Branch et al., 2011: 56). Barriers to disclosure include embarrassment, fear, self-blame and shame, among many others (Fisher et al., 2009; Walsh et al., 2010; Daigle, 2012). Further, many victims/survivors report fear of negative social reactions, whether that be from friends or formal authorities (Campbell, Wasco, Ahrens, Sefl \& Barnes, 2001; Ullman, 2010). Numerous researchers have investigated and commented on women rape victims' experiences with the police, with negative treatment by police being a common experience (Jordan, 2004; Page, 2010). Such 'secondary victimisation' continues to inhibit victims' decisions to come forward and disclose their experience, thus contributing to widespread underreporting (Campbell \& Raja, 1999). Widespread underreporting is a major barrier to criminal prosecution, apprehensions and the ability to connect victims with available and existing resources (Walsh et al., 2010; Zinzow \& Thompson, 2011), 
so research into underreporting and initiatives to encourage reporting must be ongoing.

\section{Participants' Experiences with Disclosure to Police}

Although the sample size for this research is small, underreporting of sexual violence by this sample correlates with the literature on sexual victimisation disclosure among university populations (Fisher et al., 2009; Walsh et al., 2010; Zinzow \& Thompson, 2011). Only one participant in this research formally reported her disclosure to the Police. Dana told her friend, who also happened to be a Residential Assistant, that she had noticed someone filming her in the shower. The Residential Assistant called the Police on her behalf:

I went and told my friend who was on duty about it and he called the Police. Police filed a complaint and everything. And then it was noted in a like red exercise book that was kept in the office... I didn't think what happened to me was a crime at all. I was really surprised the Police were called or anything.

Dana's difficulty in identifying what happened to her as a crime is a common theme among university students who have experienced sexual violence. Fisher et al. (2009) found that of students reporting attempted or completed rape, uncertainty about whether what happened was a crime or whether harm was intended was the third most common reason stated for not reporting sexual victimisation. Further, data from Zinzow and Thompson's (2011) study of 127 female undergraduate sexual assault victims affirms this, as "not serious, not a crime" (p. 718) was the second most common reason for not reporting by university women. Such findings indicate that identifying an incident as sexually violent or a serious crime is difficult, so this opens up potential avenues for education about what constitutes sexual violence within university populations.

Dana made her statement on her own without support, which is an issue identified by Key Informant 1, who noted that "if a young one is going through making a formal statement, then support for them is often not there." However, for Dana, not having the support of anyone from the hall or hall management was actually a preference. Dana had a positive experience with making a statement to Police:

I was quite happy, it was really cathartic being able to say things to Police. Just to have somebody. I remember the first thing they did when 
they sat down opposite me. The first thing she said was this is a crime and we know it. And at the point I was like oh my god thank god! I'm not crazy!

Dana's need for validation and to be believed postassault is a common desire for victims/survivors and can be important in the road to recovery (Walsh et al., 2010). Dana also remarked that having her experience validated left her feeling "chuffed" and that the Police "did no wrong in my eyes. Nothing that they did could ever be wrong." Key informant 1 also expressed praise for the Police and the way they dealt with student victims/survivors, and identified ways that the Police actively tried to make it easier for young women to disclose:

[The Police] are being absolutely fantastic and my working with them... especially around rape situations and things like that with young girls on campus, that they will meet with them on the university and not have to take them down to the police station and things like that, has been superb.

Although this sample is too small to make generalisations about hall residents' and students' disclosures to Police, it is promising to see the Police have worked well with the students discussed here. However, it is important to note that not all women who report sexual victimisation to the Police have good experiences with them, so her experience highlights the limitations that an individualised criminal justice system response elicits (Jordan, 2004; Phipps \& Smith, 2012). In fact, some university women actually regret telling the Police because of the way they are treated (Krebs et al., 2007). Nevertheless, such positive experiences with the Police are encouraging and more research on New Zealand students' disclosures to Police would be a valuable area of inquiry.

\section{Disclosures to Others}

Although few students disclose formally to Police, a significant number disclose to less formal supports around them. Previous US research indicates that over two thirds of students have disclosed to at least one other person (Krebs et al., 2007; Fisher et al., 2009), most commonly to friends and family (Banyard, Moynihan, Walsh, Cohn \& Ward., 2010; Edwards et al., 2012). Disclosures can be beneficial for some victims, as the act of disclosure can

serve as a first step toward connecting victims to sources of support, medical care, and mental health services, and may facilitate formal reporting to the authorities. Connection to services is particularly important when considering the low service utilization rates, especially 
mental health services, by sexual assault victims. Further, disclosure can also result in the rape victim receiving emotional support (Paul et al., 2013: 487).

All of the women interviewed disclosed to informal supports either at the time of the incident, or later on during their university studies. Participants' experiences of disclosure will now be explored in turn.

\section{Hall Management}

All halls at Victoria University have an internal management structure, which usually consists of Residential Assistants, Residential Life Managers and Hall Managers. All of the women reflected on their hall management, and commented on the ways in management would have responded to a disclosure, or the ways management actually responded when incidents arose. Indi felt that disclosure to management would not have yielded appropriate outcomes:

... I wanna say that he would've, it would've been like a slap on the wrist with a wet bus ticket, yknow? <laughs> I don't think it would've gone particularly far. She might've had a word to him, and he might've said 'mmm people can't take a joke, uh' and that would've been about it.

Dana recalled that management were aware of what was happening with a particular resident, as it was happening in public places, but nothing was being done about it. In turn, this contributed to her lack of disclosure to management as she internalised their attitudes:

Hall management would just laugh it off and be like 'ah boys will be boys' or like 'well maybe you should try not to like dress so tight and then he won't notice you or something'. And then during 2011 I felt kind of uncomfortable about it, but I didn't do anything, like I didn't follow it up at all because I brought into that whole 'boys will be boys'.

Not only were the attitudes by management identified as barriers to disclosure, Fay also identified two other barriers which discouraged reporting to those in managerial positions:

No, I should have officially complained because that man was a tutor for the university... I should've lodged a formal complaint... I didn't realise what he was doing was so wrong. I also didn't know how to lodge a formal complaint.

Fay's minimisation of the seriousness of what happened, in conjunction with not realising what happened was a crime, echoes findings from other research (Fisher et al., 2009; National Union of Students, 2010). Fisher et al. (2009) 
found that in their National College Women's Sexual Victimisation Study, 20 percent of attempted, or completed, rape victims cited not knowing how to report victimisation as a reason for not reporting formally to Police. This lack of knowledge about how to report is echoed in the hall environment, as Fay was unaware of how to report what was happening to people in the hall. Neidig (2009) argues that significant policy concerns exist around vague institutional reporting requirements on campus in the US. Karjane et al. (2005) suggest that to combat such ambiguity, university policies on sexual assault should be accessible and widely distributed to university students. Dana commented that an open and transparent sexual assault policy would have prevented a lot of what happened in her hall, which suggests that such policies may not be available for students currently. I would argue that vague institutional reporting requirements may exist in university halls, which identifies an avenue for potential thinking about how to clarify the process for reporting in halls to encourage increased transparency and understanding about the process for reporting.

Dana's disclosure to a Residential Assistant meant that management were later informed about the incident and became involved in the investigation process once they returned from holiday:

A few days later, Head Management came back. And they apologised and seemed like they were really interested in finding the person but they were really hesitant about involving the Police. And they seemed kind of cagey that the Police were called in the first place... the Police said that they would contact the hall to pursue it further in a few weeks' time when they'd kinda processed it and ordered their crimes... And I never got the call back... And then a few days later I was called into the office by myself and I was told that in fact the Police had called pretty soon after, like within a few days after, and they didn't feel the need to pass it on because they didn't think it was necessary.

For Dana, the lack of involvement in her case by management, which continued for a significant period, contributed to a number of negative personal impacts that she experienced. Dana sought validation from management, just as she had sought - and received - from the Police. Instead, she ended up searching online for answers for how she was feeling postassault:

It wasn't my crisis they were looking out for, it was their crisis. It was their reputation and a bunch of adults fluffing round wondering what's 
going to happen to the hall's reputation... In this whole two months [management have] given me less than what, like one paragraph of a webpage has said in saying it's not your fault.

The lack of validation from management of her experience meant that Dana began to tell others not to pursue such support through similar channels:

And moreover like I found myself saying quite a few times, like if something happens, don't go to hall management about it. Go to the university about it and go to the Police. Get a Police complaint lodged. And just get it on record that you're uncomfortable. But there's no point trying to go to hall management because they will make your life miserable.

This finding highlights the ways that particular responses to disclosures can result in a standard practice of continued silence. In turn, such continued silence can give false indications to university administrators regarding the extent of sexual violence within their residences (Ottens, 2001; Argiero et al., 2010). This can mean that sexual violence is ignored and does not receive the attention that it deserves. Whilst Dana's experience is unable to be compared to others in this research, nor is it likely to be generalizable to other's experiences, it does highlight the importance of ensuring that a seamless and appropriate response mechanism exists within halls of residence to ensure that students feel comfortable speaking with management during times of crisis.

\section{Residential Assistants}

Novak (2008) contends that Residential Assistants are most likely to be the first responders to situations of crisis within hall environments. However, findings from the National College Women's Sexual Victimisation Study dispute this, as victims very "rarely contacted university officials, hall advisors, or help services" (Fisher et al., 2009: 151). As previously mentioned, one participant disclosed to a Residential Assistant, who later informed the Police and hall management. However, one other participant discussed her Residential Assistant at the hall, and how her lack of being 'involved' meant that she did not turn to her for help:

My RA was actually extremely lovely, she was a lovely, lovely woman. But she wasn't particularly involved. Which I kinda liked... like after what happened in the second half of the year, I think I would've wanted her to be more involved... so I could've felt like I wanted to go talk to her (Fay) 
Fay elaborated on what she felt should be more of a focus for students who become Residential Assistants, and what characteristics would be useful for people in that role:

... people that would actually be good counsellors. Because I think when you're with a bunch of 18 year olds like who have just moved out of home, there's gonna be so many issues, like issues with anxiety, like it's a huge change. It's not even as if you're going into a flat which could be comparable to a family. This is like the most artificial situation ever.

That said, all Residential Assistants at Victoria now receive some training on sexual violence, which is in its third year. Key informant 6 reflected on the training that Residential Assistants receive at Victoria:

I think the training has got better and better each year as it becomes something that, I'll use the term celebrated because we're doing something that we believe in. And certainly the [Residential Assistants] are 100 percent in terms of buying into it, there's not sort of push back or denial or hey look that's too difficult to talk about. There's definitely a culture of wanting to engage in that topic.

Whilst it is unfeasible that all residents would feel comfortable speaking with their Residential Assistant about sexual violence, Residential Assistants must be equipped to respond in the event of disclosure. The ongoing training of Residential Assistants to ensure their active involvement in the welfare needs of students within their halls is an important area and it is promising to see such initiatives being implemented at Victoria University.

\section{Friends}

The majority of disclosures made by university women are to their friends, peers or people they live in close quarters with, such as roommates (Fisher et al., 2009; Walsh et al., 2010). This is unsurprising as the majority of people that students in halls will be in close contact with are their peers (Orchowski \& Gidycz, 2012). All of the women interviewed for this research disclosed to their friends or those around them, either at the time, or more recently:

What was happening at the hall, I talked about with one of the guys I hung out with at uni every now and again, and he was just like 'it's guys being guys'. I was just like you don't act like that towards me, why should they?... I did talk to my friends about it a little bit (Bianca)

I talked to all my flatmates and my other group of friends about it (Indi) 
Bianca reported that discussions she had with her friends encouraged her not to follow things up with hall management:

At the time I didn't think it was that big a deal...but most [friends] knew that through high school it was pretty fucking bad with guys. And they knew that I could take care of myself. Um, so we kind of as a team, kind of just left it. Coz we knew if it was going to get bad, I would say something... I'm more a sort it out myself, go talk to a counsellor in a week or two kinda girl than I am to go running to whoever's around.

The idea of being able to 'handle it myself' is a known barrier to disclosure to the Police for university women (Fisher et al., 2009); however this notion of dealing with it herself is also relevant in terms of disclosure to hall management. In this sense, it is a barrier to disclosure to both formal and less formal supports.

Fay was the only participant that did not disclose informally to her friends at the time of the incident; however this is largely because "everyone knew" about what had happened. Fay's sexual history becoming public resulted in her friends taking action to confront her, sparking an "interfriendtion ${ }^{17}$." Fay's overview of the interfriendtion, which happened twice, highlights the exact type of negative responses that many victims/survivors fear (Edwards, Dardis, Sylaska \& Gidycz., 2014):

They gave me an interfriendtion, which was like the worst thing that ever happened. No that's exaggerating, it was a pretty shitty thing to happen. It was like all my friends just ganged up on me and said all these things they didn't like that I did... they all came in and they read off bits of paper all the stuff they hated about me and then they were like 'we're concerned'... And then they left... when the group of people that you trust tell you everything they don't like about you and tell you why it's problematic and then tell you to come and stand up in front of them in a room and talk to them about it, it's being on trial essentially. Like trial by friendship.

The public knowledge of the incident meant that there was no need to disclose, however the negative consequences that victims often fear they will receive upon disclosing eventuated anyway. Fay recently disclosed to a group of friends about what had happened with the law tutorial held at the hall, where her sexual history became the subject of a communal problem question:

\footnotetext{
${ }^{17}$ An interfriendtion is a term that describes an intervention being given to a friend who is not behaving how they should be or is continually making mistakes. The term has been referred to on 'The Real' television show http://thereal.com/videos/o-9q8tcj12/, in the movie Sex and the City 2 and on the television show How I Met Your Mother.
} 
At the time I didn't think it was an issue so I didn't tell anyone... maybe two months ago actually, I was telling some of my friends who didn't have a hall experience about it and then they were like 'that's not a thing that's normal.

Not being able to recognise an incident as sexually violent, or minimising the seriousness of the incident, is another well-known barrier to disclosure for university women (Fisher et al., 2009). In this vein, this finding correlates with existing research in this field around hall residents' disclosures of sexual violence.

\section{Family}

Whilst some scholars suggest that victims are most likely to disclose to friends and family, recent research with university students indicates that reporting to family is actually rather low. Orchowski and Gidycz (2012) describe low rates of sexual assault disclosure among university students as unsurprising, as "women attending residential institutions of education spend vastly more time with their peers, compared with their parents" (p. 278). Data from the National College Women's Sexual Victimisation Study suggests that only 10 percent of university women discussed their victimisation with a family member (Fisher et al., 2009). Whilst the sample in this research is not generalizable, only one participant disclosed to her family, and one other commented on her inability to tell them at all. Dana was selective with who she told as she feared what the consequences would be if her grandparents found out:

I only told my Mum because my grandparents are super protective and I knew that they'd just lose it and insist that I move back home. She said that it was obviously super bad and that she felt sorry for me and that. She was all good.

The fear of negative social reactions from others is a commonly discussed theme regarding disclosures of sexual violence (Orchowski \& Gidycz, 2012; Edwards et al., 2014). Further, not wanting family to find out about what happened is frequently discussed when referring to why official disclosures to Police are not made (Fisher et al., 2009; National Union of Students, 2010). However an overlooked theme, especially with students living in halls, relates to the impact that a disclosure may have on their residential status. Branch et al. (2009) summarise why university women may not want to disclose to their families: 
Many college students, especially those between the ages of 18 and 24, are testing their independence from their parents, living away from home for the first time. As such, college women may not want to disclose sexual assault or IPV victimization to their families because it may make them feel like they have failed in taking care of themselves away from home ( $\mathrm{p}$. 56).

I would add to this that students living in halls of residence may fear that this 'failure to be independent' means that they need to move back home where existing parental safeguards exist and students can be protected. Whilst it is not possible to conclusively say whether Dana feared failure as such, her concerns about being forced to move home are clear and may be a unique barrier to disclosure for students living in halls.

Fay felt like she could not disclose to any of her family - especially her Mum about what she was going through at the hall:

I obviously couldn't tell my family... And I couldn't tell my parents coz it's like "oh Mum [sexual history became public] and now everyone hates me". Even if I did have that kind of relationship with my Mum, where I could share that kind of thing, I wouldn't want to. I wouldn't want to put my Mum through the pain...

For Fay, the difficulty involved in telling her family about what had happened is that her family would then know that she had been involved in a "socially shameful activity", which would be painful for her Mum to hear. The fear of hurting family members by disclosing has not previously been discussed as a barrier in literature with university students so such a finding may elaborate on Orchowski and Gidycz's (2012) work about why disclosures to family are low.

\section{Intimate Partners}

There is a paucity of research regarding university women's disclosure to intimate partners. Recent research with university students found that only 26 percent of victims disclosed to a romantic partner (Walsh et al., 2010). Data from the National College Women's Sexual Victimisation Study also shows that only 8 percent of women disclosed to a "husband, boyfriend or partner" (Fisher et al., 2009: 151). In this research, two participants discussed what was happening at the hall with their intimate partners, and the responses from intimate partners were remarkably different. Bianca reflected on a discussion she had with her boyfriend about the boys she hung out with at the hall once, 
and he got "overly jealous so I just stopped talking about it with him." Conversely, Dana disclosed to her boyfriend about what happened and he came to stay with her immediately after it happened. She notes that he "came up to stay with me for two weeks after to just be there, pretty much. And he wanted to be there as well."

Conversely, Fay never felt able to tell her boyfriend. At the time, just prior to her sexual history becoming public, she had been on a 'break' with her boyfriend, so she felt guilty for having sex with someone else during that time. She got back with her boyfriend after that, but was unable to continue being in the relationship and the guilt and secrecy continues today. Fay noted that "he still doesn't know and he's my best friend now. He can never find out because it'll destroy him." Clearly, as previously discussed in relation to Fay's difficulty in disclosing to family, she experienced significant difficulty in disclosing to her intimate partner for fear of hurting him. In this sense, again, the fear of hurting others is a barrier to disclosure for university women and has not featured in previous research with university populations.

\section{Help-Seeking Utilisation}

All of the women interviewed for this research were asked about whether they had sought help from support services following their experience(s) at the hall. There is a paucity of research regarding university women's barriers to helpseeking (Walsh et al., 2010), so the findings from this research, whilst brief, make a contribution to the literature in this field. Participants' experiences of help-seeking postvictimisation will now be explored.

\section{Counselling}

University campuses often provide a "microcosm of the services available in the larger community" (Sabina \& Ho, 2014: 202). Victoria University has a Student Counselling Service available for students to utilise. The Student Counselling Service is free for all domestic and NZAid ${ }^{18}$ students, and provides quality shortterm counselling for students who require support during their studies. Demand

\footnotetext{
18 The NZAid Programme is a New Zealand Government international aid and development programme that provides scholarships to students from developing countries to study at New Zealand tertiary institutes. More information about scholarships provided by the NZAid programme can be found at http://www.aid.govt.nz/funding-and-contracts/scholarships
} 
for student counselling is high, with 1800 students seen in 2013.19 The counselling team are poised to help students disclosing sexual violence and anecdotal evidence suggests that "probably $10 \%$ is a bit of a rough ball [figure] for students that explicitly deal with [sexual violence]" (Key informant 4). Key informant 4 elaborated on why students may feel safer disclosing at a university health setting than somewhere else:

[Student Counselling Service] often are the first place that students who have suffered either childhood or teenage sexual assault will come talk about it. And some of that's within kind of a family context and some of it's within kind of a dating relationship context and some of it's within a stranger rape, sexual assault context. And that's because... [Student Counselling Service is] a slightly safer place to disclose than perhaps when you're living in a home or in the community... that's certainly an element of this is the place and a time where students are old enough to acknowledge that yknow all is not well and they should do something about this.

None of the women interviewed identified using the services provided from Victoria's Student Counselling Service specifically; however one did speak with a counsellor "at the hall". Previous research has indicated that university women are unlikely to utilise support services postassault (Nasta et al., 2005; Fisher et al., 2009) and the findings from this research support this. Importantly, however, all participants in this research were aware of the services available on campus, which indicates that information about such services is available for students.

Of all four participants, only one participant utilised counselling about what was happening at the time, and Fay notes that she was "made" to go and see the counsellor at the hall by her friends:

Yep I saw the counsellor. Um, it went pretty well, I'd never seen a counsellor before. Did a lot of crying. What I mostly talked to the counsellor about was feeling like my friends didn't have my back. I didn't really realise that like the issue with [sexual history becoming public knowledge] was a big issue until recently, like genuinely... now I can see the connections that it's had with the rest of my shit.

Fay's use of the counsellor reiterates the importance of social integration and social supports, as mentioned in Chapter Four. Further, the push to utilise such support came from her friends, which is an important finding as some research

19 Information about Victoria University's Student Health and Counselling Service can be found at http://www.victoria.ac.nz/st services/counselling/information/staff.aspx 
suggests that up to 30 percent of students receive disclosures during their time at university (Banyard et al., 2010). This raises important questions about the delivery of information about how to respond to disclosures within student populations, and the delivery of such information in halls of residences could be a worthy area of inquiry.

The barrier of handling it independently has come through in other research with university women and their help-seeking decisions (see Sabina \& Ho, 2014 for a review). Indi encountered this barrier and felt reluctant to attend counselling, primarily because she felt that she could deal with things herself:

I knew there was Student Counselling, but I've pretty much always been kinda reluctant to go to counsellors for things because I just like to be able to just have the security of knowing that I can deal with things by myself. Because you're not always going to have a situation in life where you can have, yknow, someone support you.

Conversely, Dana felt that using the counselling services at university could have implications regarding confidentiality:

And I realised it was because in my mind, if I went through uni, it was still going through the hall. Because it's a Victoria University hall of residence. How can that be separate?... why would I go to university counselling when they'll probably just pass it back to the [management].

Concerns surrounding confidentiality have been noted in previous research with student populations and their experiences with disclosure (Nasta et al., 2005; Walsh et al., 2010), so this finding supports this previous work. Further, this finding may be useful for university services in identifying ways to reiterate information about their services remaining confidential which could encourage service utilisation by students.

The minimisation of harm or seriousness is a commonly acknowledged barrier for university women in seeking help (Fisher et al., 2009; Walsh et al., 2010), and two participants identified this as a barrier for them seeking help during their time in the hall. Dana's disclosure to Police meant they provided her with a referral to Victim Support ${ }^{20}$ that she did not utilise:

${ }^{20}$ Victim Support is a community organisation in New Zealand supported by volunteers that helps people who have been hurt by crime or other forms of trauma. More information can be found from their webpage http://www.victimsupport.org.nz/ 
The Police offered me counselling but I didn't take it. I think I knew that counselling wouldn't necessarily help, but it would just take time for me to reacclimatise back down... And I didn't think it was bad enough for Victim Support. Coz yeah, in my mind I just thought they'd probably just refer me to Rape Crisis or something. That's Rape Crisis. I'm just panicky after like something minor. So that's fine, I don't qualify for that.

Fay's thoughts on accessing specialist support echoed those of Dana, stating that she did not think that she "would have qualified for like Rape Crisis or anything." This finding identifies the ways that specific language, such as the word 'rape' and the word 'crisis', may actually discourage students from seeking specialist help. This is likely because students' understandings of what counts as 'rape' or sexual violence may be vastly different and not in line with current legal definitions. A lack of clarity around this issue may require educational efforts with university students, perhaps using halls as a starting point due to the accessibility of students, to improve understandings around what constitutes sexual violence so that students may feel less apprehensive about utilising specialist support agencies.

\section{Summary}

This chapter has acknowledged the widespread underreporting of sexual victimisation to formal authorities and explored the barriers to disclosing sexual victimisation that young women living in halls face. Whilst the sample size in this research inhibits its generalisability, this research has identified that for the women in this research, most do not disclose to formal authorities such as the Police. This lack of reporting to authorities has implications for both the apprehension of offenders as well as the linking-up of victims to available support services. Whilst one woman formally disclosed - and had a very positive experience with the New Zealand Police - it is important to not lose sight of the very real, negative experiences that many women have with formal disclosure. Bearing this in mind, more research regarding students' experiences of reporting sexual violence to the Police would be beneficial.

Whilst most women do not formally disclose, all of the women in this research disclosed to a range of people: friends, family, intimate partners, hall management, Residential Assistants and counsellors. This chapter documented their experiences with disclosure to these supports, and identified a number of barriers to disclosing to hall management. These barriers were an inability to 
identify an act as criminal, minimisation of seriousness and a belief that it could be handled on their own. The lack of disclosure to hall management that this research has highlighted is problematic because it may give a false impression to hall management that sexual violence is not an issue in halls of residence, and thus does not require active attention. The identification of these barriers, and their implications, provides a basis for such literature to grow in a New Zealand context where they have not previously been identified.

Lastly, this chapter identified participants' use of help following victimisation, and identified that whilst all of the women were aware of student services at university, none utilised them. The lack of service use by participants was centred on the same barriers identified in disclosing to informal supports, such as minimisation of seriousness and a belief that it could be handled alone. However, one participant also identified confidentiality concerns involved in using university-run services. These findings also provide an insight into the barriers in a New Zealand university sample which has previously not been explored.

Overall, this chapter has identified a number of barriers to disclosure for New Zealand university hall residents and has highlighted ways in which the provision of education to university students could go some way to destabilising these barriers. 


\section{Dealing with Sexual Violence: Response and Prevention Efforts in University Halls}

"It becomes quite an overwhelming process that's very challenging and new to most people... Very difficult each time” (Key Informant 6)

Responding to sexual violence is a complex task, and as this chapter will show, there is added complexity in university hall environments as the needs of both victims and offenders must be carefully balanced. Drawing on data from both key informants and victims/survivors, this chapter examines current responses to sexual violence in university halls of residence, and identifies key avenues for change. This chapter firstly documents the lack of information and momentum behind investigating sexual violence at New Zealand universities, and examines institutional risk and anxiety as a key factor in the reluctance to investigate and adequately respond to sexual violence. This chapter then explores the perspectives of key informants and victims/survivors on current responses to sexual violence by universities and university halls, as well as potential avenues for change. The commentaries provided by key informants and victims/survivors are important as they come from first-hand experience with sexual victimisation and provide a solid foundation for informing and developing future responses to sexual violence from universities and university halls. 


\section{Responding to Sexual Violence within University Populations}

In New Zealand, research efforts to identify the prevalence of sexual violence among university students did not come until Nicola Gavey's (1991) survey of first-year psychology students at Auckland University, and it was not until over a decade later that figures on the issue began to surface again (Langley et al., 2003; Cashell-Smith et al., 2007; Connor et al., 2010; Connor et al., Kypri, 2013). This delay is significant, as prevalence studies pave the way for identifying how response and prevention efforts should be designed and implemented. Internationally, there is a plethora of research about current responses to sexual violence among university students (Karjane et al., 2005; Lichty et al., 2008; Quinlan Clarke \& Horsley, 2010). The momentum behind calls for US universities to finally take a stand against sexual violence on their campuses is building (Blain, 2014; Chappell, 2014; White House Task Force to Protect Students from Sexual Assault, 2014); however such momentum has not yet translated to the New Zealand context. The lack of momentum regarding sexual violence among university students in New Zealand is exemplified by a number of recent cases, which will now be discussed.

In June 2012, a female student was raped by two male students in a University of Canterbury hall of residence during a semester-end celebration (Clarkson, 2014). The case made local papers in October 2013 (Clarkson, 2013) and again when both men were jailed for their part in the rape. The case was followed up by an article on February $20^{\text {th }}$ which focused on the risk of rape that young university women face in halls (Dally \& Clarkson, 2014). Neither article appeared in any national newspapers, despite comments from Dr Kim McGregor ${ }^{21}$ indicating that disclosures of similar attacks are regularly heard by her organisation.

In April 2014, two sex attacks occurred in Kelburn, near Victoria University, in the space of 24 hours. The attacks occurred near the Boyd-Wilson field - a field

\footnotetext{
${ }^{21}$ Dr Kim McGregor is Executive Director of Rape Prevention Education in Auckland. Rape Prevention Education works in Auckland as well as nationally to prevent sexual violence through education delivery and health promotion. Further information about Rape Prevention Education can be found at http://rpe.co.nz/
} 
that students have over the years come to name 'Rape Alley'. Salient ${ }^{22}$ has been reporting concerns about the path since 2010 (Mitchell, 2014). The attacks sparked fear of a sexual predator among students and the University extended their safety van for hall residents and Kelburn residents to use for travel between the city and the hall over the Easter break (Hunt, 2014). The attacks encouraged calls for better security near 'Rape Alley' (Hunt, 2014), as well as calls for a coordinated response to sexual assaults and were the impetus for a 300-strong student march in May called 'Let Me Go Home' (Mitchell, 2014). In comparison to recent public uproar around the Roast Busters and nonconsensual sex among teenage girls, there has been little ongoing attention to the issue of non-consensual sex and sexual attacks among university students. In fact, it seems that the public discussion of the stranger sex attacks at Kelburn has ended after the initial hype, with the issue now notably absent, despite the Police still being unable to identify and apprehend the offender.

As well as the stranger attacks at Victoria, there was also a sex attack of an 18 year old woman in August near Massey University's Wellington campus. The incident resulted in Massey University emailing students to remind them about staying safe on campus (Shadwell, 2014a). The 11 safety "tips" delivered to students were controversial, encouraging students to carry whistles and torches, and to wear comfortable running shoes (Shadwell, 2014b). The information was heavily criticised by students at Massey as victim-blaming; students felt that such information placed the onus on victims to protect their own safety, rather than providing advice about consent and telling perpetrators not to commit sexual violence. There has also been little media attention regarding this incident.

The three cases previously discussed are similar in that none of them received dedicated media attention, nor have they publicly put sexual violence on the university agenda. Whilst it is possible that movements may be occurring within universities to actively address the issue of sexual violence within their student populations, there has been little visible change in the discussions occurring in the public arena. This could be viewed as a reactive rather than proactive effort

22 Salient is Victoria University's student magazine, which is funded by students through the student services levy. Salient is available both in print on campus, and online from http://salient.org.nz/ 
for change. This chapter will now explore institutional risk and anxiety as an explanation for the lack of information about sexual violence on New Zealand university campuses, specifically in halls of residence.

\section{Institutional Risk and Anxiety}

Universities have long been seen as proverbial pillars of society, providing safe havens for students and assisting them in the often difficult transition from adolescence to adulthood (Fisher et al., 2007; Fisher et al., 2009). In many ways, universities retain the image of an "ivory tower" in the minds of the public, not just regarding the stereotype of academics within them (Glasby, 2011), but in the very nature of the environment it provides for students. When serious crimes do occur within their boundaries, the ivory tower stereotype shapes the explanation of why such a crime occurred, typically attributing it to disturbed and chaotic individuals rather than as a part of the university context and climate (Fisher et al., 2009). In this sense, public knowledge of crime on university campuses may threaten the belief that crime on university campuses is "the exception to the rule" (Fisher et al., 2009: 1).

Cantalupo (2014: 227) argues that most universities in the United States are "likely aware of the general or national sexual violence incidence rates", however "few have prevalence or incidence data reflecting the size of the problem on their specific campuses." Cantalupo (2014) also argues that the lack of knowledge about sexual violence at university is not due to a lack of surveying; rather, many universities rely on victim reporting, so the lack of reporting is perceived as a lack of incidents. This appears to be the case in New Zealand, where statistics on sexual violence at New Zealand universities are limited. Three key informants commented on this lack of information, with Key Informant 2 noting that due to the rarity of victim reporting, "you never get to join up the dots or get a feeling of the size of the problem." This has implications for not only investigation of the extent of the issue, but the development of robust responses. 
In the US, universities are mandated to publish campus crime statistics by federal legislation, commonly known as the Clery Act. ${ }^{23}$ The idea of the Clery Act is that pressure would be applied to universities that are perceived as dangerous, causing them to ramp up their prevention and security policies to protect students. However, active attempts to identify an issue, or to enhance reporting and prevent sexual victimisation may in fact make a campus look "like it has a serious crime problem" (Cantalupo, 2014: 228). Mikhailovich and Colbran (1999) argue that the identification of sexual violence as a serious issue on a university campus may contribute to an institution's reluctance to investigate or identify the issue at all, so as to appear "less dangerous" than other institutions (Fisher et al., 2009; Cantalupo, 2014).

Given the stereotype of universities as ivory towers and safe havens, there is likely to be anxiety and concern about the impact that negative publicity from sexually violent incidents may have on the overall reputation of the university (Mikhailovich \& Colbran, 1999). This can manifest in institutional reluctance to investigate an issue, or even institutional disregard for published research about university campuses (Wright, 1993). Anecdotally, I have been made aware of the anxieties surrounding researching sexual violence at New Zealand universities in discussions at my own institution and at others. In fact, prior to starting an interview with a key informant, they informed me that they were aware of concerns about my research in terms of the impact that it could have on Victoria's reputation, portraying it in a negative light and dissuading both local and international students from studying here. More recently, I visited another New Zealand university and during discussions with staff, I sensed unease about the topic I was studying, with one staff member commenting that findings from research in this area could put them out of a job and would put parents off sending their students to that university.

Concerns about sexual violence statistics being published may be valid, as negative publicity arising from an incident of sexual violence could have significant impacts for a university, particularly regarding enrolments (Fisher et al., 2009), which would then impact on funding. On the other hand, such

\footnotetext{
${ }^{23}$ The Clery Act is federal legislation that "requires schools annually disclose information about crime, including specific sexual crime categories, in and around campus" (Karjane, Fisher \& Cullen, 2005).
} 
concerns and anxieties could be seen to be putting the interests of the university's reputation ahead of student welfare and wellbeing, thus distorting the reality of safety on their campus. As previous chapters have discussed, the impacts of sexual violence on students living in halls were profound, and such experiences should tip the scales in favour of putting students' welfare and needs first, with the consequences for institutional reputation coming second. What is needed is a New Zealand university to step up and be a pioneer in the area of addressing difficult issues occurring nationwide within all tertiary institutions and associated residences. Framing it as a national issue may help reduce the impact on institutional reputation, and in fact highlight that university as a leader in their field for protecting students' welfare. This pioneer status could actually encourage enrolments, as parents and students may find comfort in knowing that their students are safe, and sets the scene for other institutions to adopt similar changes. This then may open the doors for openness and transparency in responses, as well as developed prevention initiatives on university campuses and in university residences.

\section{Current Responses and Avenues for Change}

Much of the international literature discusses areas of weakness in responses to sexual violence on university campuses (Karjane et al., 2005; Lichty et al., 2008). The interviews in this research asked both key informants and victims/survivors about how they feel New Zealand universities are currently responding to sexual violence, and what potential avenues they see for change in terms of enhancing residents' safety. Both key informants and victims/survivors identified a number of avenues for potential change which could be beneficial for university administrators, and staff involved in running halls of residences. These perspectives will now be explored.

\section{Perspectives of Key Informants}

International literature, predominantly from the US, indicates that responses to disclosures of sexual violence are often managed unevenly (Karjane et al., 2005). The interviews for this research gave an insight into how sexual violence is currently responded to in university halls at Victoria. Four key informants commented on the difficulties involved in responding to sexual violence in university halls, due to the lack of knowledge about the scope of the issue: 
It's a little bit difficult to know what's the best thing to do other than the bits that we do around [Residential Assistant] training when you don't know the nature of the problem. We're not really sure what sort of response is appropriate (Key Informant 3)

I think we respond as able ... we respond as much as we can but only when it is bought to our attention (Key Informant 4)

A lack of information about the pervasiveness of sexual violence on university campuses makes it difficult for university administrators to be involved in developing responses (Cantalupo, 2014), so research in this area is needed to ameliorate this difficulty.

Managing incidents of sexual violence in halls is complex, as not only are there separation needs that must be considered - that is ensuring that victims and offenders do not have to encounter each other in the residence - but there are also requirements in terms of ensuring that victims are aligned with appropriate support services. Further, there are also safety implications involved in instances of sexual violence, not just for a victim but also for other students within the residence. In this sense, there are numerous sets of needs that need to be accounted for when managing sexual violence, which is further complicated by the status of 'alleged' victims and 'alleged' offenders:

And you're thinking about not only the effects on the victim but in terms of a Police kind of mind-set, it's an alleged offence let's say, and it becomes enormously complex if the alleged offender is in the hall. So then you've got two sets of needs that you need to manage, which could be very difficult depending on your worldview of the incident and your own morals and values and things are (Key Informant 6)

Further, three key informants also identified how these difficulties impede the delivery of a smooth and standardised response to sexual violence within university halls:

I think the mechanism for how you get that consistency is probably still reliant on individual managers and individual halls kind of making their own call... because they're so complex (Key Informant 4)

One key informant furthered this, identifying that there is a "lack of consistency in the halls... we now know about probably four, five, or six halls, how each of them have dealt with it is different" (Key Informant 1). Whilst responding to sexual violence is indeed a complex process with a complex set of needs that need to be managed appropriately, this does not mean that a standardised 
procedure for response should not exist, or at the very least be strived for. Key Informant 2 summarises this need well, arguing that there needs to be

...policy in place in ways that that can be properly dealt with. And if there is no way that they can kick them out because of legal reasons or whatever until there's criminal conviction, there needs to be really good management of what they can do to make sure that those people are not having to constantly interact and that they're sensitive to whatever the survivor needs.

Of note, two key informants commented on the efforts made by halls to provide a standardised response. Key Informant 6 commented that:

I wouldn't know the honest answer as to whether it's done perfectly every time, or it has done in the history of Victoria. But I would certainly say in the time that I've been here we've strived to do that as best we can.

Key Informant 6 elaborated on the way that Victoria has strived to do this, and identified the current incident recording processes in halls of residence.

Well we have very clear incident reporting processes ourselves and we use software which has a feature called Clery reports which was based on that principle to document all your incidents and all incidents in all of our halls are documented. Not all are using this software but all of them bar a couple of halls document all kinds of incidents... trying to be more accountable in making sure that if someone's auditing what we're doing they can see what is we're managing and how we managed it... there's a very strong focus on recording what it is that you do.

Having a strong focus on recording and clear reporting processes provides an indication of the extent of sexual violence in university halls, and it is good to see that this is currently in place. However, such reporting software relies on individuals to update the system, and whether this is always adhered to is difficult to comment on and relies on trusted relationships between halls and the wider university. Further research into the scope of sexual violence in halls would provide clearer evidence in support of the development of robust response processes. Ensuring that such processes are adopted by all halls would be beneficial as it would also indicate a move to a standardised response process. Such information would then able to inform future process and policy amendments. 


\section{Education and Awareness-Raising}

As previously discussed in this thesis, young women at university are at an elevated risk of experiencing sexual victimisation (Koss et al., 1987; Fisher et al., 2009; Walsh et al., 2010). Further, some research suggests that young women living in halls of residences may not be aware of the risk that they face, as they deem such residences to be 'safe' and trust that their university halls will take care of them (O’Neill, 2012). This prompted Key Informant 6 to comment about the need for a "high level of awareness" in halls of residence, as well as clear expectations about student behaviour:

There has to be clear expectations in hall environments about the way people should interact with one another and I think that's something that heads of hall and their teams do well. But there's always scope to convey those messages in new ways or to new audiences (Key Informant 6).

As well as awareness-raising, most key informants commented on the importance of delivering education to both students and management about sexual violence. Key Informant 1 felt that there was a strong need for education with students about “around what's okay and what's not” regarding sexual behaviour in halls and previous attempts have been made to deliver prevention education at Victoria. Key Informant 4 commented on the pilot of Sex \& Ethics ${ }^{24}$ that was delivered at Victoria, and the difficulties involved in delivering prevention education on that kind of scale, in a meaningful way for students:

We ran several kind of programmes over several years with most of the halls students and then one with a mixed university group, at least one, might have even been a Uni-Q25 Group actually. And really effective for those who came, but it doesn't have great face attractiveness, you know? And to get students to turn up to lectures, let alone turn up to 4 sessions, 6 sessions in their night time, it ain't gonna happen.

As well as Sex \& Ethics programmes, Key Informant 6 identified that some great work had been done with the Who Are You?26 project in university halls, which requires a lot less effort than delivering Sex \& Ethics. Key Informant 5 felt that

\footnotetext{
24 Sex and Ethics is an Australian-designed education program which focuses on teaching young people about skills in ethical intimacy (Carmody, 2009).

${ }_{25}$ Uni-Q is Victoria University of Wellington's representative group for LGBTQI students on campus.

${ }^{26}$ Who Are You? is a collaborative multi-media campaign and is targeted at university students. The aim of the project is to engage and educate young people about ethical bystanding in instances of sexual violence. More information about the project can be found here http://www.whoareyou.co.nz/
} 
the delivery of information to all first-year students, not just those in halls, would be a positive avenue for change:

Why can't every single first year student see Who Are You? And have an opportunity to discuss it?... if students aren't all seeing the film, then what other ways are making ethical bystander part of what's possible, so not just posters, but that kind of thing. There must be other ways.

Delivering prevention education to all first-year students is important, but how it is delivered is also an important consideration. In some instances, such as self-defence training, the presence of men can exacerbate existing power imbalances between the sexes, and contribute to a loss of focus of the overall aims to empower women (Gidycz \& Dardis, 2014). Therefore, the merits of delivering prevention in single-sex groups should be explored. As well as this, the importance of educating men about sexual violence should not be ignored, as education is crucial to help change men's perspectives about sexual violence and to avoid a victim-centred focus that assumes the prevention of rape is the stopping of women taking part in 'risky' behaviours and 'getting themselves raped'.

As well as identifying new ways to convey messages to students, part of making management more risk aware could come from the delivery of education and training to management. Key Informant 2, in her discussion of training Residential Assistants, felt that sexual violence training was seen as good but not "essential" and acknowledged the difficulties involved with funding ongoing training and support for management. Key Informant 5 felt that delivering education about how to respond to sexual violence was a critical issue:

I think it is how organisations in terms of management etcetera are responding and how they're doing, who they're telling and maybe they haven't thought through clearly about who they should be telling or why... sometimes we do dealing with disclosures workshops for example with different organisations. And I think that something of that nature aye, so if someone comes to us with this, what does this person get from us? But also then who has to know, who really has to know?

The perspectives of key informants here acknowledge the importance of delivering education to students about sexual violence, as well as education for management to ensure that hall staff are well-equipped to deal with sexual violence disclosures. Such education should be viewed as necessary and 
essential rather than just an optional extra given the devastating impacts that sexual violence has on victims.

\section{Coordinated Response to Sexual Violence}

Universities have been described as best placed to respond to sexual violence due to the expertise available on university campuses, as well as the centralised locations of support services for students (Mikhailovich \& Colbran, 1999; Sabina \& Ho, 2014). Harking back to previous calls by the student body for a more coordinated response to sexual assault following the Kelburn sex attacks described above, Key Informant 4 commented on the merits of having a more consistent 'go to' group for halls of residence that are managing a disclosure of sexual violence:

I think there would be some merit in having more of a consistent go to group for halls, hall managers to consult with around a response process and protocol. I think that probably happens to some extent at the moment you know, with people like [senior management].

Having a more consistent go-to group for consultation in the event of a disclosure also requires a robust policy to direct how disclosures will be managed and how the needs of both victims and offenders will be balanced, so strengthening this policy is a crucial starting point. In the United States, there has been a substantial amount of literature investigating the role of specialist Sexual Assault Response Teams (SART). SARTs are a coordinated mechanism aimed at improving the ways victims of sexual violence are treated by the criminal justice system (Clevenger, 2014). The SART works to ensure that all victims are linked up with the appropriate support services that they need at the point of disclosure (LaMonica \& Pagliaro, 2013). Although SARTs are generally run in the community, and thus experience collaborative difficulties (Greeson \& Campbell, 2013), given the availability of staff expertise regarding sexual violence at university, as well as the location of on-site support services, perhaps university halls and university staff could be encouraged to look at the development of a SART response in the university context. Such a response team could help ensure both a seamless response, victim linkage to support services as well as a limited number of people that would be informed about it, which could help support the privacy needs of both victims and offenders. 
Criminal justice system responses to victims of sexual violence are gruelling and unnecessarily harsh (Jordan, 2008; Temkin \& Krahe, 2008; Jordan, 2013). The harsh treatment of victims by the adversarial criminal justice system has encouraged thinking about alternative processes for dealing with sexual victimisation, and there is a building body of research that explores the role that restorative justice processes could play in dealing with sexual violence cases (Daly \& Curtis-Fawley, 2006; Julich et al., 2011). However, there is also concern around the capacity of restorative justice processes to respond to sexual violence cases (Tinsley \& McDonald, 2011). New Zealand currently has restorative justice processes for cases of sexual violence through Project Restore (Julich et al., 2011), and Key Informant 4 felt that there could be some merit in considering restorative justice processes for managing sexual violence in halls of residence:

We've got this shit system to deal with sexual assault. And it's the best we've got, and I think that the university is understandably really cautious about offering anything as an alternative. I personally think we should get over ourselves you know, and actually...be more courageous... if students choose not to go down a Police track, and who would you know, then it'd be cool for us to have a robust, brave, solid way of meeting with them and to get some restorative justice out of it if you like. Tricky though.

Recent work by Koss, Wilgus and Williamsen (2014) explored the use of restorative justice processes in campus sexual misconduct in the United States. The authors note that

although RJ programs are present on many campuses as evidence by the level of participation in evaluation research, few or none of them have formally integrated sexual misconduct. Many institutions that are using or open to trying RJ options for some forms of misconduct have excluded sexual assault because they view it as so unique that it involves special handling (Koss et al., 2014: 254).

Concerns about universities dealing with sexual violence cases in-house could arise if universities were to adopt a restorative justice process for responding to sexual violence, and this was certainly a concern identified by Key Informant 4 . However, given the current ways in which sexual violence is managed by university halls, there could be benefits in an alternative model for sexual violence victims/survivors in these contexts. There would need to be a 
willingness by a university to be courageous and take a step forward in alternative processes, as well as significant research conducted to assess the appropriateness of such a process, but it does open up potential opportunities should interest in this area arise in the future.

Key informants have identified the lack of information about sexual violence among university students, as well as the current responses to sexual violence among students. Further, they have highlighted where responses have not worked well, and have suggested that there is merit in exploring education and awareness-raising efforts for both students and management, the development of coordinated responses to sexual violence at the university, and the potential benefit in restorative justice processes for halls of residence to manage sexual violence. These recommendations share similarities and differences to those put forward from victims/survivors, which this chapter will now explore.

\section{Perspectives of Victims/Survivors}

There is a lack of New Zealand information about the ways victims/survivors want their university halls to respond to them in the event of a disclosure, or enhance their feelings of safety within the residence. This literature gap encouraged the inclusion of victims'/survivors' perspectives on the ways that university halls could make living environments safer for residents. These views will now be explored in turn.

\section{Validation and Agency}

For many victims/survivors, the importance of their experience being acknowledged and validated cannot be overstated (Walsh et al., 2010). Whilst validation and acknowledgement of disclosures would be the ideal response from everyone that receives a disclosure, in practice it is not the case. A substantial amount of research with university students cites a fear of not being believed as a common barrier to disclosure (Fisher et al., 2009; National Union of Students, 2010; Sabina \& Ho, 2014), and recent research suggests that betrayal by institutions to prevent or respond adequately to sexual assault can actually exacerbate feelings of trauma (Smith \& Freyd, 2013). Dana's experience with disclosure - feeling that management were minimising her feelings and 
blocking her at every point - informed her views on the ways that management should respond:

I think the first thing they should do is acknowledge it. Acknowledge that it's a crime and that something has happened to somebody. Don't remove the person from the equation. Don't like take them out of it... And genuinely mean it, genuinely mean the acknowledgement.

Dana's commentary on the importance of acknowledging victims/survivors and including them in the process of investigation is a resounding one, and provides important directives to institutional staff - from the perspective of a victim/survivor - about the ways in which disclosures should be managed. Dana's views mirrored the ways that disclosures were described as being managed in university halls:

From that point, the minute that that disclosure takes place, we have to start thinking about how best to manage that... what's going on here because I'm the person that's been privy to this information? Who will I need to contact? Will I need to get somebody in? ... How am I going to engage with the person that's disclosed in a way that makes them feel like they've got some degree of control over it? Thinking of it from a process level from that point of disclosure (Key Informant 6)

Importantly, Key Informant 6 describes the importance of ensuring that victims are given some sense of agency over their disclosure and control over the process, precisely what Dana felt was needed for her. However, as previously noted, key informants were not confident that a seamless response process had been adopted every time a disclosure was made, and this is certainly the case for Dana. Whilst Dana's experience with disclosure may not be generalisable to the experiences of others who have disclosed, her experience should not be ignored and should be used to ensure that such failings do not occur in the future.

\section{Confidence and Trust in Management}

All of the women who took part in this research discussed the importance of having confidence and trust in management at the hall. Dana - the only participant who disclosed to hall management - felt that management was "blocking" her at every point which impacted on her ability to trust management, and she became "suspicious" of their agendas. Indi reflected on the way that hall management handled a situation where a young woman was raped, but the perpetrator was allowed back at the hall for a second year: 
It was kind of kept under wraps but it sort of got out through gossip that it'd happened and everyone was really pissed off that he was allowed back. So yeah for a start, dealing with those situations properly.

Indi's reflection identifies the importance of having trustworthy individuals charged with managerial roles in university halls. Without that trust, this too may contribute to underreporting as previous knowledge of how situations may become the expectation, which is problematic when previous situations have not been handled adequately. Indi's comments reaffirm the importance of getting things right the first time around, otherwise the implications can be farreaching and may become the expected response in the event of a disclosure.

Fay felt that management within the hall needed to be more approachable and "make an active effort to be more good cop than bad cop." Further, Indi commented on the attitudes displayed by management at her hall:

One of my friends is like a black belt in karate and at the start of the year she was like 'oh maybe we should set up a little thing with people from our hall, like with the girls, to do like self-defence'. And then the [Hall Manager] said no to it because she said 'if girls learn self-defence, then they're more likely to put themselves in dangerous situations'.

Attitudes such as playing bad cop, or blaming victims for putting themselves in danger works to silence victims by discrediting their experiences (Schwartz \& DeKeseredy, 1997). Further, the comments provided by Indi's hall manager are not synonymous with academic literature now emerging in the United States, which finds that self-defence training for female students can actually be empowering rather than enhancing feelings of fear or encouraging participation in risky behaviour (Cermele, 2004; Gidycz \& Dardis, 2014). This is problematic in halls of residence as the absence of reporting can be construed as the absence of an issue (Ottens, 2001; Argiero et al., 2010), which reduces opportunities for change. To combat such attitudes, Bianca commented that ongoing training of management would ensure that management did not "forget" things. Such education would be beneficial to ensure that students feel comfortable and trusting of hall management.

\section{Education and Information}

Delivering education about consent and ethical bystanding has been a focus of sexual violence prevention initiatives, particularly with university students 
(Vladutiu et al., 2011; Brown, Banyard \& Moynihan, 2014; Moynihan et al., 2014). The bulk of literature regarding sexual violence prevention is in the US, and there is a lack of information regarding prevention and education delivery in New Zealand university halls. However, three of the women interviewed for this research commented on the importance of equipping students with information about sex and sexual violence:

Basically just drive home the message that sexual comments are not acceptable or appropriate... I don't know how to teach not to slut-shame but yknow maybe try that in some way... I just think that just let people know what's okay and what's not okay... sexual comments are not okay (Fay)

Indi identified a number of areas that could have benefited from education and information, and identified the importance of such information being delivered early, and in human terms:

I think with sexual violence it should just be educated about early. Like this is the proper way to treat people. Just basic human courtesy of don't be a dick. You'd think it would be obvious but apparently it isn't.

Indi also commented on the importance of delivering information about ethical sex to students living in halls, due to complexities and differences in knowledge about consent:

Coz I think also a lot of people don't understand what consent really means. I mean, you have to be able to give consent in the absence of a yes, I mean a no isn't a yes. Little things like that people don't really understand.

Due to the dearth of New Zealand literature regarding the delivery of sexual violence education to New Zealand university students, it is difficult to speculate whether such information is provided. Interviews with key informants for this research indicated that some information had been delivered through the Sex \& Ethics pilot at Victoria and the use of the Who Are You? material. However, a dedicated and concerted effort to encourage students to think about ethical sex and what constitutes sexual violence would be positive to see and is in line with the wishes of students who have directly experienced sexual victimisation in halls of residences. 


\section{Summary}

This chapter has identified institutional risk and anxiety as one of the key factors that contributes to the lack of investigation of sexual violence at New Zealand universities and has examined the complexities involved in responding to sexual violence in university halls of residence. This chapter has also provided a commentary by both victims/survivors and key informants about how well universities are currently responding to sexual violence, and areas of improvement for the future. Whilst there are some measures currently in place to respond to sexual violence, it appears that responses are only provided in a reactive manner. That said, key informants and victims/survivors provided a number of ways that response processes could be improved and these findings are unique as they come from the voices of those with first-hand experience in experiencing and dealing with sexual violence among university students. These findings may be beneficial for universities and university hall management in the development of appropriate response protocols for sexual violence, which have the needs identified from those with expertise at the forefront of discussions. 


\section{Conclusion}

But I just wanted to tell you because I was like [pause] if your Master's thesis or whatever gets in the right hands, then maybe the university could put more support systems for stuff like this within hostels (Fay)

This final chapter brings together the key issues discussed in the previous four chapters and addresses the implications that these findings have in relation to sexual violence in New Zealand university halls. Research on women's experiences of sexual violence at New Zealand universities, or their associated halls, has not previously been conducted. In fact, little research, quantitative or qualitative, exists about sexual violence among New Zealand students. Whilst this research is largely exploratory in nature, this research contributes to this literature void and emerges at a time when momentum is building, especially in the US, to address sexual violence among university students. Perspectives from both the women interviewed and key informants form the basis of this study. Using these as a starting point, this thesis argues that sexual violence in university halls is a significant risk for young women, causes devastating impacts and remains largely unreported to the police and hall management. This thesis also highlights the urgent need for adequate responses and prevention delivery to enhance women's safety in university residences. 


\section{Identifying the Scope of the Issue}

As this thesis has established, there is a paucity of research on the prevalence of sexual violence at New Zealand universities or in their affiliated halls of residence. This lack of information exists, despite international momentum calling for universities to respond to high prevalence rates, and a number of recent high-profile cases of victimisation either in, or on, New Zealand university campuses or halls (Dally \& Clarkson, 2014; Mitchell, 2014; Shadwell, 2014a, 2014b). The lack of knowledge in this area is of concern, as a lack of information could preclude recognition of the extent and prevalence of such violence (Ottens, 2001; Argiero et al., 2010). This then hampers the development of best practice response mechanisms to incidents of sexual violence, as well as ongoing and dedicated prevention efforts with university students.

Key informants in this research called for more information about the levels of sexual violence in universities and university halls, as without this base, and the rarity with which it is officially reported, it becomes difficult to know how best to respond to and manage incidents when they arise. Further, it may cause universities and their halls to be disinterested in the issue, and treat it as a rare and random act, rather than a more common experience for university women that demands urgent attention. In order to ensure the safety of women at university and living in halls of residence, a thorough understanding of the levels of sexual violence, and what contributes to them, is needed. This thesis provides a platform for future research in this area. An investigation on a national scale, similar to the National College Women's Sexual Victimisation Study (Fisher et al., 2009), would be a beneficial starting point for identifying the extent of the issue at New Zealand universities. Further, university-level campus climate surveys (Karjane et al., 2005) would also be useful to identify institution-specific needs.

\section{Living in a Hall of Residence: Risky Living?}

The living environments provided by halls help set the scene for a student's overall university experience. It is therefore crucial that areas of risk are minimised to ensure women's safety. Key informants' perspectives in Chapter 
Four identified particular risk factors within the hall environment itself. Interviewees described the population of students in halls as a highly sexualized group, in an age bracket (generally 17-24) that typically engages in more risktaking than other groups, with alcohol being identified as one of the biggest risk factors for sexual violence. Further, key informants also identified that there is a lack of education about sex and consent among student populations, which is further complicated by an atmosphere of sexual pressure within hall environments. The latter encourages students to partake in sexual activity to fit in and feel accepted, at a time when they are likely to be exploring sexual boundaries.

Key informants identified the 24/7 nature of the hall environment as a contributing risk factor, noting that such an environment means residents see others more regularly, and behaviours within that environment may be different than they would if a student was only attending campus. These changed behaviours also happen in an environment where mixed-gender or coeducational housing predominates, and where students are constantly interacting in both public and private spaces at a time of increased freedoms and a lack of parental safeguards. This makes it difficult for women to claim a safe space within their hall environment, and contributes to difficulty avoiding a perpetrator in such a public environment.

Although there remains a lack of data regarding the specific risk factors in halls of residence, alcohol use among student populations has consistently been identified as a risk factor for sexual violence (Adams-Curtis \& Forbes, 2004; Cashell-Smith et al., 2007; Connor et al., 2010). Further, the lack of awareness about consent and the presence of rape-supportive beliefs has consistently been discussed in literature regarding female student's experiences of victimisation (Ottens, 2001; Burgess, 2007; Argiero et al., 2010). In this vein, risk factors identified by key informants contribute to this existing literature base, and provide an overview of risk factors within New Zealand populations to be considered for harm-reduction measures in university halls.

Chapter Four also documented the ways that these risks became realities for 
women in their hall environments. The women identified a number of situations where they witnessed the blurred lines between alcohol and consent, and they commented on situations where sexual activity had taken place, but there was uncertainty about whether a person was actually capable of giving consent in that situation. The women also described their halls as a sexual environment, and highlighted the existence of both sexist and sexually abusive behaviour. Most of the women interviewed experienced such behaviour for a continued period during their time in the hall, with ongoing, unwanted sexual comments from men in public spaces being a common occurrence. The public nature of these comments made it difficult for women to counter or stop them. Further, the women discussed a lack of sexual privacy within the hall, and the ease by which sexual gossip was spread throughout the residence. Lastly, the women described incidents of sexual violence occurring in the hall, either personally experienced or heard about, which in their views were not handled adequately by management when reported.

In essence, the women described an unsafe environment that perpetuated gender-based violence and uneven power relations between men and women, due to the ease with which men were able to perpetrate this behaviour against them. This inequality severely limited the women's ability to enjoy the hall or their overall university experience (Day, 1994; Kelly \& Torres, 2006). This contradicts the very ideal of university halls as being safe, homes away from home for students to transition to university life and to enjoy their university experience to the fullest. Changes must be made to improve and enhance the safety of women within halls of residences, and the minimisation of harm must be seen as a need, not a want.

\section{The Consequences of Sexual Violence}

Sexual violence can have devastating impacts for victims, and the women interviewed for this research highlighted the tremendous impact that their experience had, as discussed in Chapter Five. What they were subjected to in their halls affected them psychologically, and the women felt fearful, anxious, and some felt a loss of confidence and trust, which for one woman was reinforced from prior victimisation. Further, some reported difficulties sleeping 
and some experienced flashbacks and panic attacks in the aftermath of victimisation. These impacts made women question aspects of their own identities, which is particularly challenging for women to experience at such a transitional time in their lives, and without the existing support that may have existed when living in the family home. The impacts that these women reported are not dissimilar to wider research about the consequences of sexual violence (Kelly, 1988; Culbertson et al., 2001; Sampson, 2002; Yuan et al., 2006; Basile \& Smith, 2011; Calhoun et al., 2012).

As well as affecting them psychologically, their victimisation carried a huge social weight as well, impacting on their interpersonal relationships, especially with their friends and intimate partners. This is particularly concerning as support from friends, family and intimates following victimisation can be vital to a victim's recovery (Campbell et al., 2009). Further, this thesis acknowledges the very real impact that sexual victimisation can have on academic performance at university. Although this remains an area that has not received significant research attention (Ottens, 2001; Jordan et al., 2014), in a country such as New Zealand which has such high numbers of students that do not complete their degrees that they initially enrolled in (Scott, 2009), there would be merit in exploring sexual violence as a possible contributor to students failing to complete their studies.

While some people may view the experiences included in this thesis as on the 'less-serious' end of a spectrum of sexual violence, this does not mean that the consequences of such violence were negligible. For the women who took part in this research, the impacts of sexual victimisation were huge. They questioned their own identities, questioned the loyalties of their friends and intimate partners, and felt unsafe in their residence. The impacts meant that their experience significantly impacted on their ability to experience fulfilment at university, and for some acted as a barrier to successfully completing their studies. The women's accounts of their experience serve as a strong reminder to universities, and their associated halls, of the importance of ensuring that harmminimisation measures are in place to protect the wellbeing of students in their care, as well as appropriate referral pathways to support services. 


\section{Sexual Victimisation Disclosure: Speak Out or Stay Silent?}

Chapter Six discussed women's experiences with disclosure, noting that only one formally disclosed to the Police, however all of the women involved disclosed to less official sources. The lack of reporting to the police is an acknowledged, wide-spread issue, and is also an issue among student victims (Krebs et al., 2007; Fisher et al., 2009; Edwards et al., 2012). Although this research used a very small sample, which therefore impacts its generalizability, one woman provided a stellar review of the way she was treated by Police, which was reiterated by a key informant who commended their working with university students. However, the experiences of women with reporting to the police are not always positive, and New Zealand data suggests that women's experiences of talking to the Police are often negative and can cause further negative impacts (Jordan, 2012).

Participants discussed a number of barriers to reporting sexual victimisation, such as feeling that what happened was not a crime, it could be handled independently, it would not be taken seriously by management, and uncertainty about how to report. These barriers resonate with the existing literature on students' experiences of disclosing sexual violence (Fisher et al., 2009; Zinzow \& Thompson, 2011). The women also identified a number of barriers to reporting to their existing social supports. They felt fearful of negative social reactions, as well as negative repercussions, and were concerned about others finding out about what happened, which inhibited their ability to disclose. These barriers have also been documented in previous research with students (Orchowski \& Gidycz, 2012; Edwards et al., 2014), so this thesis supports these existing findings. The identification of barriers to disclosure is the first step to helping break them down, so this research has utility for halls of residence, and the wider university, for encouraging reporting.

Encouragingly, most women reported that their experience of disclosing to those around them was positive. Most participants reported feeling a sense of legitimacy when they disclosed to those around them, as well as identifying helpful support from intimates, friends and family postvictimisation. However, two women reported negative experiences with disclosure, and uniquely, the 
public nature of the hall environment, combined with the ease that sexual information was shared, meant that for one participant, she did not need to disclose as her experience was already common knowledge. Such publicity caused a negative response from friends, who staged an 'interfriendtion'. This 'trial by friendship' is a salient indicator of the importance of delivering information to students about how to respond to disclosures of sexual violence, and such a finding has implications for universities and university halls in terms of educating students about the issue and the potential avenues for support.

The women interviewed in this research also identified barriers to accessing support services at the university, or in the wider community. Only one woman reported seeking counselling services about what was going on at the time, and her discussions with the counsellor were more about the social impacts she was experiencing, rather than the incidents themselves. Women chose not to use the counselling services or to seek help as they felt that they could handle it independently, had concerns about confidentiality, or they felt that their experience was not serious enough or would not qualify for specialist services. Again, these barriers have been noted in other research about women's helpseeking behaviours postvictimisation (Nasta et al., 2005; Fisher et al., 2009), so this thesis supports this previous work. These findings warrant further investigation and education efforts to help educate women about the services available at university, what they are for, how to access them, and how confidentiality can be assured.

All of the women in this research identified particular men who displayed problematic behaviours toward them, in both public and private spaces. Some of the women also commented on the men's notoriety in the hall for acting inappropriately. In this sense, it is likely that the women interviewed for this research are unlikely to be the sole victims of these behaviours, and others are also likely to be experiencing similar impacts to the women who participated. Therefore, underreporting of sexual violence contributes to continually unsafe hall climates and ongoing perpetration of sexual violence in university populations and wider society. Such a finding warrants further research attention, and also could lead to universities and affiliated residences playing a 
more active role in learning how to break down the barriers to disclosing that the women identified, to enhance the safety of not just victims, but the wider community.

\section{Dealing With Sexual Violence: How to Respond?}

Responding to, and preventing, sexual violence is just as complex as the issue itself. Responding to sexual violence is further complicated when there is a paucity of knowledge or awareness about the scope of the issue. Chapter Seven highlighted these complexities, and discussed the role that institutional anxieties about reputational damage may have in the continued absence of sexual violence data at New Zealand universities (Mikhailovich \& Colbran, 1999; Cantalupo, 2014). Concerns about a domino effect impacting on university enrolments, hall enrolments and the attraction of international students are a valid concern, however the protection of students welfare and wellbeing is of paramount importance, and such a focus should come before institutional reputation. Approached from a different perspective, it is likely that open acknowledgement of sexual violence risks and clear evidence of preventive strategies and responses would instead enhance a university's reputation as an arena committed to students' wellbeing.

Enhancing responses to sexual violence has been started at Victoria, and key informants identified that the implementation of Residential Assistant training has been a successful initiative. However, key informants also noted that they are currently only responding as able, only when incidents are brought to their attention, and there is not a standardised response process in place currently. Further, key informants also acknowledged the complexity involved in responding to sexual violence, as there are separation needs, support needs and safety implications for residents in the hall and the wider university community. In this vein, it is imperative that sexual violence responses are robust and capable of managing such a complex issue in a way that supports victims fully, but also acknowledges that there may be needs for an offender and also the wider community. 


\section{Implications of this Research}

This research, although small in sample size, has provided a rich qualitative data set which provides the first insights into university women's experiences of sexual violence in New Zealand university halls. As previously discussed, the women interviewed for this research reported distressing accounts of repeated and unwanted sexual attention and harassment, experiences of sexual assault, and a lack of sexual privacy regarding their sexual histories. These experiences had profound impacts, and the majority went unreported to the police or other 'official' channels such as hall management. As well as this, most women did not receive specialist help following their experiences. Both key informants and the women interviewed identified how some current responses to sexual violence were working well, however they also made a number of recommendations about how to better protect students living in halls, as detailed in Chapter Seven.

Although there is little known about the delivery of sexual violence prevention initiatives at New Zealand universities, this research highlights the ways prevention does not appear to feature strongly on the university's agenda. Prevention efforts have occurred sporadically and their implementation has been the responsibility of key staff within the university pushing for it, rather than a dedicated and well-resourced drive by the university. These findings echo recent research at Auckland University (Smith, 2013) which indicates the national significance of the issue, and does not localise the issue to Victoria. However, the findings from this research may have strong implications for New Zealand universities regarding the ways that universities respond to sexual violence in the event of an incident, as well as the investigation and development of meaningful sexual violence prevention initiatives within their halls and the wider university population.

While preventing sexual violence is as complex as the issue itself, this does not mean that efforts should be half-hearted, or ignored, because of their difficulty. In fact, universities should be aware of the multitude of preventive efforts that can, and should, be delivered. Brief, one-session interventions designed to educate students about changing their attitudes toward sexual violence have been shown not to work with university students (DeGue, 2014). Whilst no 
single prevention effort is the whole answer to tackling sexual violence, their benefit when used as part of a concerted and dedicated initiative must be noted.

I recommend the investigation and development of robust and standardised response protocols, as well as four key areas for prevention efforts, using halls of residence as a prevention site due to the locality of students and the ease by which they can take part. Addressing halls of residence as pilot sites will also help draw out the needs for the wider university population. These recommendations will now be listed in turn, and should be considered not only by Victoria, but also other New Zealand universities and affiliated residences.

\section{Recommendations for Best Practice in Responding to Sexual Violence}

Following a thorough review of the literature regarding responding to, and preventing, sexual violence among university student populations, as well as interviews with victims/survivors and key informants, I have identified a number of recommendations that I believe would go some way to ensuring that allegations of sexual violence are responded to in the most appropriate way. These recommendations are by no means presented as the only option, however they are important to consider given they are shaped by the views of victims/survivors and key informants who hold first-hand experience or knowledge in either experiencing victimisation or responding to it.

\section{Recommendation 1: Comprehensive Sexual Misconduct Policy}

University sexual assault policies should be easily understandable, accessible and widely distributed to students. They should define sexual violence clearly, rather than labelling it under sexual harassment, and should detail expectations of students' sexual conduct (Karjane et al., 2005). Further, they should outline how to report sexual violence, list the available support services both on campus and in the wider community, emphasise confidentiality and detail the consequences for breaching the misconduct policy. The benefits rigorous sexual assault policies should be acknowledged by all universities, and encouragement to ensure that universities nationally are involved in this dialogue could be a role for the New Zealand University Students' Association to play to encourage a 
pan-university approach.

Recommendation 2: Development of a Standardised Response Protocol for Sexual Violence Incidents

Standardised responses to sexual violence should be developed, and university halls should be mandated to adhere to these guidelines. Responses to sexual violence should be managed by a skilled team of responders, perhaps in a similar set-up to the SART discussed in Chapter Seven, which would ensure victims' voices are validated, help limit the number of people involved in the process and help protect both the victim's and the alleged offender's confidentiality. Responders should be well-informed about referral pathways to sexual abuse support agencies both at the university and in the wider community, should students wish to use them. Further, victims must be guaranteed control over both the pace of the process, as well as the decisions made throughout about reporting under no coercion. Having a written protocol that ensures this enhances the likelihood that victims will have a "coordinated, consistent, victim-centred response" (Karjane et al., 2005: 13).

\section{Recommendation 3: Auditing of Responses to Sexually Violent Incidents}

All responses to sexual violence should be appropriately recorded, and audited by an appropriately trained manager independently to ensure that a smooth and seamless response is provided to students in the event of a sexually violent incident. Audit recommendations should be provided to all staff involved in protecting students' welfare so that learning is shared.

Recommendation 4: Continued Training of Management and Residential Assistants

Training of management and Residential Assistants should continue to ensure that staff who may be responders to a sexually violent incident are equipped with the right knowledge and capability to respond to such an event. Training should also be supplemented throughout the university year with refreshers, especially after an incident of sexual violence has occurred. 
Recommendation 5: Ongoing Relationships with Community Agencies and the Police

Community agencies such as Rape Crisis and Sexual Abuse HELP Foundation provide expert advice and support for victims/survivors of sexual violence. The Police also provide a wealth of information about official reporting protocols and the criminal justice system response to official complaints. Relationships with community agencies and the Police need to be as strong as possible to ensure that students are able to be referred to such support, if they wish to do so. Further, such relationships mean that expert advice can be given to ensure that a victim-centred approach is taken at all times when responding to sexual violence.

\section{Recommendation 6: Sexual Violence Disclosure Education}

The majority of students disclose sexual victimisation to their existing social supports, most often peers (Orchowski \& Gidycz, 2012). Therefore, it is important that all students receive education about how to respond to a disclosure, so they are able to provide the best support and guidance for victims, to avoid responses such as 'interfriendtions'.

\section{Recommendations for Best Practice in Prevention of Sexual Violence Recommendation 1: Consent Workshops and Sexual Education}

Education about consent, respectful sexual behaviour and risk factors for sexual violence should be delivered to all university students. Gearing prevention workshops to single-sex groups is an important consideration to ensure that students feel comfortable discussing issues and there is a limited imbalance in power between the sexes. Further, single-sex delivery also ensures that responsibility is put on men to become engaged in helping prevent crimes which are overwhelmingly perpetrated by men (Karjane et al., 2005).

Workshops should be made compulsory for first-year students, perhaps as part of the Orientation Week activities, and refreshers should also be offered. Workshops should be delivered by trained facilitators, and using students as well as staff to deliver such information could be beneficial in establishing rapport. Previous efforts by institutions in the United States have used theatre 
to deliver prevention efforts, and such use could be helpful in keeping students engaged.

\section{Recommendation 2: Ethical Bystander Education}

The importance of delivering education to students about being an ethical bystander, such as in the Who Are You? campaign, highlights how students can play an active role in protecting their friends and those around them. Teaching students about how to intervene safely increases awareness and keeps students aware of the risk of sexual violence.

\section{Recommendation 3: Self-Defence Training}

Self-defence training for women has been shown to actually empower them by teaching them how to resist incidents of sexual violence when they occur (Cermele, 2004; Gidycz \& Dardis, 2014). Whilst some have criticised selfdefence training as placing the onus on the victim to protect themselves, it has utility when delivered within the context of a feminist empowerment model and among a number of other preventive efforts. A self-defence training course could be offered in halls of residences and evaluated for further delivery in the wider university community.

\section{Recommendation 4: Ongoing Research}

Given the limited amount of information about prevention efforts, ongoing research attention should be given to developing, implementing and evaluating prevention programmes (DeGue, 2014) at New Zealand universities. This ensures that resources are being best utilised, as well as remaining flexible to adapt to changes in university populations. Further, lessons learned from prevention programmes should be shared with other universities to have a positive impact on students' lives around the country (DeGue, 2014).

\section{Looking to the Future}

As discussed throughout this thesis, the international literature on students' experiences of sexual victimisation is significant, especially in the United States (Koss et al., 1987; Kilpatrick et al., 2007; Krebs et al., 2009; Fisher et al., 2009; Walsh et al., 2010), however the literature base is sparse in New Zealand. This 
thesis contributes to the small New Zealand literature base (Gavey, 1991; Langley et al., 2003; Cashell-Smith et al., 2007; Kypri et al., 2007; Kypri et al., 2009; Connor et al., 2010; Connor et al., 2013; Smith, 2013) by providing the first qualitative inquiry into female students' experiences of sexual violence in halls of residence. The very personal experiences shared by women interviewed for this research, in conjunction with the perspectives provided by key informants, provide a solid foundation for the investigation of sexual violence at New Zealand universities and in affiliated halls of residence. Further, the devastating impacts that the women detailed in this research signal the need for ongoing prevention efforts to protect the wellbeing and safety of not just victims of sexual violence, but also all students living under the care and protection of the university. By developing robust response protocols to incidents of sexual violence and implementing well-resourced and researched prevention initiatives, universities signal to both current and prospective students, and their parents, that they are leaders in their field and dedicated to ensuring the safety of their students.

This thesis emerges at a time when sexual violence has become an issue of national discussion, and recent moves by the Minister of Justice, Amy Adams, have indicated that alternative justice system responses to sexual violence will be investigated again as a top priority by the New Zealand Law Commission (Cowlishaw, 2014). It is hoped that the publication of this thesis contributes to a discussion of sexual victimisation among university students, especially those living in halls, and gains the momentum needed to encourage change within New Zealand tertiary institutions. 


\section{Appendices}


Appendix A: Ethical Approval

TE WHAKE WANANGA O TE OPOKO O TE IKA A MAUI

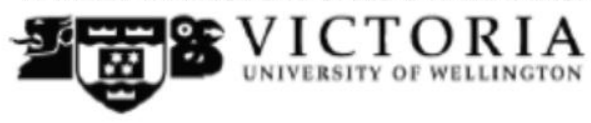

MEMORANDUM

Phone $\quad 0-4-4635676$

Fax $\quad 0-4-4636130$

Email kathynelson 6 vuw.acnz

\begin{tabular}{l|l}
\hline TO & Samantha Keene \\
\hline COPY TO & Jan Jordan \\
\hline FROM & Dr Kathy Nelson, Deputy Convener, Human Ethics Committee \\
\hline
\end{tabular}

\begin{tabular}{|c|c|}
\hline DATE & 20 September 2013 \\
\hline PAGES & 1 \\
\hline SUBJECT & $\begin{array}{l}\text { Ethics Approval: } 20176 \\
\text { Female students' experiences of sexual violence within university } \\
\text { halls of residence }\end{array}$ \\
\hline
\end{tabular}

Thank you for your application for ethical approval, which has now been considered by the Standing Committee of the Human Ethics Committee.

Your application has been approved from the above date and this approval continues until 10 August 2014. If your data collection is not completed by this date you should apply to the Human Ethics Committee for an extension to this approval.

Best wishes with the research.

Kathy Nelson

Human Ethics Committee 
MEMORANDUM

\begin{tabular}{l|l}
\hline TO & Samantha Keene \\
\hline COPY TO & Jan Jordan \\
\hline FROM & Dr Allison Kirkman, Convener, Human Ethics Committee \\
\hline DATE & 11 November 2013 \\
\hline PAGES & 1 \\
\hline SUBJECT & $\begin{array}{l}\text { Ethics Approval: 20176 } \\
\text { Female students' experiences of sexual violence within university } \\
\text { halls of residence }\end{array}$ \\
\hline
\end{tabular}

Thank you for your request to amend your ethics approval. This has now been considered and the request granted.

Your application has approval until 10 August 2014. If your data collection is not completed by this date you should apply to the Human Ethics Committee for an extension to this approval.

Best wishes with your research.

Allison Kirkman

Human Ethics Committee

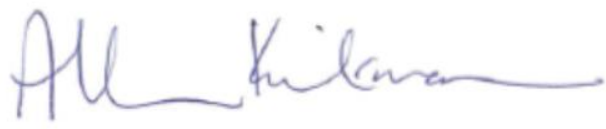

Phone 0-4-4635676

Fax $\quad 0-4-4635209$

Email Allison.kirkmanthruw ac.ne 
MEMORANDUM

Phone $\quad 0-4-4035070$

Fax 04-4035200

Emal Allison kirkmuntwuwacne

\begin{tabular}{l|l}
\hline TO & Samantha Keene \\
\hline COPY TO & Jan Jordan \\
\hline FROM & Dr Allison Kirkman, Convener, Human Ethics Committee \\
\hline
\end{tabular}

\begin{tabular}{l|l}
\hline DATE & 27 March 2014 \\
\hline PAGES & 1 \\
\hline
\end{tabular}

\begin{tabular}{l|l}
\hline SUBJECT & $\begin{array}{l}\text { Ethics Approval: 20176 } \\
\text { Female students' experiences of sexual violence within university } \\
\text { halls of residence }\end{array}$ \\
\hline
\end{tabular}

Thank you for your request to amend your ethics approval. This has now been considered and the request granted.

Your application has approval until 20 August 2014. If your data collection is not completed by this date you should apply to the Human Ethics Committee for an extension to this approval.

Best wishes with your research.

Allison Kirkman

Human Ethics Committee

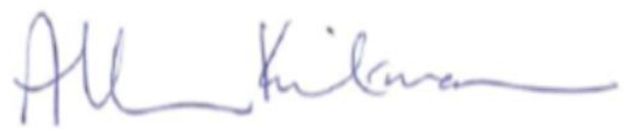




\section{Appendix B: Recruitment flyer}

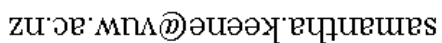

\&9t (†o) do \&98t $98^{\circ}$ ( $\left.\angle z \circ\right)$ әиәәу вцдише

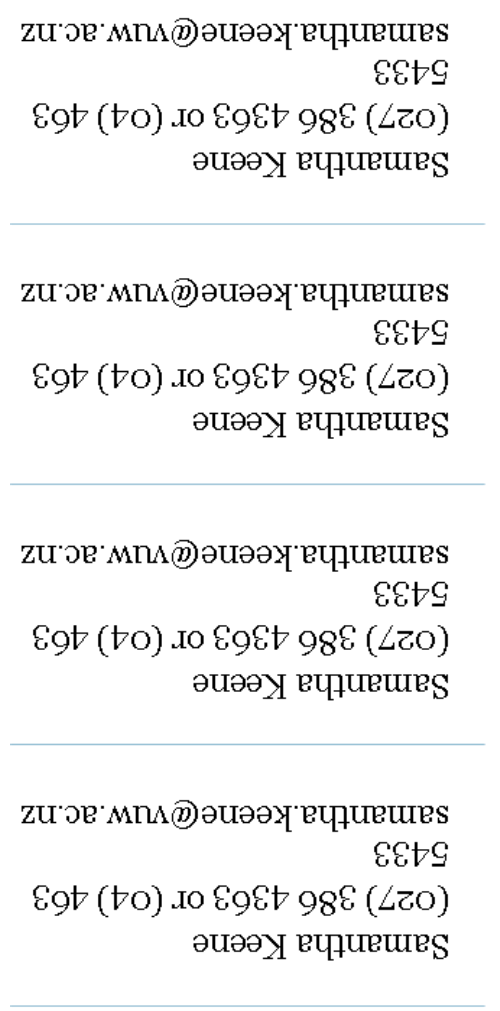

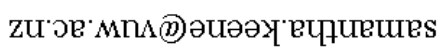
$8 \& t 9$

Eqt (to) do E98t 988 ( $\angle z \circ)$ әиәәу вцдитие

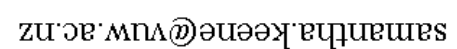
sets

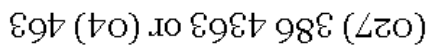
әиәәу вчдиеше

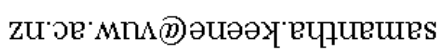
$8 \varepsilon t s$

\&9t (to) uo \&98t 988 ( $\angle z \circ)$ әиәәу вчдияие

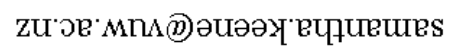

$8 \mathrm{stg}$

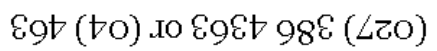
әиәәу вчдияие
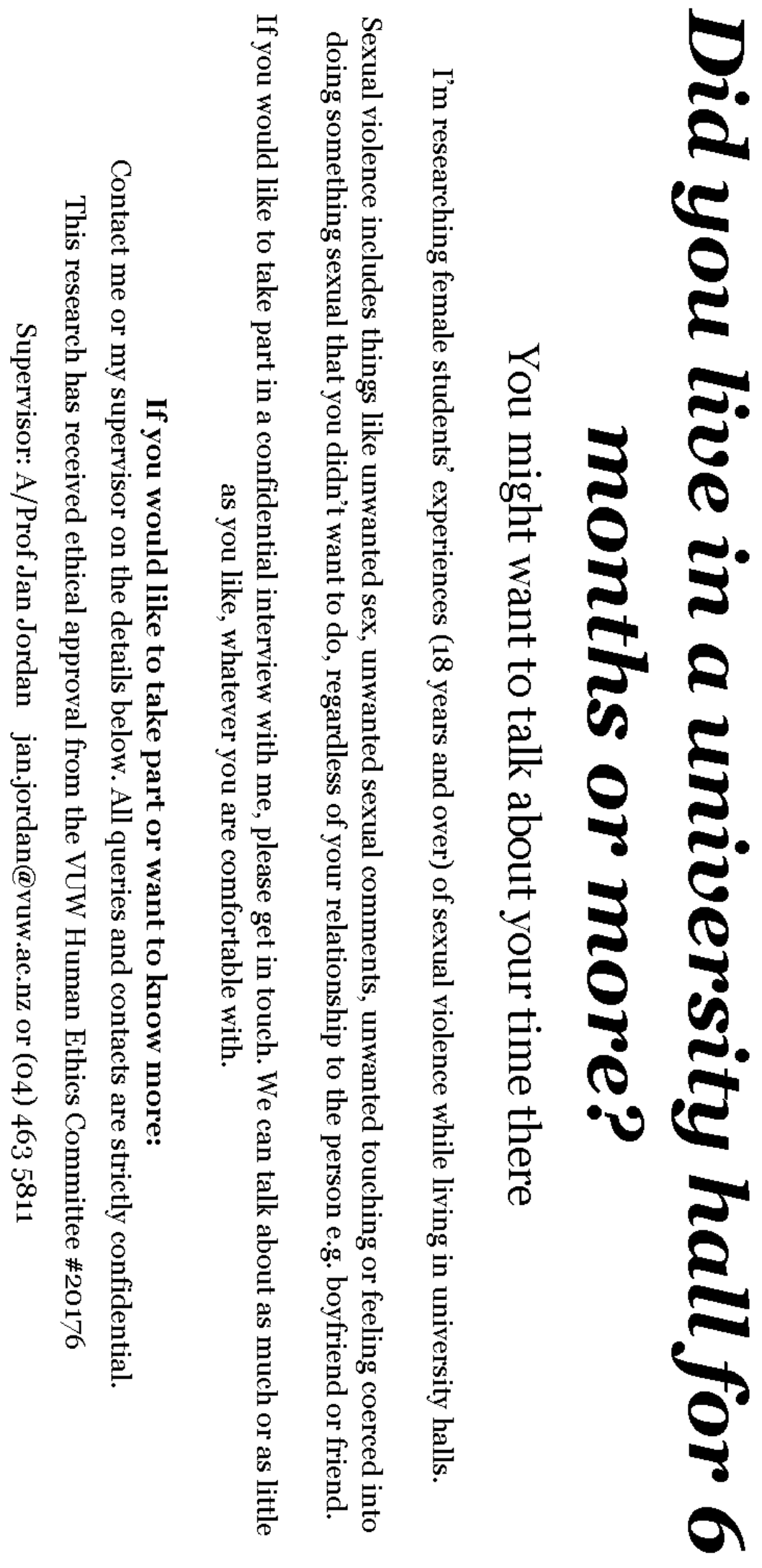


\section{Appendix C: Key Informant Information Sheet}

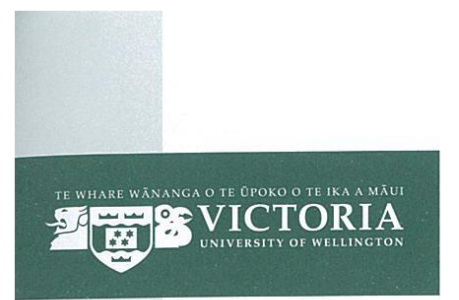

SCHOOL OF SOCIAL \& CULTURAL STUDIES

TE KURA MAHINGA TANGATA

VICTORIA UNIVERSITY OF WELLINGTON PO Box 600, Wellington 6140, New Zealand

Phone +64-4-4635317 Fax +64-4-4635064 Email sacs@vuw.ac.nz Website www.victoria.ac.nz/sacs

\section{Key Informant Information Sheet for a Study on Sexual Violence within University Halls}

\section{Researcher: Samantha Keene, School of Social and Cultural Studies, Victoria University of Wellington}

I am a Masters student in Criminology at Victoria University of Wellington. As part of this degree I am undertaking a research project leading to a thesis. The project I am undertaking is exploring the stories of young women living in university halls of residence and their experiences of sexual violence. I am also gaining insight from other stakeholders, such as those working in the sexual violence sector, about their understandings of sexual violence among university students living in university residences. This research project has received approval from the Victoria University Human Ethics Committee.

I am inviting people who work with university students and/or victim/survivors of sexual violence to take part in some key informant interviews. These interviews are to help me develop my research focus prior to interviewing participants.

Taking part in this research will involve taking part in a confidential interview with me that will take about an hour which will focus on your experiences working with student populations and your understanding of sexual violence among young female students. I will be asking questions such as:

- What is your experience working with student populations and/or victim/survivors of sexual violence?

- Do you think sexual violence among university populations is an issue?

- What would a good response to sexual violence look like by a university?

- How should universities be preventing sexual violence among students?

Participation in this interview is voluntary and you can withdraw from the study at any point prior to 30 June, 2014 without explanation. With your permission, these interviews will be tape recorded and later transcribed. Interview transcriptions will only be viewed by myself and my supervisor. All information provided in the interview will remain confidential and will only be used for the purposes of this research. Your name and all other identifying factors will not be used in this thesis.

All participants will be provided with a transcription of their interview which will be sent by email or in the post, whichever you prefer. This transcription can be amended or changed at any time up until 30 June, 2014. You can also request for the audio recording to be returned to you electronically at the completion of the project. Should any participants feel the need to withdraw from the research, they may do so without question at any time before 30 June, 2014. 


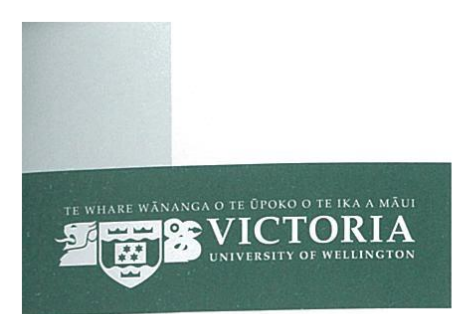

SCHOOL OF SOCIAL \& CULTURAL STUDIES TE KURA MAHINGA TANGATA

VICTORIA UNIVERSITY OF WELLINGTON PO Box 600, Wellington 6140, New Zealand

Phone +64-4-4635317 Fax +64-4-4635064 Email sacs@vuw.ac.nz Website www.victoria.ac.nz/sacs

The thesis will be submitted for marking to the School of Social and Cultural Studies and deposited in the library. A copy of the thesis will also be provided to the New Zealand Family Violence Clearinghouse library as part of a scholarship requirement. It is intended that one or more articles will be submitted for publication in scholarly journals and data may be presented at academic conferences. Transcriptions and recordings will be destroyed two years after the end of the project.

If you have any further questions or would like to receive further information about the project, please contact me at (027) 3864363 or samantha.keene@vuw.ac.nz or my supervisor, Jan Jordan, at the School of Social and Cultural Studies at Victoria University (04) 4635811 or jan.jordan@vuw.ac.nz.

Samantha Keene 


\section{Appendix D: Key Informant Consent Form}

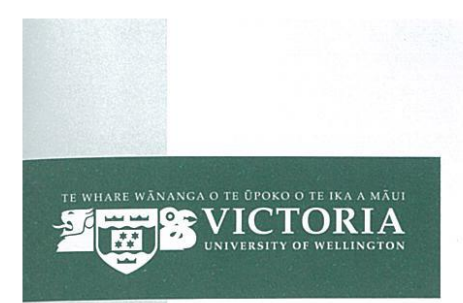

SCHOOL OF SOCIAL \& CULTURAL STUDIES

TE KURA MAHINGA TANGATA

VICTORIA UNIVERSITY OF WELLINGTON PO Box 600 , Wellington 6140 , New Zealand

Phone +64-4-4635317 Fax +64-4-4635064 Email sacs@vuw.ac.nz Website www.victoria.ac.nz/sacs

\section{KEY INFORMANT CONSENT FORM}

\section{Victoria University of Wellington}

\section{Consent to participation in research}

Title of project: Female students' experiences of sexual violence within university halls of residence

I have been given and have understood an explanation of this research project. I have been given the opportunity to ask questions and have them answered to my satisfaction. I understand that I am able to withdraw myself (or any information I have provided) from this project before 30 June, 2014, without having to give reasons.

I understand that any information I provide will remain confidential to the researcher and her supervisor Associate Professor Jan Jordan. I also understand that the published results will not use my name as an identifier, a pseudonym will be used, and no information attributed to me that will identify me will be published. I understand that the tape recording of my interview will be electronically wiped at the end of the project, unless I indicate that I would like the audio file to be returned to me.

- I agree to take part in this research.

- I understand that the data provided in this research will not be provided to any other person

- I understand that I will have an opportunity to check the interview transcript before publication and make amendments as I see fit.

- I understand that the data provided in this interview may be used for publication in academic journals and presentation at academic conferences.

I would like the tape recordings returned to me at the completion of the project.

I would like to receive a copy of this research when it is completed.

\section{Participant contact details for return of transcripts/recordings:}

Email address:

Postal address:

Signed:

Name of participant:

(Please print clearly)

Date: 


\section{Appendix E: Key Informant Interview Guide}

\section{Interview guide - Key informant interviews}

Introduction and informed consent:

- Introduce myself

- Introduce the project and explain why I am doing the research

- Ask if they have any questions about the research/interview

- Ensure that consent form has been completed and information sheet has been read

Demographic questions:

- What is your name?

- Could you tell me a bit about your role in the organisation?

- What sorts of services does the organisation provide?

- How long have you been working in this position?

- When did your interest in working with student populations begin?

- For Wellington Rape Crisis: When did you become interested in working in the sexual violence sector?

Questions/topics to be covered:

- Are many students using the services that the organisation provides?

- What sorts of services are they using?

- Why are they using them?

- How are they using them?

- Is sexual violence an area of focus within the organisation?

o Why is it?

- What specific services do you provide in this area?

- Are students using these services? In what way?

- What other support is available for students?

- Has sexual violence always been an area of focus?

- Why? Why not?

- Thinking about sexual violence, do you think it is a problem among university students?

- Why/why not?

- What types of things make students a particularly at-risk population?

- Many students, particularly first-year students, spend time living in a university hall of residence. Do you think sexual violence within university residences is an issue?

- How are universities currently responding to sexual violence?

- What about university residences?

- How do you feel about these responses?

- What would a good response to sexual violence look like by a university? - What about halls of residence?

- How should universities be preventing sexual violence among students?

Closing:

- Briefly touch on each of the main points that have been discussed in the interview and ask if there was anything else they would like to add or comments they would like to make 


\section{Appendix F: Participant Information Sheet}

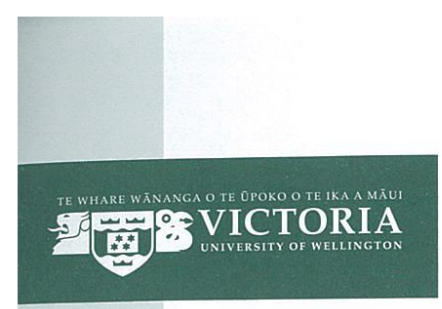

SCHOOL OF SOCIAL \& CULTURAL STUDIES

TE KURA MAHINGA TANGATA

VICTORIA UNIVERSITY OF WELLINGTON PO Box 600, Wellington 6140, New Zealand

Phone +64-4-4635317 Fax +64-4-4635064 Email sacs@vuw.ac.nz Website www.victoria.ac.nz/sacs

\section{Participant Information Sheet for a Study on Sexual Violence within University Halls}

\section{Researcher: Samantha Keene, School of Social and Cultural Studies, Victoria University of Wellington}

I am a Masters student in Criminology at Victoria University of Wellington. As part of this degree I am undertaking a research project leading to a thesis. The project I am undertaking is exploring the stories of women living in university halls of residence and their experiences of sexual violence. I am also gaining insight from other stakeholders, such as those working in the sexual violence sector, about their understandings of sexual violence among university students living in university residences. This research project has received approval from the Victoria University Human Ethics Committee.

I am inviting women who are aged 18 or over, who have lived in a university hall for six months or more in the past five years, and experienced sexual violence during their time in their hall, to participate in this study. For this research, sexual violence means an attempted, or completed, rape or sexual assault. This refers to any physical, sexual act that you did not consent to, were unable to consent to, felt forced or coerced into or was unwanted. Further, sexual violence also refers to unwanted sexual comments and unwanted touching, regardless of your relationship to the person e.g. boyfriend or friend.

Participation in this research is entirely voluntary. Participants will take part in a confidential interview with me that will take about an hour and, with your permission, will also be recorded. Participants have the right to request that recording of the interview be stopped during the interview. Interviews will take place in a safe, neutral location at a time that suits you.

This interview will ask questions such as:

- What were relations like between men and women in your hall?

- Could you tell me about safety in your hall? As in, did you feel safe?

- Could you tell me about a time that you experienced something sexual in the hall that you weren't happy about then, or are not happy about now?

- Did you seek support from an agency or support service such as Wellington Rape Crisis or Student Health Service?

All participants will be provided with a transcription of their interview which will be sent by email or in the post, whichever you prefer. This transcription can be amended or changed at any time up until 30 June, 2014. You can also request for the audio recording to be returned to you electronically at the completion of the project. Should 


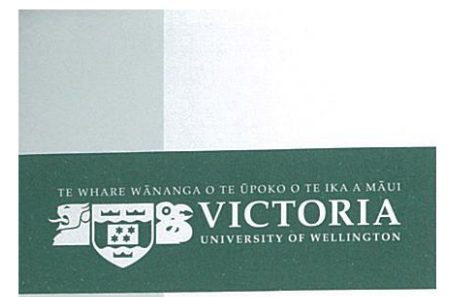

SCHOOL OF SOCIAL \& CULTURAL STUDIES TE KURA MAHINGA TANGATA

VICTORIA UNIVERSITY OF WELLINGTON PO Box 600, Wellington 6140, New Zealand

Phone +64-4-4635317 Fax +64-4-4635064 Email sacs@vuw.ac.nz Website www.victoria.ac.nz/sacs

any participants feel the need to withdraw from the research, they may do so without question at any time before 30 June, 2014.

The stories and experiences told during the interview will form the basis of my research project and will be put into a written report with your details remaining confidential. It will not be possible for you to be identified personally. A pseudonym will be assigned to your name to assure your confidentiality. Parts of the interview will be used in the thesis. All material collected will be kept confidential and no other person besides myself and my supervisor will see your complete interview transcripts.

The thesis will be submitted for making to the School of Social and Cultural Studies and deposited in the library. A copy of the thesis will also be provided to the New Zealand Family Violence Clearinghouse library as part of a scholarship requirement. It is intended that one or more articles will be submitted for publication in scholarly journals and data may be presented at academic conferences. Transcriptions and recordings will be destroyed two years after the end of the project.

If you have any further questions or would like to receive further information about the project, please contact me at (027) 3864363 or samantha.keene@vuw.ac.nz or my supervisor, Jan Jordan, at the School of Social and Cultural Studies at Victoria University (04) 4635811 or jan.jordan@vuw.ac.nz.

Samantha Keene 


\title{
Appendix G: Participant Consent Form
}

\author{
TE WHARE WANANGA TE OPOKO TE IIA A MIUU \\ SCHOOL OF SOCIAL \& CULTURAL STUDIES \\ TE KURA MAHINGA TANGATA \\ VICTORIA UNIVERSITY OF WELLINGTON PO Box 600, Wellington 6140, New Zealand \\ Phone +64-4-4635317 Fax +64-4-4635064 Email sacs@vuw.ac.nz website www.victoria.ac.nz/sacs \\ PARTICIPANT CONSENT FORM \\ Victoria University of Wellington \\ Consent to participation in research \\ Title of project: Female students' experiences of sexual violence within \\ university halls of residence \\ I have been given and have understood an explanation of this research project. I have \\ been given the opportunity to ask questions and have them answered to my \\ satisfaction. I understand that I am able to withdraw myself (or any information I \\ have provided) from this project before 30 June, 2014, without having to give \\ reasons. \\ I understand that any information I provide will remain confidential to the \\ researcher and her supervisor Associate Professor Jan Jordan. I also understand that \\ the published results will not use my name as an identifier, a pseudonym will be \\ used, and no information attributed to me that will identify me will be published. I \\ understand that the tape recording of my interview will be electronically wiped at the \\ end of the project, unless I indicate that I would like the audio file to be returned to \\ me. \\ - I agree to take part in this research. \\ - I understand that the data provided in this research will not be provided to any \\ other person \\ - I understand that I will have an opportunity to check the interview transcript \\ before publication and make amendments as I see fit. \\ - I understand that the data provided in this interview may be used for \\ publication in academic journals and presentation at academic conferences. \\ I would like the tape recordings returned to me at the completion of the \\ project. \\ I would like to receive a copy of this research when it is completed.

\section{Participant contact details for return of transcripts/recordings:} \\ Email address: \\ Postal address:
}

Signed:

Name of participant:

Date:

(Please print clearly) 


\section{Appendix H: Participant Interview Guide}

\section{Interview guide - Participants}

Introduction and informed consent:

- Introduce myself

- Explain the project and why I am doing the research

- Ask if they have any questions about the interview or the research

- Ensure that information sheet has been read and consent form signed

- Inform them that they can end the interview at any time, or turn off the recorder at any time

Demographic questions:

- What is your name?

- How old are you?

- Gender?

- Whereabouts do you live?

- What is your work-status?

- What is your ethnicity?

Interview questions:

- Could you tell me about the hall of residence that you lived in?

- What was management like in the hall?

- What sorts of activities were common in the hall?

- How were the relations between men and women in the hall?

- Could you tell me a bit about safety in the hall? As in, did you feel safe?

- Could you tell me about a time that you experienced something sexual in the hall that you weren't happy about then, or are not happy about now?

- How did it make you feel?

- Where/when did it happen? With whom?

- Were alcohol or drugs involved at all?

- How has it impacted you?

- Did you tell anyone [about the incident]?

- Yes - who did you tell?

- How did they respond?

- Did anything happen as a result of this?

- No - why didn't you tell anyone?

- Were there any other incidents such as this that happened?

- Did you seek support from an agency or support service such as Wellington Rape Crisis or Student Health Service?

- Were you aware of support services such as Wellington Rape Crisis and Student Health and Counselling?

- How do you think halls could make students safer?

Closing:

- Summarise the content of the interview, ask if there is anything that the participant would like to add or discuss. Further, ask if there are any comments that they would like to make. Thank them for their time and provide with a copy of contact details for support agencies to contact and koha for their time. 


\section{Appendix I: Participant Support Services Sheet}

\section{Resources and Contacts}

Sexual Abuse Support Agencies in the Wellington Area:

Tu Pakari Ora: Cuba Street Clinic

043859879

(SAAT - Sexual Assault Assessment and Treatment Service)

o80o 188881

Ground Level, 275 Cuba St

Te Aro, Wellington

Wellington Sexual Abuse HELP Foundation

$044997532(24 \mathrm{hr})$

(provides support and information, medical services,

info@wellingtonhelp.org.nz

counselling for children and adults, women and men,

individuals and families)

Level 2, James Smith Building

55 Cuba St

Wellington

www.wellingtonhelp.org.nz

Porirua Sexual Abuse HELP

$042378822(24 \mathrm{hr})$

(provides support and information, medical services, counselling for children and adults, women and men, individuals and families)

46 Mungavin Avenue

Porirua, Wellington

www.wellingtonhelp.org.nz

Wellington Rape Crisis

044735357

Level 3, Southmark House

203 Willis Street

Te Aro, Wellington

Opening hours: Monday - Friday 9.30am - 4.oopm

http://www.wellingtonrapecrisis.org.nz/

Support Services at Victoria University of Wellington:

VUW Student Health and Counselling Services

044635308 


\section{References}

Abbey, A. (2002). Alcohol-related sexual assault: A common problem among college students. Journal of Studies on Alcohol, 14, 118-128.

Abbey, A. A., Clinton-Sherrod, M., McAuslan, P., Zawacki, T., \& Buck, P. O. (2003). The relationship between the quantity of alcohol consumed and the severity of sexual assaults committed by college men. Journal of Interpersonal Violence, 18(7), 813-833.

Abbey, A., McAuslan, P., \& Ross, L. T. (1998). Sexual assault perpetration by college men: the role of alcohol, misperception of sexual intent, and sexual beliefs and experiences. Journal of Social and Clinical Psychology, 17, 167-195.

Adams-Curtis, L. E., \& Forbes, G. B. (2004). College women's experiences of sexual coercion: A review of cultural, perpetrator, victim and situational variables. Trauma, Violence and Abuse, 5, 91-113.

Allen, M. (2011). Violence and voice: using a feminist constructivist grounded theory to explore women's resistance to abuse. Qualitative Research, 11(1), 23-45.

Argiero, S. J., Dyrdahl, J. L., Fernandez, S. S., Whitney, L. E., Woodring, R. J. (2010). A cultural perspective for understanding how campus environments perpetuate rape-supportive culture. Journal of the Indiana University Student Personnel Association, 43, 26-40.

Banyard, V. L., Moynihan, M. M., Walsh W. A., Cohn, E. S., \& Ward, S. (2010). Friends of survivors: the community impact of unwanted sexual experiences. Journal of Interpersonal Violence, 25(2), 242-256.

Banyard, V. L., Ward, S., Cohn, E., Moorhead, C., \& Walsh, W. A. (2007). Unwanted sexual contact on campus: A comparison of women's and men's experiences. Violence and Victims, 22, 52-70. 
Basile, K. C., \& Smith, S. G. (2011). Sexual violence victimization of women: prevalence, characteristics, and the role of public health and prevention. American Journal of Lifestyle Medicine, 5(5), 407-417.

Bennett, P. (2013, February 22). Sexual violence sector deserves leadership. Retrieved from http://www.beehive.govt.nz/release/sexual-violencesector-deserves-leadership

Blain, G. (2014, October 3). SUNY will redefine sexual consent to address 'epidemic of sexual violence' on college campuses. Retrieved from http://www.nydailynews.com/new-york/suny-redefine-sexual-consentfight-epidemic-sexual-violence-colleges-article-1.1961762

Bogle, K. A. (2008). Hooking up: sex, dating and relationships on campus. New York: New York University Press.

Bohner, G., Friederike, E., Pina, A., Siebler, F., \& Tendayi Viki, G. (2009). Rape myth acceptance: cognitive, affective and behavioural effects of beliefs that blame the victim and exonerate the perpetrator. In M. Horvath \& J. Brown (Eds.), Rape: Challenging contemporary thinking (pp. 1745). Devon: Willan Publishing.

Borbridge, C. (1996). Incidence and type of sexual harassment in the University of Calgary residence halls (Masters thesis). University of Calgary, Alberta.

Boskey, E., Harper, J., \& Hilgenkamp, K. (2010). The truth about rape $\left(2^{\text {nd }}\right.$ edition). New York: Infobase Publishing.

Bourke, J. (2007). Rape: Sex, violence, history. Great Britain: Virago Press.

Boursnell, M., Lee, T., \& Chung, D. (2008). Tell us what you know: Surveying university students' attitudes about sexual assault. Melbourne: Australian Centre for the Study of Sexual Assault.

Boyd, C. (2011). The impacts of sexual assault on women. (ACSSA Resource Sheet). Melbourne: Australian Institute of Family Studies. Retrieved from http://www.aifs.gov.au/acssa/pubs/sheets/rs2/rs2.pdf 
Branch, K. A., Hayes-Smith, R., \& Richards, T. N. (2011). Professors' experiences with student disclosures of sexual assault and intimate partner violence: how "helping" students can inform teaching practices. Feminist Criminology, 6(1), 54-75.

Briere, J., \& Jordan. C. E. (2004). Outcome complexity and implications for assessment and treatment. Journal of Interpersonal Violence, 19(11), 1252-1276.

Brooks, A. (2007). Feminist standpoint epistemology: Building knowledge and empowerment through women's lived experience. In S. N. Hesse-Biber \& P. Leavy (Eds.), Feminist Research Practice: A Primer (pp. 53-82). Thousand Oaks: Sage Publications, Inc.

Brooks, A., \& Hesse-Biber, S. N. (2007). An invitation to feminist research. In S. N. Hesse-Biber \& P. Leavy (Eds.), Feminist Research Practice: A Primer (pp. 1 - 24). Thousand Oaks: Sage Publications, Inc.

Brown, A. L., Banyard, V. L., \& Moynihan, M. M. (2014). College students as helpful bystanders against sexual violence: gender, race and year in college moderate the impact of perceived peer norms. Psychology of Women Quarterly, 38(3), 350-362.

Brownmiller, S. (1975). Against our will: Men, women and rape. Harmondsworth: Penguin Books.

Bryant, A., \& Charmaz, K. (2007). The SAGE handbook of grounded theory (Eds.). London: Sage Publications Ltd.

Burgess, G. H. (2007). Assessment of rape-supportive attitudes and beliefs in college men: Development, reliability, and validity of the rape attitudes and beliefs scale. Journal of Interpersonal Violence, 22(8), 973-993.

Burt, M. R. (1991). Rape Myths. In M. E. Odem \& J.C. Warner (Eds.), Confronting rape and sexual assault (pp. 129-145). Lanham: S R Books. 
Cairns, K. V. (1994). A narrative study of qualitative data on sexual assault, coercion and harassment. Canadian Journal of Counselling, 28(3), 193205 .

Cairns, K. V., \& Wright, J. (1993). Survey of unwanted sexual attention in the University of Calgary residence complex. Canadian Association Against Sexual Harassment in Higher Education, 5, 105-117.

Calhoun, K. S., Mouilso, E. R., \& Edwards, K. M. (2012). Sexual assault among college students. In R. McAnulty (Ed.), Sex, love and psychology: sex in college, the things they don't write home about (pp. 299-324). Santa Barbara: ABC-CLIO.

Campbell, R. (2002). Emotionally involved: the impact of researching rape. Milton Park: Routledge.

Campbell, R., Dworkin, E., \& Cabral, G. (2009). An ecological model of the impact of sexual assault on women's mental health. Trauma, Violence \& Abuse, 10(3), 225-246.

Campbell, R., \& Raja, S. (1999). Secondary victimization of rape victims: insights from mental health professionals who treat survivors of violence. Violence \& Victims, 14, 261-275.

Campbell, R., \& Townsend, M. (2011). Defining the scope of sexual violence against women. In C. M. Renzetti, J. L. Edleson and R. K. Bergen (Eds.), Sourcebook on violence against women ( ${ }^{\text {nd }}$ ed) (pp. 95-110). Thousand Oaks: Sage Publications Ltd.

Campbell, R., Wasco, S. M., Ahrens, C. E., Sefl, T., \& Barnes, H. (2001). Preventing the "second rape": rape survivors' experiences with community service providers. Journal of Interpersonal Violence, 16(12), 1239-1259.

Cantalupo, N. C. (2014). Institution-specific victimization surveys: addressing legal and practical disincentives to gender-based violence reporting on college campuses. Trauma, Violence, \& Abuse, 15(3), 227-241. 
Carmody, M. (2009). Sex and ethics: The sexual ethics education programme for young people. South Yarra: Palgrave MacMillan.

Cashell-Smith, M. L., Connor, J. L., \& Kypri, K. (2007). Harmful effects of alcohol on sexual behaviour in a New Zealand university community. Drug and Alcohol Review, 26, 646-651.

Cermele, J. A. (2004). Teaching resistance to teach resistance: the use of selfdefense in teaching undergraduates about gender violence. Feminist Teacher, 15(1), 1-15.

Chappell, B. (2014, September 29). California enacts 'yes means yes' law, defining sexual consent. Retrieved from http://www.npr.org/blogs/thetwoway/2014/09/29/352482932/californ ia-enacts-yes-means-yes-law-defining-sexual-consent

Charmaz, K. (2006). Constructing grounded theory: A practical guide through qualitative analysis. London: Sage Publications Ltd.

Charmaz, K. (2014). Constructing grounded theory ( $2^{\text {nd }}$ ed). London: Sage Publications Ltd.

Clarkson, D. (2013, October 7). Regret led to rape complaint, says defence. The Press. Retrieved from http://www.stuff.co.nz/the-press/news/9253215/Regret-led-to-rapecomplaint-says-defence

Clarkson, D. (2014, February 19). Jailed for university hall rape. The Press. Retrieved from http://www.stuff.co.nz/the-press/news/9740041/Jailed-for-universityhall-rape

Clevenger, S. (2014). Criminal justice system treatment approaches for sexual assault victims. In T. N. Richards and C. D. Marcum (Eds.), Sexual victimisation: then and now (pp. 33-51). Thousand Oaks: Sage Publications, Inc. 
Clodfelter, T. A., Turner, M. G., Hartman, J. L., \& Kuhns, J. B. (2010). Sexual harassment victimization during emerging adulthood: a test of routine activities theory and a general theory of crime. Crime \& Delinquency, $56(3), 455-481$.

Connor, J., Gray, A., \& Kypri, K. (2010). Drinking history, current drinking and problematic sexual experiences among university students. Australian and New Zealand Journal of Public Health, 34(5), 487-494.

Connor, J., Psutka, R., Cousins, K., Gray, A., \& Kypri, K. (2013). Risky drinking, risky sex: a national study of New Zealand university students. Alcohol, Clinical and Experimental Research, 37(11), 1971-1978.

Cowlishaw, S. (2014, November 25). Sex victim reforms back on table. Retrieved from http://www.stuff.co.nz/national/politics/63498551/Sex-victimreforms-back-on-table

Crawford, E., O’Dougherty Wright, M., \& Birchmeier, Z. (2008). Drugfacilitated sexual assault: College women's risk perception and behavioral choices. Journal of American College Health, 57(3), 261-272.

Cue Davis, K. (2011). Sexual assault by strangers and non-intimate associates. In T. Bryant-Davis (Ed.), Surviving sexual violence: A guide to recovery and empowerment (pp. 37-47). Plymouth: Rowman and Littlefield Publishers.

Culbertson, K. A., Vik, P. W., \& Kooiman, B. (2001). The impact of sexual assault, sexual assault perpetrator type, and location of sexual assault on ratings of perceived safety. Violence Against Women, $7(8), 858-875$.

Daigle, L. E. (2012). Victimology: a text/reader. Thousand Oaks: Sage Publications Ltd.

Daly, K., \& Curtis-Fawley, S. (2006). Restorative justice for victims of sexual assault. In K. Heimer and C. Kruttschnitt (Eds.), Gender and crime: patterns of victimization and offending (pp. 230-268). New York: New York University Press. 
Dally, J., \& Clarkson, D. (2014, February 20). Women 'at risk of rape' in halls. The Press. Retrieved from

http://www.stuff.co.nz/the-press/news/9741829/Women-at-risk-ofrape-in-halls

Day, K. (1994). Conceptualising women's fear of sexual assault on campus: a review of causes and recommendations for change. Environment and Behavior, 26(6), 742-765.

DeGue, S. (2014). Preventing sexual violence on college campuses: Lessons from research and practice. Division of Violence Prevention, Centers for Disease Control and Prevention.

den Heijer, A. C. (2011). Managing the university campus: information to support real estate decisions. Delft: Eburon Academic Publishers.

Department of Prime Minister and Cabinet. (2014). Ministerial List. Retrieved from $\quad$ http://www.dpmc.govt.nz/cabinet/ministers/ministerial-list

Dunham, C. (2011). NZUSA campus safety audit: Are you OK? Results and recommendations. Wellington: NZUSA Federation Office.

Durrant, R., \& Thakker, J. Substance use and abuse: Cultural and historical perspectives. Thousand Oaks: Sage Publications, Ltd.

Edwards, K. M., Dardis, C. M., \& Gidycz, C. A. (2012). Women's disclosure of dating violence: a mixed methodological study. Feminism and Psychology, 22(4), 507-517.

Edwards, K. M., Dardis, C. M., Sylaska, K. M., \& Gidycz, C. A. (2014). Informal social reactions to college women's disclosure of intimate partner violence: associations with psychological and relational variables. 1-20.

Elder, V. (2013, June 20). Facebook shuts down student photo page. Otago Daily Times. Retrieved from http://www.odt.co.nz/campus/universityotago/261747/facebook-shuts-down-student-photo-page 
Else, A. (2012). 'Gender inequalities - Marriage, family and sex'. Te Ara - the Encyclopedia of New Zealand. Retrieved from http://www.TeAra.govt.nz/en/gender-inequalities/page-2

Estrich, S. (1987). Real rape: New perspectives on the law of rape. Cambridge: Harvard University Press.

Fanslow, J., \& Robinson, E. (2004). Violence against women in New Zealand: prevalence and health consequences. New Zealand Medical Journal, $117(1206)$, 1173-1184.

Fisher, B. S., Cullen, F. T., \& Turner, M. G. (2000). The sexual victimization of college women. Washington, DC: US Department of Justice, National Institute of Justice.

Fisher, B. S., Daigle, L. E., \& Cullen, F. T. (2009). Unsafe in the ivory tower: the sexual victimisation of college women. Thousand Oaks: Sage Publications Ltd.

Fisher, B. S., Karjane, H. M., Cullen, F. T., Blevins, K. R., Santana, S. A., \& Daigle, L. E. (2007). Reporting sexual assault and the Clery Act: situating findings from the National Campus Sexual Assault Policy Study within college women's experiences (pp. 65-83). In B. S. Fisher and J. J. Sloan III. Campus crime: legal, social, and policy perspectives (Eds.). Springfield, Illinois: Charles C Thomas.

Fisher, B. S., \& Wilkes, A. R. P. (2003). A tale of two ivory towers: a comparative analysis of victimization rates and risks between university students in the United States and England. Journal of Criminology, 43(3), 526-545.

Fitzgerald, N., \& Riley, J. K. (2000). Drug-facilitated rape: looking for the missing pieces. National Institute of Justice Journal, 243, 9-15.

Flack, W. F., Caron, M. L., Leinen, S. J., Breitenbach, K. G., Barber, A. M., Brown, E. N... Stein, H. C. (2008). "The Red Zone”: Temporal risk for unwanted sex among college students. Journal of Interpersonal Violence, 23(9), 1177-1196. 
Flores, R. L. (2010). Community interventions for adolescent victims of violence. In M. A. Paludi and F. Denmark (Eds.), Victims of sexual assault and abuse: resources and responses for individuals and families (pp. 169-178). California: ABC-CLIO, LLC.

Flowers, R. B. (2009). College crime: a statistical study of offenses on American campuses. North Carolina: McFarland \& Company, Inc.

Fontes, L. A., \& McCloskey, K. A. (2011). Cultural issues in violence against women. In C. M. Renzetti, J. L. Edleson \& R. K Bergen (Eds.), Sourcebook on violence against women ( ${ }^{\text {nd }}$ Ed) (pp. 151-168). Thousand Oaks: Sage Publications, Ltd.

Fouts, B., \& Knapp, J. (2001). A sexual assault education and risk reduction workshop for college freshmen. In A. J. Ottens and K. Hotelling (Eds.), Sexual violence on campus: Policies, programs and perspectives (pp. 98-119). New York: Springer Publishing Company, Inc.

Game, A., \& Metcalfe, A. (2003). The first year experience: Start, stay and succeed at uni. Sydney: The Federation Press.

Garcia-Moreno, C., Mitchell, K., \& Wellings, K. (2012). Sexual violence. In K. Wellings., K. Mitchell and M. Collumbien (Eds.), Sexual health: A public health perspective (pp. 47-58). Berkshire: Open University Press.

Gavey, N. (1991). Sexual victimization among Auckland University students: How much and who does it? New Zealand Journal of Psychology, 20, 63-70.

Gavey, N. (2005). Just sex? The cultural scaffolding of rape. East Sussex: Routledge.

Gengler-Dunn, D. (2007). A narrative inquiry of four female first-year, firstgeneration student perspectives of the university experience $(\mathrm{PhD}$ Thesis). Colorado State University, Colorado. 
Gidycz, C. A., \& Dardis, C. M. (2014). Feminist self-defense and resistance training for college students: a critical review of recommendations for the future. Trauma, Violence, \& Abuse, 15(4), 322-333.

Gidycz, C. A., Orchowski, L. M., \& Berkowitz, A. D. (2012). Preventing sexual aggression among college men: an evaluation of a social norms and bystander intervention program. Violence Against Women, 18, 264-288.

Glasby, J. (2011). Evidence, policy and practice: critical perspectives in health and social care (Ed.). Bristol: The Policy Press.

Glaser, B. G., \& Strauss, A. L. (2012). The discovery of grounded theory: strategies for qualitative research ( $7^{\text {th }}$ edition). The State University, New Jersey: Transaction Publishers.

Gousmett, N. (2013, November 26). Funding crisis for Rape Crisis. Retrieved from

http://www.stuff.co.nz/dominionpost/comment/9440456/Fundingcrisis- for-Rape-Crisis

Greeson, M. R., \& Campbell, R. (2013). Sexual assault response teams (SARTs): an empirical review of their effectiveness and challenges to successful implementation. Trauma, Violence, \& Abuse, 14(2), 83-95.

Greenfield, G. M., Keup, J. R., \& Gardner, J. N. (2013). Developing and sustaining successful first-year programmes: A guide for practitioners. San Francisco: John Wiley \& Sons, Inc.

Griffin, S. (1977). Rape: the all-American crime. In D. Chappell, R. Geis, and G. Geis (Eds.), Forcible rape: the crime, the victim, and the offender (pp. 47-66).

Grigsby, M. (2009). College life through the eyes of students. Albany: State University of New York. 
Gross, A. M., Winslett, A., Roberts, M., \& Gohm, C. L. (2006). An examination of sexual violence against college women. Violence Against Women, 12(3), 288-300.

Harnois, C. E. (2013). Feminist measures in survey research. Los Angeles: Sage Publications, Inc.

Hayes-Smith, R. M., \& Levett, L. M. (2010). Student perceptions of sexual assault resources and prevalence of rape myth attitudes. Feminist Criminology, 5(4), 335-354.

Hesse-Biber, S. N., \& Leavy, P. (2011). The practice of qualitative research $\left(2^{\text {nd }}\right.$ ed.). Los Angeles: Sage.

Huerta, M., Cortina, L. M., Pang, J. S., Torges, C. M., \& Magley, V. J. (2006). Sex and power in the academy: modelling sexual harassment in the lives of college women. Personality and Social Psychology Bulletin, 32(5), 616-628.

Hunt, T. (23 ${ }^{\text {rd }}$ April, 2014). University posts security guard on 'Rape Alley'. Retrieved from http://www.stuff.co.nz/national/crime/9967945/Universityposts- security-guard-on-Rape-Alley 23/04/2014

Jewkes, R., Sen, P., \& Garcia-Moreno, C. (2002). Sexual violence. In E. G. Krug, L. L. Dahlberg, J. A. Mercy, A. B. Zwi and R. Lozano (Eds.), World report on violence and health (pp. 147-174). Geneva: World Health Organisation.

Johnson, S. (2009). Therapist's guide to posttraumatic stress disorder intervention. London: Elsevier, Inc.

Johnson, H., Ollus, N., \& Nevala, S. (2007). Violence against women: an international perspective. New York: Springer.

Johnston, B. (2010). The first year at university: teaching students in transition. Berkshire: The Open University Press. 
Jordan, J. (2001). True "lies" and false "truths" (PhD Thesis). Victoria University of Wellington, New Zealand.

Jordan, J. (2004). Beyond belief?: Police, rape and women's credibility. Criminal Justice, 4(1), 29-59.

Jordan, J. (2008). Serial Survivors. Annandale: The Federation Press.

Jordan, J. (2011). Here we go round the review-go-round: rape investigation and prosecution - are things getting worse not better? Journal of Sexual Aggression: An international, interdisciplinary form for research, theory and practice, 17(3), 234-249.

Jordan, J. (2012). Silencing rape, silencing women. In J. M. Brown and S. L. Walklate (Eds.), Handbook on sexual violence (253-286). New York: Routledge.

Jordan, J. (2013). From victim to survivor - and from survivor to victim: reconceptualising the survivor journey. Sexual Abuse in Australia and New Zealand, 5(2), 48-56.

Jordan, C. E., Combs, J. L., \& Smith, G. T. (2014). An exploration of sexual victimization and academic performance among college women. Trauma, Violence and Abuse, 15(3), 191-200.

Julich, S., McGregor, K., Annan, J., Landon, F., McCarrison, D., \& McPhillips, K. (2011). Yes, there is another way! Canterbury Law Review, 17, 222228.

Kanin, E., \& Parcell, S. R. (1977). Sexual aggression: a second look at the offended female. Archives of Sexual Behaviour, 6(1), 67-76.

Kappler, K. E. (2011). Living with paradoxes: Victims of sexual violence and their conduct of everyday life. Germany: Springer Fachmedien.

Karjane, H. M., Fisher, B. S., \& Cullen, F. T. (2005). Sexual assault on campus: What colleges and universities are doing about it. Washington, DC: U. S. Department of Justice, Office of Justice Programs. 
Katz, J., May, P., Sorensen, S., \& DelTosta, J. (2010). Sexual revictimisation during women's first year of college: self-blame and sexual refusal assertiveness as possible mechanisms. Journal of Interpersonal Violence, 25, 2113-2126.

Kavanagh-Hall, E. (2012, October 25). Rape Crisis appalled by 'sick jokes'. Retrieved from http://www.stuff.co.nz/dominion-post/news/localpapers/the-wellingtonian/7860178/Rape-Crisis-appalled-by-sick-jokes

Kelly, L. (1988). Surviving sexual violence. Minneapolis: University of Minnesota Press.

Kelly, B. T., \& Torres, A. (2006). Campus safety: perceptions and experiences of women students. Journal of College Student Development, 47(1), 20-36.

Kilpatrick, D., \& Kanin, E. J. (1957). Male sexual aggression on a university campus. American Sociological Review, 22(1), 52-58.

Kilpatrick, D. G., Resnick, H. S., Ruggiero, K. J., Conoscenti, L. M., \& McCauley, J. (2007). Drug-facilitated, incapacitated and forcible rape: A national study. Washington, DC: U. S. Department of Justice, National Institute of Justice.

Knight, K. (2014, July 13). Tania Billingsley: No regrets about speaking out. Retrieved from http://www.stuff.co.nz/national/10261574/TaniaBillingsley-No-regrets-about-speaking-out

Koss, M. P., Gidycz, C. A., \& Wisniewski, N. (1987). The scope of rape: incidence and prevalence of sexual aggression and victimization in a national sample of higher education students. Journal of Clinical and Consulting Psychology, 55(2), 162-170.

Koss, M. P., \& Oros, C. (1982). The sexual experiences survey: A research instrument investigating sexual aggression and victimization. Journal of Consulting and Clinical Psychology, 50, 455-457. 
Koss, M. P., Wilgus, J. K., \& Williamsen, K. M. (2014). Campus sexual misconduct: restorative justice approaches to enhance compliance with Title IX guidance. Trauma, Violence, \& Abuse, 15(3), 242-257.

Krebs, C. P., Lindquist, C. H., Warner, T. D., Fisher, B. S., \& Martin, S. L. (2007). The Campus Sexual Assault (CSA) Study. Washington: Department of Justice, National Institute of Justice Programs.

Krebs, C. P., Lindquist, C. H., Warner, T. D., Fisher, B. S., \& Martin, S. L. (2009). The differential risk factors of physically forced and alcohol or other drug-enabled sexual assault among university women. Violence and Victims, 24(3), 302- 321.

Kypri, K., Paschall, M. J., Langley, J. D., Baxter, J., Cashell-Smith, M., \& Bordeaux, B. (2009). Drinking and alcohol-related harm among New Zealand university students: Findings from a national web-based survey. Alcoholism: Clinical \& Experimental Research, 33(2), 307-14.

Kypri, K., Paschall, M. J., Maclennan, B., \& Langley, J. (2007). Intoxication by drinking location: a web-based diary study in a New Zealand university community. Addictive Behaviors, 32(11), 2586-2596.

LaMonica, P., \& Pagliaro, E. M. (2013). Sexual assault intervention and the forensic examination. In R. M. Hammer., B. Moynihan., and E. M. Pagliaro (Eds.), Forensic nursing: a handbook for practice (pp. 349374). Burlington: Jones and Bartlett Publishing.

Langley, J. D., Kypri, K., \& Stephenson, S. C. R. (2003). Secondhand effects of alcohol use among university students: computerised survey. British Medical Journal, 327(7422). 1023-1024.

Lee, R. M. (1993). Doing research on sensitive topics. London: Sage Publications, Ltd.

Letherby, G. (2003). Feminist research in theory and practice. Buckingham: Open University Press. 
Liamputtong, P. (2007). Researching the vulnerable: a guide to sensitive research methods. London: Sage Publications Ltd.

Lichty, L. F., Campbell, R., \& Schuiteman, J. (2008). Developing a universitywide institutional response to sexual assault and relationship violence. Journal of Prevention \& Intervention in the Community, 36, (1-2), 5-22.

Lindquist, C. H., Barrick, K., Krebs, C., Croby, C. M., Lockard, A. J., \& SandersPhillips, K. (2013). The context and consequences of sexual assault among undergraduate women at Historically Black Colleges and Universities (HBCUs). Journal of Interpersonal Violence, 28(1), 2437$64)$.

Littleton, H. (2014). Interpersonal violence on college campuses: Understanding risk factors and working to find solutions. Trauma, Violence, \& Abuse, 15(4), 297-303.

Macy, R. J., Ermentrout, D. M., \& Johns, N. B. (2011). Health care for survivors of partner and sexual violence. In C. M. Renzetti, J. L. Edleson \& R. K Bergen (Eds.), Sourcebook on violence against women ( $2^{\text {nd }} \mathrm{Ed}$ ) (pp. 289308). Thousand Oaks: Sage Publications, Inc.

Mayhew, P., \& Reilly, J. (2007). The New Zealand Crime and Safety Survey: 2006. Wellington: Ministry of Justice.

Maynard, M. (1994). Methods, practice and epistemology: The debate about feminism and research. In M. Maynard and P. Purvis (Eds.), Researching women's lives from a feminist perspective. London: Taylor and Francis.

McMahon, S. (2010). Rape myth beliefs and bystander attitudes among incoming college students. Journal of American College Health, 59(1), 311.

McMahon, S., \& Banyard, V. L. (2012). When can I help? A conceptual framework for the prevention of sexual violence through bystander intervention. Trauma, Violence, \& Abuse, 13(1), 3-14. 
Mcnally, R. J. (2005). Remembering trauma. Cambridge: Harvard University Press.

Mikhailovich, K., \& Colbran, J. (1999). Changing the rules: Responding to sexual assault in an Australian University. Journal of Higher Education Police and Management, 21(1), 71-80.

Miller, S. L., Iovanni, L. \& Kelley, K. D. (2011). Violence against women and the criminal justice response. In C. M. Renzetti, J. L. Edleson \& R. K Bergen (Eds.), Sourcebook on violence against women ( $2^{\text {nd }} E d$ ) (pp. 267-289). Thousand Oaks: Sage Publications, Inc.

Millett, K. (2000). Sexual politics. Illinois: University of Illinois Press. (Original work published 1969).

Ministry of Women's Affairs (2009). Restoring Soul: Effective Interventions for adult victims/survivors of sexual violence. MWA: Wellington.

Mitchell, G. (2014, May 18). Let me go home. Salient. Retrieved from http://salient.org.nz/news/let-me-go-home

Moynihan, M. M., Banyard, V. L., Cares, A. C., Potter, S. J., Williams, M., \& Stapleton, J. G. (2014). Encouraging responses in sexual and relationship violence prevention: what program effects remain 1 year later? Journal of Interpersonal Violence, 1-23.

Nasta, A., Shah, B., Brahmanandam, S., Richman, K., Wittels, K., Allsworth, J., \& Boardman, L. (2005). Sexual victimization: Incidence, knowledge and resource use among a population of college women. Journal of Pediatric and Adolescent Gynecology, 18, 91-96.

National Union of Students. (2010). Hidden Marks: A study of women students' experiences of harassment, violence and sexual assault. London: National Union of Students.

Neidig, J. (2009). Sex, booze and clarity: defining sexual assault on a college campus. William \& Mary Journal of Women and the Law, 16(1), 179203. 
O’Neill, D. V. (2012). Responding to college campus acquaintance rape: contextual issues and the challenge of inter-organisational collaboration (PhD Thesis). University of Pennsylvania, Philadelphia.

Orchowski, L. M., \& Gidycz, C. A. (2012). To whom do college women confide following sexual assault? A prospective study of predictors of sexual assault disclosure and social reactions. Violence Against Women, 18(3), 264-288.

Ottens, A. J. (2001). (Ed.). Sexual violence on campus: Policies, programs and perspectives. New York: Springer Publishing Company.

Page, A. D. (201). True colors: police officers and rape myth acceptance. Feminist Criminology, 5(4), 315-334.

Paine, D. E. (2008). An exploration of three residence hall types and the academic and social integration of first year students (PhD thesis). University of South Florida, Florida.

Palmer, C., \& Parekh, V. (2014). Medical indicators and responses to intimate partner sexual violence. In P. Easteal and J. Y. Levy-Peck (Eds.), Intimate partner sexual violence: a multidisciplinary guide to improving services and support for survivors of rape and abuse (pp. 147-162). London: Jessica Kingsley Publishers.

Paul, L. A., Walsh, K., McCauley, J. L., Ruggiero, K. J., Resnick, H. S., \& Kilpatrick, D. G. (2013). College women's experiences with rape disclosure: A national study. Violence Against Women, 19(4), 486-502.

Payne, R. (2008). Challenges responding to sexual violence: Differences between campuses and communities. Journal of Criminal Justice, 36, 224-230.

Pearson, A. (2003). Cultural messages contribute to the prevalence of date rape. In J. Haley (Ed.), Date rape (62-71). Missouri: Greenhaven Press.

Petrak, J., \& Hedge, B. (2002). The trauma of sexual assault: treatment, prevention and practice (Eds.). West Sussex: John Wiley \& Sons, Ltd. 
Phipps, A., \& Smith, G. (2012). Violence against women students in the UK: time to take action. Gender and Education, 24(4), 357-373.

Prebble, T., Hargraves, H., Leach, L., Naidoo, K., Suddaby, G., \& Zepke, N. (2005). Impact of student support services on student outcomes in undergraduate tertiary study. Wellington: Ministry of Education.

Quinlan, E., Clarke, A., \& Horsley, J. (2010). From outrage to action: countering the institutional response to sexualized violence on university campuses. Canadian Woman Studies, 28(1), 46-55.

Radio New Zealand. (2014, July 5). Christchurch loses rape crisis centre. Retrieved from

www.radionz.co.nz/news/regional/248961/christchurch-loses-rapecrisis-centre

Renn, K. A. (2014). Women's colleges and universities in a global context. Baltimore: John Hopkins University Press.

Rothman, E., \& Silverman, J. (2007). The effect of a college sexual assault prevention program on first-year students victimisation rates. Journal of American College Health, 55(5), 283-290.

Russell, D. E. (1975). The politics of rape: the victim's perspective. New York: Stein and Day.

Sabina, C., \& Ho, L. Y. (2014). Campus and college victim responses to sexual assault and dating violence: disclosure, service utilization, and service provision. Trauma, Violence, \& Abuse, 15(3), 201-226.

Sampson, R. (2002). Acquaintance rape of college students (problem-oriented guides for Police series, No 17). United States Department of Justice, Office of Community Oriented Policing Services.

Sanday, P. R. (2007). Fraternity gang rape: sex, brotherhood and privilege on campus ( $2^{\text {nd }}$ Ed). New York: New York University. 
Schwartz, M. D., \& DeKeseredy, W. S. (1997). Sexual assault on the college campus: the role of male peer support. Thousand Oaks: Sage Publications, Ltd.

Schwartz, M. D., \& Leggett, M. S. (1999). Bad dates or emotional trauma? The aftermath of campus sexual assault. Violence Against Women, 5, 251271.

Scott, D.J. (2009). A closer look at completion in higher education in New Zealand. Journal of Higher Education Policy and Management, 31, 101108.

Shadwell, T. (2014a, August 8). Alleged sexual assault by National War Memorial. Dominion Post. Retrieved from http://www.stuff.co.nz/dominion-post/news/10363764/Allegedsexual-assault-by-National-War-Memorial

Shadwell, T. (2014b, August 13). Uni's rape advice: wear runners, carry whistle. Retrieved from http://www.stuff.co.nz/life-style/life/10376703/Unisrape-advice-wear-runners-carry-whistle 13/o8/2014

Sloan, J. J., \& Fisher, B. S. (2011). The dark side of the ivory tower: Campus crime as a social problem. New York: Cambridge University Press.

Smith, C. P., \& Freyd, J. J. (2013). Dangerous safe havens: institutional betrayal exacerbates sexual trauma. Journal of Traumatic Stress, 26, 119-124.

Smith, I. (2013). Prevention, response, and referral pathways for cases of intimate partner violence and sexual violence at a New Zealand university: A preliminary assessment (Honours Thesis). University of Auckland, New Zealand.

Smith, P. M. (2012). A concise history of New Zealand (2 ${ }^{\text {nd }}$ Ed). Port Melbourne: Cambridge University Press.

Sochting, I., Fairbrother, N., \& Koch, W. J. (2004). Sexual assault of women: Prevention efforts and risk factors. Violence against women, 1O(1), 7393. 
Steward, I., \& Dennett, K. (29 October, 2014). Roast Busters case: no charges to be laid. $\quad$ Retrieved from http://www.stuff.co.nz/national/crime/10674764/Roast-Busters-caseNo-charges-to-be-laid

Strauss, A., \& Corbin, J. M. (1997). Grounded theory in practice (Eds.). Thousand Oaks: Sage Publications Ltd.

Suarez, E., \& Gadalla, T. M. (2010). Stop blaming the victim: a meta-analysis on rape myths. Journal of Interpersonal Violence, 25(11), 2010-2035.

Temkin, J., \& Krahe, B. (2008). Sexual assault and the justice gap: a question of attitude. Oxford: Hart Publishing.

Tinsley, Y., \& McDonald, E. (2011). Is there any other way? Possible alternatives to the current criminal justice process. Canterbury Law Review, 17, 192 $-221$.

Towl, A. (2004). Alcohol use and tertiary students in Aotearoa-New Zealand. Wellington: ALAC.

Ullman, S. (2010). Talking about sexual assault: society's response to survivors. Washington, DC: American Psychological Association.

Urquhart, C. (2013). Grounded theory for qualitative research: a practical guide. London: Sage Publications Ltd.

van der Meer, J., Scott, S., \& Neha, T. (2010). Retention of first-year Maori students at university. Mai Review, 2, 1-14.

Victoria University of Wellington. (2014). Marsden funding success for Victoria researchers. Retrieved from

https://www.victoria.ac.nz/news/2014/marsden-funding-success-forvictoria-researchers 04/11/2014

Vladutiu, C. J., Martin, S. L., \& Macy, R. J. (2011). College-or University-based sexual assault prevention programs: a review of program outcomes, 
characteristics, and recommendations. Trauma, Violence, \& Abuse, 12(2), 67-86.

Walker, H. (2010). "All work and no play?" The transition to university (Masters thesis). University of Otago, New Zealand.

Walsh, W. A., Banyard, V. L., Moynihan, M. M., Ward, S., \& Cohn, S. E. (2010). Disclosure and service use on a college campus after an unwanted sexual experience. Journal of Trauma and Dissociation, 11(2), 134-151.

Wasserman, C. (2003). Dating violence on campus: a fact of life. Washington: National Center for Victims of Crime.

Weekes, J. (2014, October 8). Facebook groups the 'ugly underbelly of sexism'. Retrieved from

http://www.nzherald.co.nz/nz/news/article.cfm?c id=1\&objectid=11339 350

Whitehouse Taskforce for Protect Students from Sexual Assault. (2014). Not alone: The first report of the White House Task Force to Protect Students from Sexual Assault. Washington: White House.

Wolitzky-Taylor, K. B., Resnick, H. S., Amstadter, A. B., McCauley, J. L., Ruggiero, K. J., \& Kilpatrick, D. G. (2011). Reporting rape in a national sample of college women. Journal of American College Health, 59(7), $582-587$.

Wood, J. (2009). Feminist standpoint theory. In S. Littlejohn and K. Foss (Eds.), Encyclopedia of communication theory (pp. 397-399). Thousand Oaks: Sage Publications, Inc.

Wright, L. (2012). Reinventing the squeal: young New Zealand women negotiating space in the current sexual culture (PhD Thesis). Victoria University of Wellington, New Zealand.

Wykes, N., \& Welsh, K. (2009). Violence, gender and justice. Thousand Oaks: Sage Publications Ltd. 
Yuan, N. P., Koss, M. P., \& Stone, M. (2006). The psychological consequences of sexualtrauma. Harrisburg, PA: VAWnet, a project of the National Resource Center on Domestic Violence. Retrieved 29 September, 2014 from: http://www.vawnet.org

Zeller, W. J. (2005). First-year student living environments. In M. L. Upcraft, J. N. Gardner \& B. O. Barefoot (Eds.), Challenging and supporting the first-year student: a handbook for improving the first year of college (pp. 410-427). San Francisco: John Wiley \& Sons, Inc.

Zepke, N., Leach, L., Prebble, T., Campbell, A., Coltman, D., Dewart, B... Wilson, S. (2005). Improving tertiary student outcomes in the first year of study. Wellington: Teaching and Learning Research Initiative. Retrieved from

http://www.tlri.org.nz/sites/default/files/projects/9209 finalreport.pdf

Zinzow, H. M., \& Thompson, M. (2011). Barriers to reporting sexual victimization: prevalence and correlates among undergraduate women. Journal of Aggression, Maltreatment \& Trauma, 20, 711-725. 